Distribu

\title{
MICROMODEL FOAM FLOW STUDY
}

By

K. T. Chambers

C. J. Radke

October 1990

Work Performed Under Contract No. AC03-76SF00098

Prepared for

U.S. Department of Energy

Assistant Secretary for Fossil Energy

Thomas B. Reid, Project Manager

Bartlesville Project Office

P.O. Box 1398

Bartlesville, OK 74005

Prepared by

University of California

Chemical Engineering Department

Berkeley, CA 94720

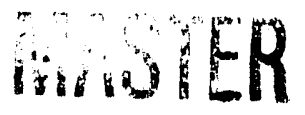




\section{Table of Contents}

List of Tables . . . . . . . . . . . . . . . . v

List of Figures . . . . . . . . . . . . . . . . v

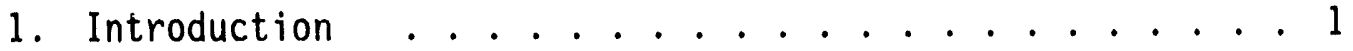

2. Micromodel Fabrication ............ 2

3. Micromodel Specifications ........... . 5

4. Foam Flow Apparatus . . . . . . . . . . . 8

5. Experimental Procedure ............ 12

6. Chemicals ............... . . 13

7. Capillary Phenomena in Micromodels ....... 13

1. Overview ................ 13

2. Application of Percolation Concapts to Micromodels 15

3. Fxtrapolating 2-D Micromodel Observations to 3-D Behavior ............. 19

8. Importance of Pore Structure on Capillary Instabilities 25

9. Lamellae Generation Mechanisms ......... 31

1. Snap-off .............. . 31

2. Weeping-Flow Breakup .......... 46

3. Lamella Division" ............. 56

4. Lamella Leave-Behind . . . . . . . . . . 62

10. Coalescence Mechanisms . . . . . . . . . 67

1. Capilitary-Pressure Considerations . . . . . 67

2. Dynamic Capillary-Pressure-Induced Coàlescence . . 72

3. Lamellae Coalescence by Gas Diffission ...... 82

11. Summary . . . . . . . . . . . . . 86

Nomenclature . . . . . . . . . . . . . . . . . 89

References ................. 92

Appendix A: Camera Assembly ............ . 97

Appendix B: Characteristic Time for Gas Diffusion in

Bulk Foam . . . . . . . . . . . 99 


\section{List of Tables}

Table 1: Micromodel specifications ...........7

\section{List of Figures}

Figure 1: Schematic of micromode1 ........... 4

Figure 2: Photograph of micromodel. Scale provides a guage of size ............ 6

Figure 3: Experimental setup for micromodel only ..... 9

Figure 4: Voltage-dividing circuit (from Maniowe [19] ... 11

Figure 5: Schematic comparing (a) regular lattices to

(b) Bethe trees. Both figures represent a coordination number of 4 ......... 16

Figure 6: Cross-sectional profile of an ethed pore throat

(from Wardlaw et al. [37]) . . . . . . 22 
Figure : 7: Effective diameter of a pore etched in a glass plate versus its width (from Wardlaw et al. [37]). The different symbols correspond to data for four different micromorlels.

Figure 8: Depth of a pore etched in a glass plate versus its width (from Wardlaw et al. [37]). The different symbo's correspond to data for four different micromodels...

Figure 9: Schematic of capillary-driven flow induced by an infinitesimal disturbance of wavelength $\lambda$ in a cylindrical capillary containing an inviscid nonwetting thread. Higher curvature at 1 compared to 2 leads to a capillarypressure gradient that drives liquid toward 1 resulting in the formation of a liquid collar.

Figure 10: Microphotographs of Wood's metal (light shading) occupying (a) $73 \%$ and (b) $90 \%$ of the pore volume in Berea sandstone (from Wardlaw et al [45]). The rock (dark shading) was removed by dissolution in acid..

Figure 11: Stability of nonwetting threads in noncircular capillaries showing (a) stable and (b) unstable configurations resulting from a local saturation disturbance (dotted line). The initial and perturbed principal radii of curvature are $R_{1}$ and $R_{2}$, respectively

Figure 12: Schernatic of snap-off in a constricted, noncircular capillary: (a) gas enters pore constriction, (b) gas front expands into downstream nore body setting up a driving force for liquid flow, (c) gas bubble snaps-off leaving a liquid lens, (d) a lamella forms as gas reinvades the pore constriction.

Figure 13: Schematic of bubble-deposited liquid profiles in (a) cylindrical and (b) square-rross-sectional capillaries. The values of 1 and $1 / 2$ correspond to the normalized cuivatures for cylindrical and square cross sections, respectively.........

Figure 14: Microphotographs of snap-off recorded during gas (air) injectior, into an etched glass micromodel, which was originally saturated with surfactant solution, at a superficial gas velocity of $13 \mathrm{~m} / \mathrm{day}$ : (a) gas enters pore throat highlighted with an arrow, (b) gas expands into downstream liquid-filled pore body, (c) formation of liquid collar in the pore throat, (d) snap-off occurs less than $1 / 30$ of a second after the collar appears in (c).

Figure 15: Microphotographs of snap-off repeating itself in the same location and at the same flow cciditions discussed in Figure 14: (a) gas enters pore throat, (b) gas expands into downstream pore body, (c) gas bubble snaps-off........

Figure 16: Microphotographs showing the deactivation of the snap-off site discuss $\mathrm{d}$ in Figures 14 and 15 due to an increase in the local gas saturation and capillary pressure: (a) gas enters pore throat, (b) curvature at gas front is modified (increased) by increased gas saruration in downstream pore body, (c) continued increase in curvature at bubble front, (d) gas completely fills pore body without snapping-off.... 
Figure 17: Microphotographs of an intermittent snap-off event recorded while injecting an $80 \%$ quality foam comprised of air and surfactant solution at a total superficial velocity of $62 \mathrm{~m} /$ day: (a) liquid (surfactant solution) occupied pore throat highlighted with an arrow, (b) gas enters throat form left and displaces liquid, (c) liquid imbibes into pore throat 4 seconds later and snaps-off a bubble as a result of a local capillary-pressure fluctuation

Figure 18: Microphotographs of weeping flow causing snap-off upstream of a pore throat recorded while injecting wetting liquid (surfactant solution) at a superficial velocity of $13 \mathrm{~m} / \mathrm{d}$.ly following a foam flood: (a) liquid flowing from top to bottom forces a gas bubble (highlighted with an arrow) into a pore throat, (b) microphotograph recorded $1 / 30$ of a second later indicates that a bubble snapped-off at or slightly upstream of the minimum constriction in the throat (the dim outline running the length of the pore throat and into the large pore body located just above the date and time information is a consequence of the high bubble velocity and limited time resolution of the video equipment (1/30 of a second)), (c) repeated snap-off due to weeping flow in the same pore throat resulting in the formation of bubbles with radii on the order of the pore-throat size....................

Figure 19: Microphotographs of weeping-flow breakup of a long bubble in a pore constriction at the same conditions as Figure 18: (a) long bubble (highlighted with an arrow) is trapped against a pore constriction, (b) wetting-liquid flow forces bubble into constriction, (c) gas breaks away from pore wall in constriction indicating the formation of an unstable liquid collar, (d) breakup occurs less than $1 / 30$ of a second later....

Figure 20: Microphotographs of lamella division at a $Y$-shaped pore junction during gas (air) injection at a superficial velocity of $13 \mathrm{~m} /$ day into an etched glass micromodel initially saturated with surfactant solution: (a) lamella (highlighted with an arrow) flows toward $Y$-shaped junction, (b) lamella bows away from pore walls as it expands into jurction, (c) lamella continues to bow as it expands farther into junction (note apparent contact angle of $70^{\circ}$ with pore walls), (d) lamella divides upon contact with iar pore wall.

Figure 21: Microphotographs of intermittent lamella division at the same conditions as Figure 2.20: (a) lowe: flow branch leading from pore body at left center of microphotograph contains f Jam that flows from left to right while upper branch contains trapped foam, (b) foam is mobilized in upper branch, (c) large foam bubble with a lamella spanning the entire pore body convects toward outlet of body, (d) lamella divides at outlet of pore body...

Figure 22: Microphotographs illustrating that small foam bubbles do not readily divide: (a) foam flows in both upper and lower branches through the same site and at the same conditions discussed in Figure 21, (b) small foam bubbles separate at the downstream side of the pore body and flow out of it without dividing, (c) more small bubbles flowing without dividing but note that a large gas bubble enters from the left, (d) the large bubble divides and the newly created lamella is highlighted with an arrow. 
Figure 23: Microphotographs of lamella leave-behind recorded while injecting an $80 \%$ quality foam comprised of air and surfactant solution at a total superficial velocity of $31 \mathrm{~m} /$ day: (a) two gas fingers in the upper right converge on the same surfactant-saturated region, (b) slower gas finger still converging, (c) gas converges to form a lens, (d) liquid has drained out of lens leaving a lamella that opens up into Plateau borders (note that the area of interest has shifted to the center

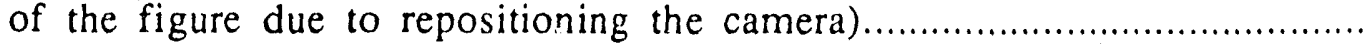

Figure 24: Schematic of a typical disjoining pressure curve. The distances of $\mathrm{h}$ are of order 10 to $100 \mathrm{~nm}$, while the values of $\Pi$ are near $10 \mathrm{kPa}$.

Figure 25: Schematic of a foam lamella................................ 70

Figure 26: Microphotographs of dynamic, capillary-pressure-induced lamella coalescence recorded while injecting an $80 \%$ quality foam comprised if air and surfaciant solution at a total superficial velocity of $45 \mathrm{~m} /$ day: (a) lamella (highlighted with an arrow) convects from right to left into a pore body, (b) lamella stretches as it expands into the pore body because it must conform to the pore walls, (c) lamella coalesces at the maximum radius of the pore body, (d) no nearby lamellae break in response to the coalescence event in (c).............................

Figure 27: Microphotograph showing repeatable coalescence at the same site and at the same conditions as Figure 2.26: (a) and (b) lamella conforms to pore walls as it convects through a pore body, (c) lamella coalesces without inducing additional coalescence.

Figure 28: Predictions for critical capillary pressure $\widetilde{P}_{c}$ " as a function of the pore body to pore-throat aspect ratio, $R_{b} / R_{l}$, and modified capillary number $C a_{m}$ ( $\because$ om Jimenez and Radke [63]). $\mathrm{Ca}_{m}$ is approximately $10^{5}$ times $\mathrm{Ca}$ for the same gas velocity, liquid viscosity, and surface tension.

Figure 29: Comparison of experimental and theoretical results for the critical capillary pressure $\widetilde{\mathrm{P}}_{\mathrm{c}}{ }^{\text {" }}$ as a function of the modified capillary number Ca' for an assumed $R_{b} / R_{l}$ of 1.45 for foam coalescence in an $81-\mu \mathrm{m}^{2}$ sandpack (from Jimenez and Radke [63])

Figure 30: Microphotographs of lamella coalescence in bulk foam due to gas diffusion: (a) two small nearly :pherical bubbles (highlighted with an arrow') in contact with larger foam bubbles, (b) bubbles shrink due to gas diffusion into larger bubbles, (c) small bubbles continue to shrink at an accelerating pice, (d) a lamella coalesces due to the disappearance of the small bubbles....

Figure 31: Schematic of gas diffusion without coalescence in lamellae foam: (a) gas diffuses from left to right across a lamella located at point 1, (b) a stable configuration results without any further driving force for gas diffusion 


\section{Micromodel Foam Flow Study}

\section{Introduction}

The bubble-size distribution or texture is set during foam flow through por'sus media by the balance between lamellae generation and destruction mechanisms. The texture has a profound effect on the flow behavior [1-11]. Unfortunately, the corventional approach toward understanding fluid flow in porovs media through core-flood experiments sheds no light on the pore-level events that shape foam texture. Porous-media micromodels, on the other hand, consisting of of transparent glass plates which have been chemically etched to represent the flow networks existing in natural porous media, do reveal the pore-level capillary instabilities responsible for shaping the foam texture [12-18]. Furthermore, micronodels illustrate the manner by which foam transports through porous media.

This report presents the results of a mic omodel foam flow study. It became apparent during the course of this study that foam flow behavior in the micrornodels is sensitive to small variations in the gas and surfactant delivery rates due to the very small pore volumes of the models (less than $0.2 \mathrm{~cm}^{3}$ ). The two-dimensionality of the micromodels also limits the applicability of the flow results to describe flow behavior in naturally-occurring porous media. Although several other investigators have used micromodel flow results in a quantitative manner $[12,16,17,19]$, precise measurements of the pressure-drop-versus-flow-rate behavior are not used to quantify foam flow in this micromodel study as a result of the limitations presented above. Instead observations of pore-level phenomena in the micromodels during foam floods are used to develop rate expressions describing lamellae generation and coalescence. The developed rate expressions are then incorporated into a mathematical model to predict foam texture within 
the framework of the population balance on the number density of foam bubbles [1-3, 7 , 11].

The geometry and wettability of the pore structure exert a strong influence on capillary phenomena in porous media. Consequently, a description of the micromodels used in this study is required before presenting the results on capillary phenomena and their role in shaping foam texture and flow behavior.

\section{Micromodel Fabrication}

The process of fabricating a transparent, porous-medium micromodel consists of several steps. The micromodels used in this study were created by Adobe Labs, P.O. Box 1351, Socorro, NM 87801. The first step in the process was to obtain a two-dimensional transparency of the network pattern chosen to represent the pore structure. Both regular network patterns [12-16] and patterns copied from thin-sections of reservoir rock $[12,17$, 19] have been used as the basis for micromodel transparencies. The latter approach was employed in the fabrication of the micromodels used in this study. The transparency for these micromodels was obtained irom a photograph of an epoxy-impregnated thin-section of Kuparuk sandstone, which was recovered from the Prudhoe Bay oil field in Alaska [20]. The thin-section was prepared by first impregnating a core of Kuparuk sandstone with a dyed epoxy resin. After the resin had solidified, a very thin slice was cut from the core and then mounted between two microscope slides. A photograph of the thin-section was then magnified by roughly a factor of five and transferred onto a transparency. The pore space shows up as black on the transparency while the rock matrix appears clear. Injection and production ports and flow-distribution channels were then drawn in black at opposite ends of the rectangular transparency.

The next step in the process was to transfer the image on the transparency onto a piece of glass. This was accomplished by first treating a glass plate with KMER resin. The treated glass plate was then overlayed with the transparency and subjected to UV light 
for a period of a few minutes. After this step was completed, KMER developer was applied to the glass plate. The undeveloped resin was then washed off of the plate, leaving the porous-medium pattern and the fluid injection and withdrawl ports. After protecting the glass areas outside of the flow network pattern, including the back and sides, with wax or lightly adhering waterproof tape, the plate was immersed in a bath of concentrated hydrofluoric acid. The glass plate was left in the acid bath for roughly ten minutes in order to obtain approximately $50 \mu \mathrm{m}$-deep, etched flow channels. The procedure outlined above was then repeated on a second glass plate where the original transparency had been turned over to produce a mirror image of the flow network. Next, injection and production ports were drilled into one of the glass plates. Finally, the flow networks in the two glass plates were aligned under a microscope, and then the plates were fused together in a $720^{\circ} \mathrm{C}$, muffle-oven for 20 minutes.

Figure 1 provides a schematic for the two, nominally identical micromodels used in this study. The cross-hatched shading indicates that part of the model actually etched with the Kuparuk saridstone pattern. Both of the micromodels used in this study were fabricated from the sime transparency and in the same manner. However, due to nonhomogeneities in the glass plates and slight variations in exposure and etching times, the flow network in the second micromodel was not as well-connected as that existing in the first. This was not a critical limitation in this study, as pointed out in the introduction to this chapter, because the micromodels were used primarily to gain qualitative insights into capillary events occurring during foam flow. Figure 1 illustrates the etched inlet and outlet ports, as mentioned earlier, for introducing and extracting fluids. Note also the 150 $\mu \mathrm{m}$-wide etched channels at both ends of the models that are normal to the flow direction. These channels were included to ensure an even lateral distribution of fluids throughout the models during the flow experiments. 


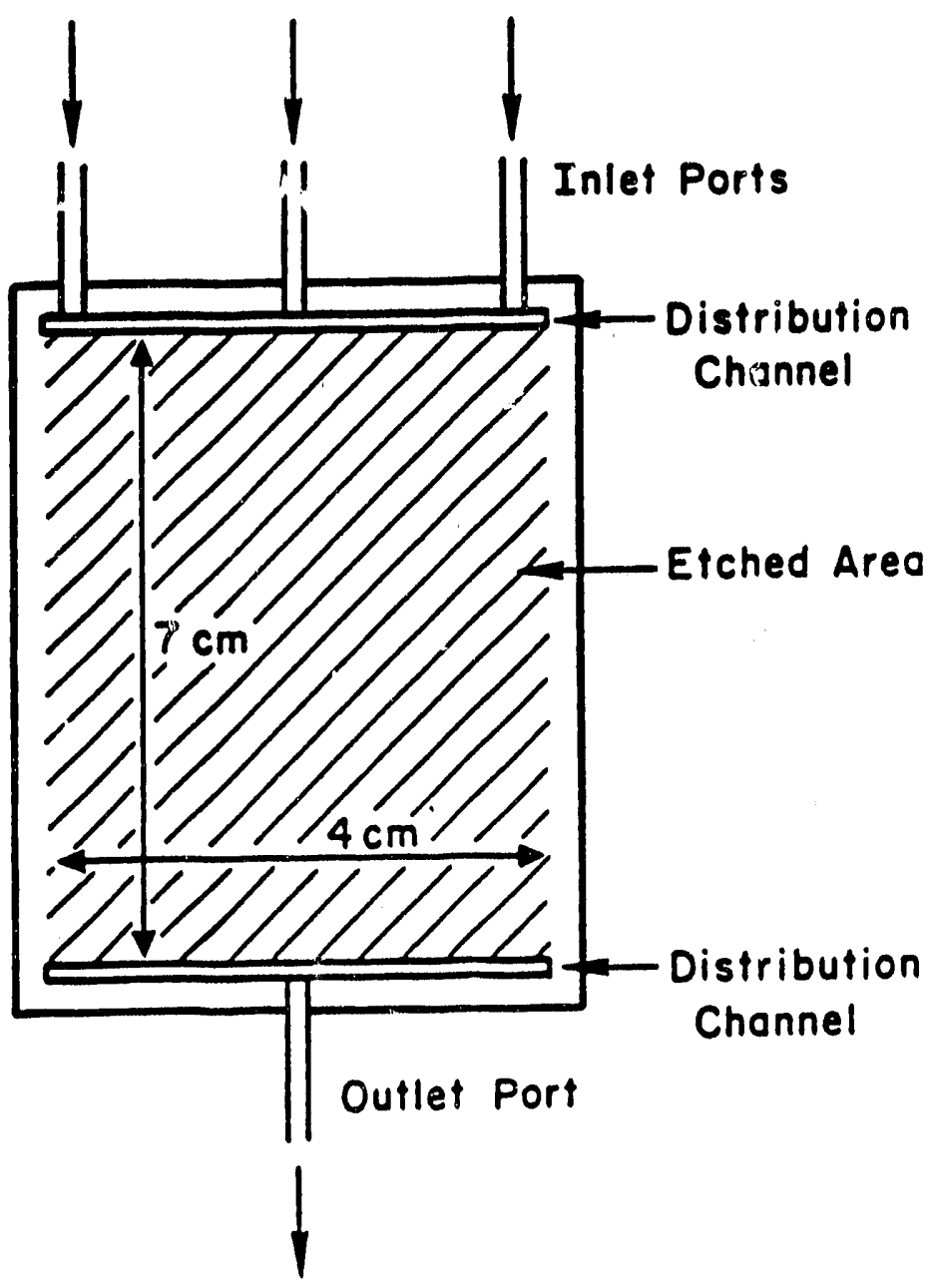




\section{Micromodel Specifications}

Figure 2 presents a photograph of one of the two micromodels used in this study. As listed in Figure 1, the dimensions of the area etched with the Kuparuk sandstone pattern are $7 \mathrm{~cm}$ in the axial flow direction by $4.4 \mathrm{~cm}$ across. The depth of the pores, determined by the width of the pores and the length of time allowed for the etching step (i.e., the wider the pores and the longer the etch time, the greater the depth of the pores), ranged from approximately $75 \mu \mathrm{m}$ to $125 \mu \mathrm{m}$ according to data provided by Adobe labs. The widths of the pores were measured under a microscope. The pore throats varied between 25 and $125 \mu \mathrm{m}$ while the pore bodies ranged from 150 to $300 \mu \mathrm{m}$ in width.

Manlowe [19] previously measured permeability and porosity values for the two micromodels. He developed a novel conductimetric technique to measure accurately very small pore volumes because the conventional approach of relying upon variations in the weights of an evacuated and a saturated porous sample is inadequate for determining the pore volumes of the micromodels. The results of his micromodel characterizations appear in Table 1 where the "event" micromodel refers to that containing the well-connected flow network and the "foam-generation" micromodel refers to that containing the less wellconnected flow network. Manlowe did not pursue an accurate determination of the pore volume or porosity in the foam-generation micromodel because he never utilized flow results for this model in a quantitative manner. He calculated single-phase liquid permeabilities for both models by measuring the steady-state pressure drops across them at a known volumetric flow rate of deionized water and by assuming that the average depth of the etched area in both models is $100 \mu \mathrm{m}$. Note that the permeability measurements in Table 1 verify the lower connectivity in the foam-generation micromodel. Both micromodels were utilized in this study of pore-level capillary instabilities. The wellconnected and the less well-connected micromodels will be referred to as the "event" and the "foam-generation" models, respectively, throughout the remainder of this work. 


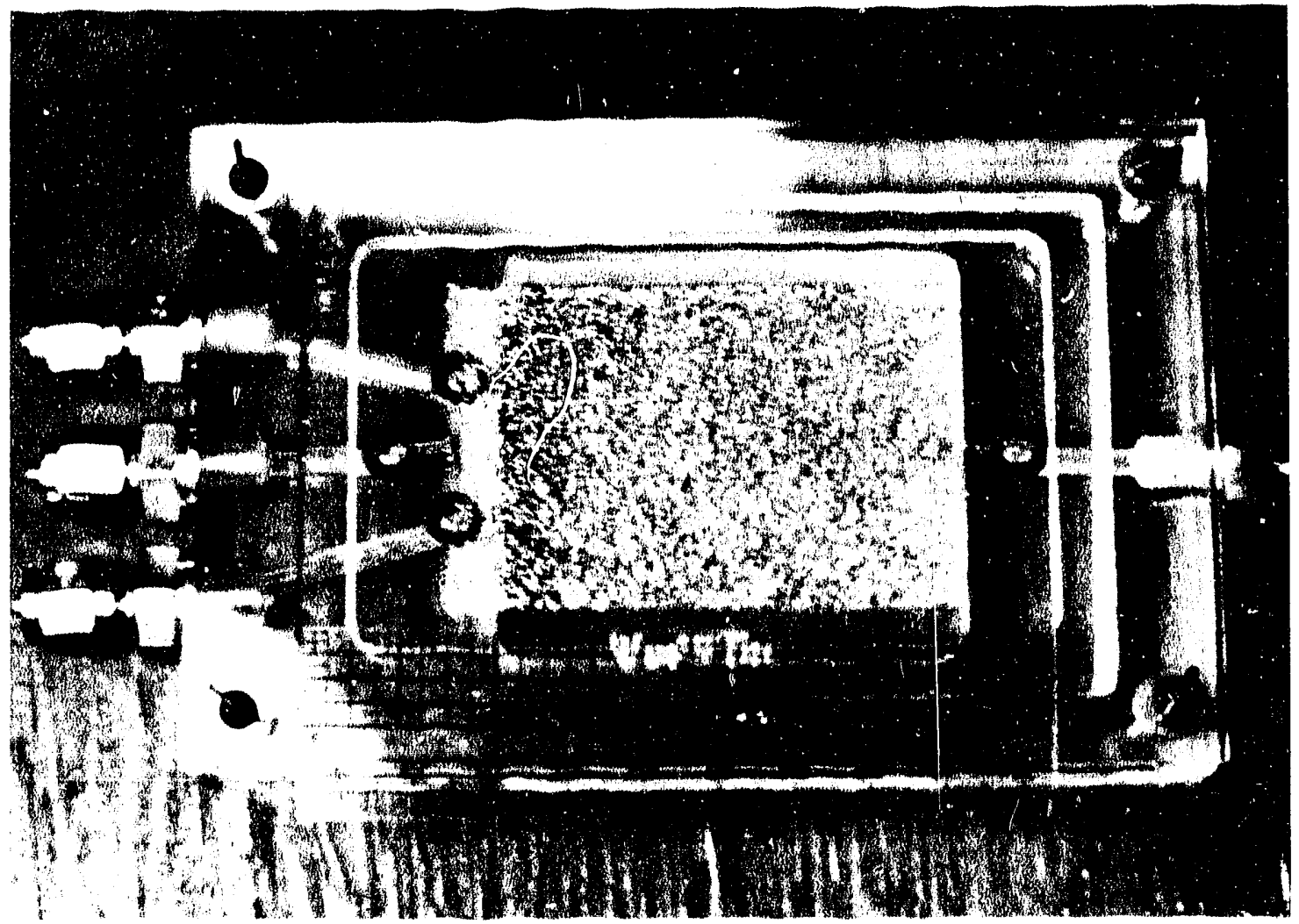

Figure 2: Photograph of micromodel. Scale provides a gauge of size. 
Table 1: Micromodel Specifications

\begin{tabular}{|l|c|c|}
\hline Characteristic & Event Micromodel: \\
\hline Areal Dimensions & $4 \mathrm{~cm} \mathrm{by} 7 \mathrm{~cm}$ & Foam-generation \\
\hline Pore Throats & $25-100 \mu \mathrm{cm}$ by $7 \mathrm{~cm}$ \\
\hline Pore Bodies & $25-100 \mu \mathrm{m}$ \\
\hline Permeability & $150-300 \mu \mathrm{m}$ & $150-300 \mu \mathrm{m}$ \\
\hline Pore Volume & $1.1 \mathrm{~mm}^{2}$ & $0.85 \mathrm{~mm}^{2}$ \\
\hline Furosity & $0.19 \mathrm{~cm}^{3}$ &.- \\
\hline 0.42 & -- \\
\hline
\end{tabular}

* based on an average pore depth of $100 \mathrm{\mu m}$. 


\section{Foam Flow Apparatus}

The experimental set-up used in this study facilitated both qualitative and a limited amount of quantitative aralysis of pore-level events in the porous-medium micromodels. Figure 3 diagrams the experimental set-up [19]. Two syringe pumps (Harvard, Model 975) delivered air and aqueous surfactant solution separately to the inlet ports in the foamgeneration micromodel. To alleviate problems encountered with air leakage around the syringe, aqueous surfactant solution was used in both pumps. But in one puinp the surfactant solution was used only to displace air from a small $\left(5 \mathrm{~cm}^{3}\right)$ air reservoir, as shown in Figure 3. Except for $40 \mathrm{~cm}$ total of $1 / 8$ inch, stainless steel tubing used as the inlet and outlet of the air reservoir, all of the flow lines used in this set-up consisted of $1 / 8$ inch, flexible teflon tubing. The surfactant solution was delivered to one inlet port in the foam-generation micromodel through $20 \mathrm{~cm}$ of teflon tubing while, in addition to the $40 \mathrm{~cm}$ of stainless steel tubing leading in and out of the air reservoir, the air was delivered to a separate inlet port in the same model with $10 \mathrm{~cm}$ of teflon tubing.

The foam-generation micromodel was attached vi $i_{i}$ a $T$-joint to both a pressure transducer (Validyne, Model DP12) and the downstream event model. The length of tubing between the foam-generation model and the transducer was $20 \mathrm{~cm}$ while the length between the two micromodels was $30 \mathrm{~cm}$. The pressure transducer was equipped with a diaphragm allowing for a maximum pressure drop across the model of $5 \mathrm{psi}$, and it was then placed approximately $10 \mathrm{~cm}$ from the inlet to the event micromodel. The 'ransducer recorded pressure relative to atmosphere. Since the outlet of the model was open to atmosphere and the length of tubing containing foam downstream of the transducer was very short $(10 \mathrm{~cm})$, the pressure transducer effectively read the pressure drop across the model. As pointed out by Owete [16], the existence of the etched inlet and outlet flow distribution channels contributes to the total pressure drop across the model during fluid flow experiments. No effort was made to subtract off this component of the pressure drop 


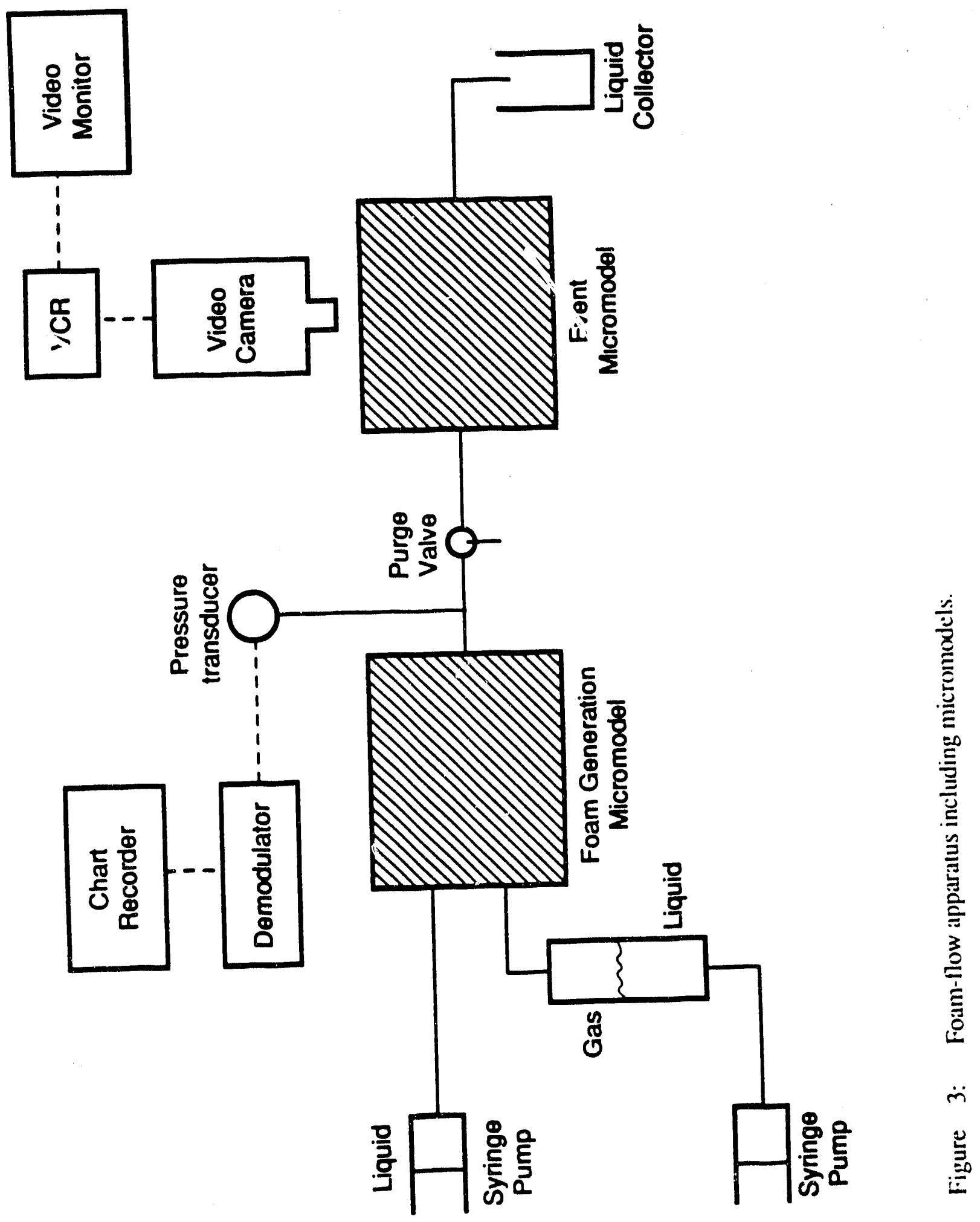


since the foam floods were primarily used in a qualitative sense. The pressure data were used primarily to ascertain that the foam flood had reached steady-state.

The transducer output was fed into a deinodulator (Validyne, Model CD12) while a chart recorder (Linear, Model 385) recorded the demodulated pressure sign`l. Modification of the connection between the demodulator and the chart recorder was required to utilize most effectively the full range of the chart recorder. The demodulator produced a maxi num output signal of $10 \mathrm{~V}$ while the recorder could only accept a maximum signal of $5 \mathrm{~V}$. Therefore, a voltage dividing circuit was placed between the demodulator and the chart recorder [19]. Figure 4 presenis the schematic of the circuit used for this purpose.

The most important aspect of the experimental set-up for studying pore-level events was its video-recording capability. The event micromodel was placed on a home-rnade microscope stand, which allowed for precise lateral and axial translation. The stand also has the capability to measure the exact $\mathrm{x}$ and $\mathrm{y}$ position of the micromodel with precision micrometers. The capability to return to a very specific site in the etched area of the micromodel was important in studying different pore-level events during the course of a foam flood. The range of the translational stage permits an entire view of the micromodel by a stationary camera (Panasonic, HD Digital 5000). Appendix A details the camera assembly including the lenses used in this study. The assembly provides for maximum versatility in the magnification of the micromodel. Both pore-level and overall views were possible with good resolution. The output of the camera was recorded by an industrial VCR (Panasonic, Model AG-6010) and displayed on a high-resolution color television monitor (Sony, Model PVM-1271Q-1371QM). This allows for real-time monitoring of the events in the model as well as slow-motion taping of events for future analysis. The VCR has taping and playback speeds ranging from approximately $1 / 30$ second per frame to 1 second per frame. The effluent from the event micromodel was collected in a graduated cylinder. Unfortunately, the small fluid volumes associated with the foam floods precluded an accurate analysis of the produced fluids. 
Chart Recorder Input



Demodulator Output

Figure 4: Voltage-dividing circuit (from Maniowe [î̄j]). 


\section{Experimental Procedure}

Many of the foam floods were performed by injecting air at $0.078 \mathrm{~cm}^{3} / \mathrm{min}$ and aqueous surfactant solution at $0.02 \mathrm{~cm}^{3} / \mathrm{min}$ into the foam-generation model through separate injection lines. This produced a foam of approximately $80 \%$ quality at a total superficial velocity of around $100 \mathrm{ft} / \mathrm{day}$. Attempts were made to reduce the advance rate to a few feet per day, which is a more realistic estimate of the velocity existing away from the wellbore in an oil reservoir. However, the Harvard syringe pumps would not steadily and accurately deliver fluids to the micromodels at rates much below those used here. The gas injection rates changed gradually due to compressibility as the pressure drop across the micromodels increased during the course of a foam flood. This occurred because the syringe in the Harvard syringe pumps advances at a constant rate to drive air out of the small air reservoir, which is being subjected to a gradually increasing downstream pressure. An attempt was made to utilize a modified mass-flow controller (Brooks, Model $5850 \mathrm{E})$ to supply air in order to alleviate the variations in gas flow rate caused by compressibility. Unfortunately, the mass-flow controller could not accurately deliver the very low flow rates required in this study. The downstream event model was disconnected at the inlet flow line by opening a purge valve during the initial stages of fluid injection into the foam-generation micromodel. Once the foam-generation micromodel reached steadystate, which was defined as that point in time where visual observations of the foam near the outlet of the model revealed no change in bubble velocity or texture over a period of approximately 10 minutes, the purge valve was closed allowing foam to enter the event model and the progress of the flood was monitored and recorded. The event micromodel was said to have reached steady-state when the pressure drop across the model stabilized and there was no indication of significant alterations in the foam texture near the outlet to the model. This typically occurred after several tens of pore volumes of foam had been injected into the event model. 
In addition to the procedure outlined above, several other injection schemes were used during the foam floods. For instance, pore-level events were recorded while injecting only air into a surfactant-saturated micromodel and, also, while injecting only surfactant solution into a nearly foam-saturated model following a foam flood. As a result, information on the injection mode, including the fluids used and the total superficial velocity, is included when referencing an illustration of a pore-level event in the remainder of this work.

In all cases, both the foam-generation and event micromodel were initially saturated with deionized water prior to initiating a foam flood. The models were then saturated with aqueous surfactant solution by displacing them with ten pore volumes of surfactant solution at the same total superficial velocity used in the ensuing foam flood. After a flood, both models were flushed with approximately 50 pore volumes of deionized water followed by 50 pore volumes of isopropanol (to break the remaining foam) and then another 50 pore volumes of deionized water.

\section{Chemicals}

Only one surfactant was utilized in this work. In all cases, the aqueous surfactant solution consisted of either 0.005 or 0.5 wt $\%$ active $\mathrm{C}_{14-16} \alpha$-olefin sulfonate surfactant (BioTerg, Stepan) in deionized water. The gas phase in all cases was air.

\section{Capillary Phenomena in Micromodels}

\subsection{Overview}

Micromodels accurately reproduce many of the important physical characterislics of naturally-occurring porous media. For example, they reflect reasonable dimensions for the flow channels (see Table, 1), converging and diverging pores, a distribution of porethroat and body sizes, nooks and crannies for wetting liquid to flow in channels containing 
primarily nonwetting fluid, and a network structure capable of transmitting information on capillary pressures on both a pore-level and a more macroscopic scale. As a result, the capillary phenomena that dictate the occupancy of the flow paths by the wetting and nonwetting fluids, as well as determine the magnitude of the capillary pressure, govern the micromodel flow behavior. Likewise, the pore-level capillary events observed during micromodel foam flonds provide a realistic picture in many cases of the pore-level events which exist during foam flow in naturally-occurring porous media.

Although micromodels are valuable in representing capillary phenomena, they do have limitations in accurately representing all of the characteristics of multiphase flow in naturally-occurring porous media. Perhaps the most obvious limitation is that the glass matrix in the models fails to reproduce the complicated chemistry and mixed wettability associated with most reservoir rocks. Consequently, the importance of contact-angle values or hysteresis $[21,22]$ are not addressed in this work. The very small pore volumes of the micromodels precludes an accurate scaling of foam flow behavior in the models to predict flow behavior on a reservoir scale due to the existence of capillary end effects [23], trapping of ons phase against the impermeable side boundaries in the models [17,24], and the absence of three-dimensional connectivity between pores [17]. These considerations should not affect the physics underlying individual pore-level events occurring away from the boundaries of the models, however, because these events are not directly influenced by boundary effects or the lack of three-dimensional connectivity with neighboring pores. All of the examples of pore-level events presented in this thesis were recorded away from the boundaries of the micromodels. Two additional considerations that could significantly affect the interpretation of capillary events observed in the micromodels, on the other hand, are the absence of distinct flow networks for both the wetting and nonwetting phases at steady-state flow conditions $[18,25]$ and the difficulty in extrapolating the two-dimensional observations to three-dimensional behavior $[17,18]$. The lack of distinct, steady-state flow networks is a consequence of percolation considerations in two-dimensional lattices. 
The elementary concept of a percolation threshold is needed at this time in order to explain this behavior.

\subsection{Application of Percolation Concepts to Micromodels}

Percolation theory was developed by Broadbent and Hammersley [26] to predict transport properties in disordered media. A disordered medium is characterized by a random spatial distribution of connected flow paths of different conductivities. Larson et al. [27] noted that if "the fluid flow paths are determined by the medium, but the medium itself is in some sense random, the process is called a percolation process." Percolation has found a wide range of applicability in describing multiphase flow behavior in porous media because the pore throats and bodies, which are of varying sizes and therefore have different conductivities, are often randomly distributed throughout the media [24, 27-31]. Most percolation calculations are performed on either regular lattices or on Bethe trees. A regular lattice is a collection of sites (nodes) and bonds (branches) with each end of a bond being attached to a site and with each site being attached to the same number of bonds, i.e, the coordination number of the lattice is constant. A regular Bethe tree is an infinite graph, which, like a regular lattice, has a coordination number common to all sites. The fundamental difference between a regular lattice and a regular Bethe tree is that the former has infinite connectedness since between two sites there is an infinite number of distinct connecting pathways of bonds, whereas the latter has a connectedness of unity because there is one and only one pathway of bonds connecting any given sites. An example of a regular lattice and a regular Bethe tree, both with a coordination number of 4 , appears in Figure 5 .

The advantage of Bethe trees over lattices is that analytical solutions of percolation problems can be obtained for the former, whereas the latter usually require time-consuming Monte Carlo computer calculations. Percolation is usually applied in one of two fundamentally different ways to describe 


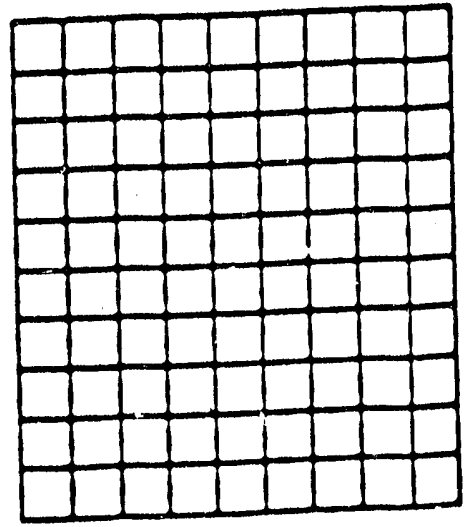

(a)

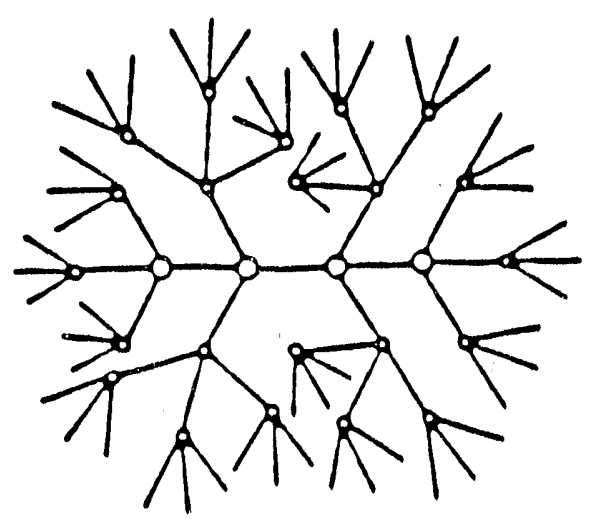

(b)

Figure 5: Schematic comparing (a) regular lattices to (b) Bethe trees. Both figures represent a coordination number of 4 . 
transport in a random lattice or tree. Under the first approach, referred to as bond percolation, all of the transport properties are assigned to the bonds in the iattice or tree while the sites merely act as junctions for the bonds. With the second approach, called site percolation, all of the transport properties are assigned to the sites and tie bonds only act to connect the sites together. Variations on the above two schemes exist, but they are much less common [32].

The Kuparuk-sandstone flow network eiched into the micromodels used in this study can be viewed as a two-dimensional lattice, albeit an irregular one, because the coordination number at each site, i.e., pore body, is not constant. Assuming that the pore throats control the fluid occupancy during the drainage mode (which exists in almost all of the foam floods performed in this study) and that the throat sizes are randomly distributed throughout the network, then bond percolation considerations can be applied to describe multiphase fluid transport during the foam floods. Another important parameter in percolation calculations, in addition to deciding on either the bond or the site mode, is the coordination number of the lattice.

The coordination number in porous media represents the number of throats (bonds) connected to a body (site) in the medium. A detailed review of the etched, Kuparuk-sandstone pattern indicates an average coordination number of approximately 4 , which is the same as that for a regular square lattice.

During a bond percolation calculation, a fraction $\mathrm{p}$ of the bonds, which are randomly distributed throughout the lattice, are occupied. The occupied bonds are considered conducting while the remaining unoccupied bonds are considered nonconducting. The percolation threshold or probability $\mathrm{p}_{\mathrm{c}}$ refers to the fraction of the total bonds that are occupied in the lattice when a conducting path first appears from one side of the lattice to the other. Percolation theory actually addresses the conductivity of infinite systems, but computer simulations on finite lattices indicate the same percolation behavior as long as the lattice is sufficiently large $[24,25,29,32,33]$. Simulations of bond- 
percolation behavior performed by Chatzis and Dullien [25] for two-dimensional lattices having a range of coordination numvers indicated that a lattice width of 20-35 bonds and a length of greater than 40 bonds gives good agreement with tabulated bond percolation thresholds for infinite two-dimensional lattices. The network pattern etched into the micromodels used in this study does exceed these minimum dimensions and, therefore, the micromodels should exhibit percolation behavior representative of an infinite medium. The one major difference between the actual micromodel behavior and percolation considerations is the existence of trapping of one of the phases against the impermeable side boundaries in the micromodels. Regular percolation theory does not account for this behavior. Invasion percolation $[24,29]$ can account for the existence of trapping.

The bond percolation threshold on the square lattice is $1 / 2$. In terms of multiphaseflow behavior, half of tine bonds must be occupied by a particular phase before that phase can flow from one side of the lattice to the other. Analogous limiting saturations, i.e., bond occupancies, appear for a water-wet porous medium as $S_{w, i r r}$ and $\mathrm{S}_{\mathrm{nw}, \mathrm{irr}}$. Since the probability of having exactly half of the throats in the micromodel occupied by the wetting and nonwetting phases at steady-state conditions during a foam flood is very small, the likelihood of having distinct flow networks for both phases is also small. In fact, careful review of the micromodels during steady-state flow conditions did not reveal the exis ence of a continuous flow network for the wetting phase. This was true because the nonwetting (foam) saturation was well in excess of that associated with only half of the pore throats, leaving the wetting phase without a sample-spanning flow network. The lack of a continuous wetting network across the micromodels at steady-state does have an impact on some of the capillary events observed in the models because significant amounts of wetting liquid must then be transported through flow channels containing primarily the nonwetting phase. No analytical solution has been derived from the Navier-Stokes equations for the case of simultaneous, creeping, two-phase flow 
through a constricted noncircular capillary, which is the situation existing in the micromodels at steady-state due to the absence of a separate flow network for the wetting phase. Legait [34] did numerically model this behavior, however; for the case of nonwetting drops traveling through constricted, noncircular capillaries under the influence of either a constant wetting-liquid flow rate or pressure drop and within the domain of the lubrication approximation to the Navier-Stokes equations. The lubrication approximation is based on the premise that the slope of the constriction is small, i.e., less than :pproximately $20^{\circ}$ [35]. Although he was primarily concerned with mubilization criteria for nonwetting drops, Legait did note that flow of the wetting liquid along the corners of noncircular capillaries can lead to capillary instabilities at the wetting-nonwetting interface. Ransohoff et al. [36] also studied this phenomenon with regard to the formation of foam bubbles at pore constrictions which fall within the domain of the lubrication approximation, but they did not account for an externally applied pressure gradient in the wetting liquid. The ability of wetting liquid to destabilize an interface must be taken into account when analyzing the capillary instabilities observed in the sieady-state foam floods.

\subsection{Extrapolating 2-D Micromodel Observations to 3-D Behavior}

In addition to the percolation considerations discussed above, the validity of extrapolating two-dimensional observations of capillary events in the micromodels to describe three-dimensional behavior is a concern. The reason for this concern is that the third dimension, which cannot be viewed in the micromodels, can control capillary phenomena because the effective diameter for a noncircular flow channel depends on both its width and depth [13-15]. The effective diameter for a noncircular capillary corresponds to that diameter relating the capillary pressure $P_{c}$ to the surface tension $\sigma$ and contact angle $\theta$.

Lenormand et al. [13] calculated the effective diameter $d_{e}$ for a capillary of rectangular cross section for the case of a contact angle of $0^{\circ}$ as 


$$
d_{e}=\frac{2 F(e)}{\left(\frac{1}{x}+\frac{1}{y}\right)}
$$

where $F(e)$ is a function which is approximately equal to 1.0 and $x$ and $y$ are the width and depth, respectively, of the channel. Unfortunately, only $x$ can be seen in the plan view of the micromodels.

An accurate determination of the effective diameters for the complicated pore network present in the micromodels used in this study was not undertaken due to the dificulty in measuring the depth of the etched channels. Fortunately, Wardlaw et al. [37] did accurately characterize the flow channels in several different etched-glass micromodels by measuring all three dimensions of the etches with a Lietz optical microscope. A crosssectional view of a channel etched in a glass plate, taken from their work, appears in Figure 6. The flow channels in the micromodels used in this thesis should be similar to the one pictured in Figure 6 because they are of comparable size, and they were also created by etching glass plates with concentrated hydrofluoric acid. $\mathrm{Yu}$ and Wardlaw [15] demonstrated that Eqn. ( 1) can also be applied to the elliptic cross sections present in etched-glass micromodels where $\mathrm{x}$ and $\mathrm{y}$ are the principal axes of the ellipse. Using Eqn. ( 1), Wardlaw et al. [37] calculated the effectve diameters for the flow channels in several etched-glass micromodels. Results of their study for the effective diameter versus the width of an etch for several micromodels appear in Figure 7. The symbols correspond to data points for four different micromodels, denoted by $\mathrm{A} 1$ through $\mathrm{A} 4$, and the lines represent the least squares fit of the data for $d_{e}$ versus $x$ based on the best fit obtained with either a linear or a logarithmic relationship between the two. Micromodel A2 contains pores with both square and triangular shapes when viewed from above. The data in this figure indicate that larger widths correspond to larger effective diameters, although the effective diameter does increase more gradually than the width. This is especif ' true at large values of the width, as indicated by a flattening of curves $A 1$ and $A 3$ in Figure 7 at 
large widths. Data on the depth of an etch in a glass micromodel is needed to understand this behavior.

Figure 8, also taken from the same study and for the same four micromodels, indicates that the width of an etch, $x$, is usually much greater than the corresponding depth, y. This observation is consistent with the pore illustrated in Figure 6. Furthermore, the depth increases very slowly with the width. Apparently, the reason for this behavior is that a reaction product formed during the hydrofluoric-acid etching process inhibits further reaction of the glass. Eqn. ( 1 ) indicates that the smaller channel dimension controls the effective diameter. The fact that depth increases slowly with width in the glass micromodels explains the gradual increase in effective diameter with increasing width that is illustrated in Figure 7. An important point to note in this analysis is that the effective diametcrs shown in Figure 7 pertain to flow channels etched in a single glass plate. The micromodels used in this study, on the other hand, consist of two fused glass plates, each plate having been etched with a mirror image of the flow network in the other. Consequently, the depth of an etch is much closer to the width of an etch in these micromodels, and the correlation between increasing width and effective diameter is expected to be much better than that shown in Figure 7. As a result, there appears to be justification for extrapolating the two-dimensional observations in this study to describe three-dimensional capillary phenomena. In other words, wide pores correspond to large effective diameters and iow capillary entry pressures while narrow pores correspond to small effective diameters and high capillary entry pressures.

A brief

review on the importance of the pore structure as it relates to capillary phenomena is discussed in the next section prior to presenting the results of the foam flood experiments. 


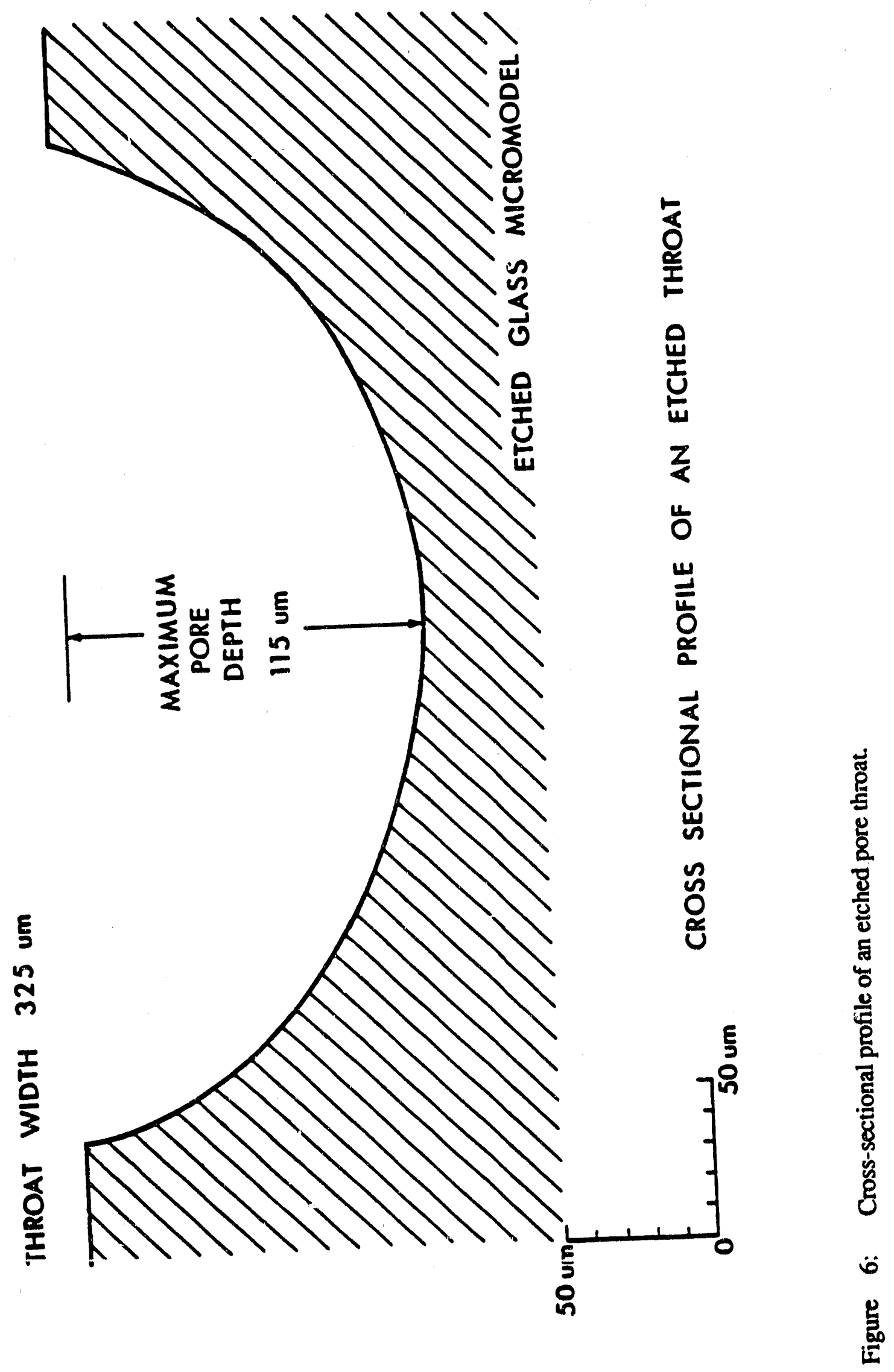




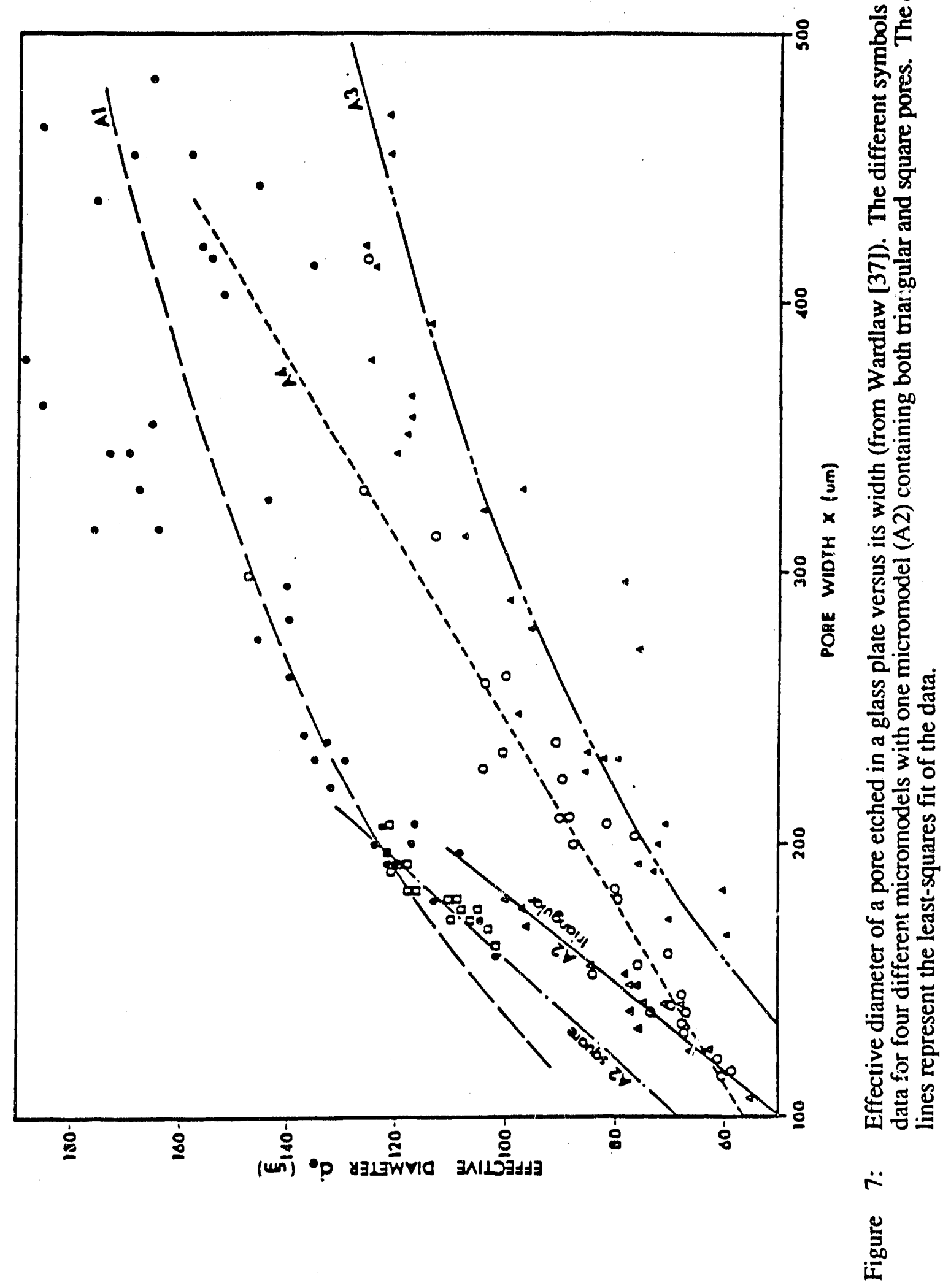




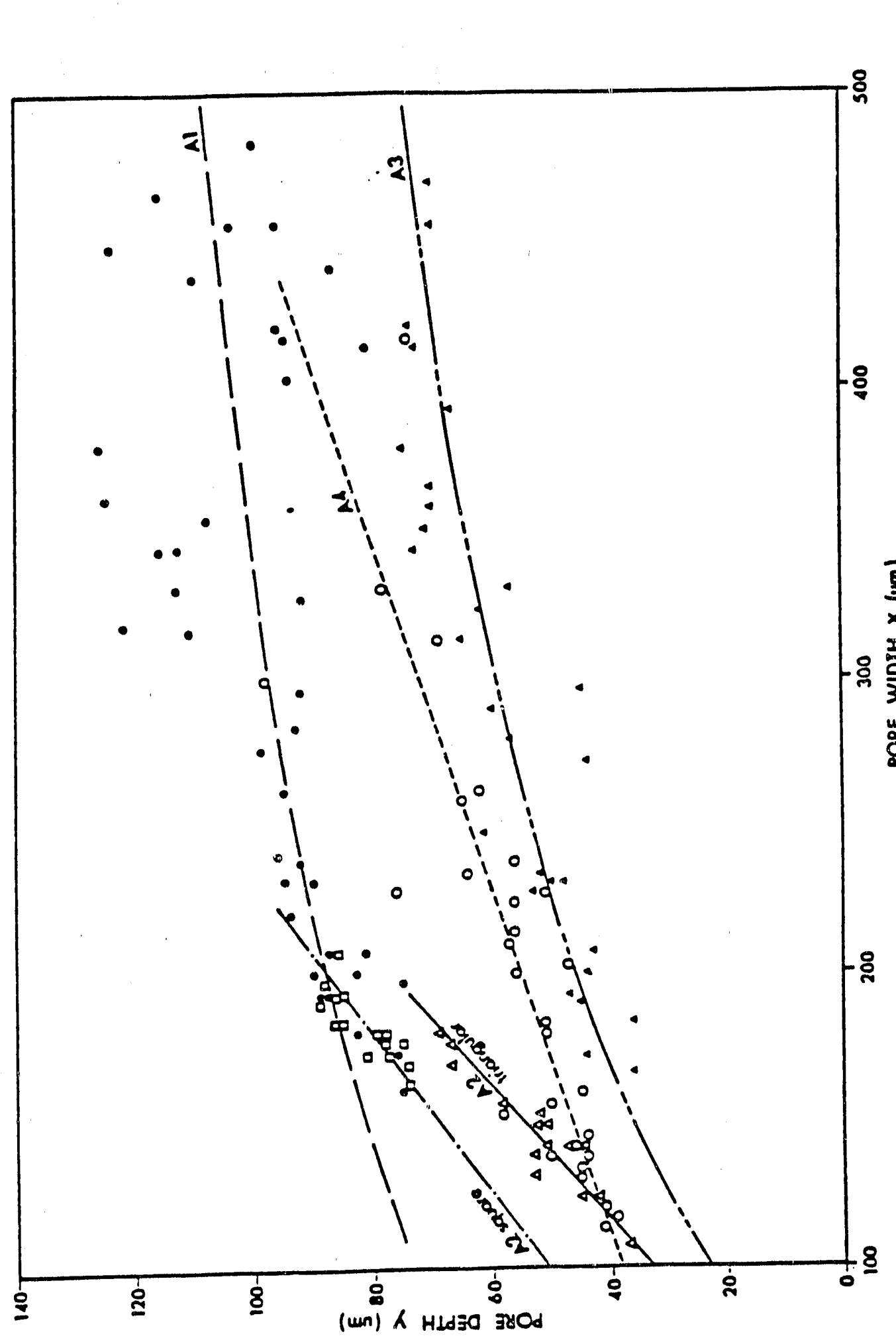

马्乛.

월

긍

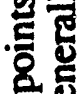

요

용.

룽음

है।

它

요

눌

ज活

ํㅗㄹ

- $3^{\frac{1}{4}}$

훈

$x$

$\div 3$

官

옹 웡

공

急

는

퐁

$>$ 용

氠

-

os

.5 독

$8 \%$

ปิ

ป ปั

눙 웡

\%

प응

专起

$\ddot{\infty}$

馜 


\section{Importance of Pore Structure on Capillary Instabilities}

Pore geometry plays an important role in determining the stability of fluid interfaces in porous media. The following discussion on capillary instabilities addresses specifically the case where gas is the nonwetting and liquid is the wetting phase because this is almost universally the system of interest to foam flow in porous media. Furthermore, the glass micromodels used in this study exhibit these same wetting characteristics. Figure 9 illustrates the effect of a small axisymmetric perturbation of wavelength $\lambda$ on the local liquid satura ${ }^{i}$ (i.e., film thickness) in a circular capillary containing a gas thread surrounded $b_{j}$ a thin wetting film of original thickness $h_{0}$ and perturbed thickness $h . A$ thin film such as the one illustrated in this figure is deposited when a long bubble or droplet of nonwetting fluid flows slowly through a circular capillary filled with wetting fluid [38]. The thickness of the film and the magnitude of the disturbance have been greatly exaggerat/d in this figure to facilitate the discussion of capillary instabilities. The thickness of the th in film is several orders of magnitude smaller than the capillary radius at flow rates existing in an oil reservoir away from wells. The increase in the liquid saturation in the vicinity of point 1 results in an increase in the mean curvature along the perturbed interface compared to the curvature in the unperturbed interfaces existing to the left and right of this reprion.

An increase in the mean curvature $\mathrm{C}_{m}$ corresponds to an ir crease in the capillary pressure $P_{c}$. Assuming an inviscid gas thread, the gradient from a higher capillary pressure near point 1 to a lower value in the unperturbed regions causes wetting liquid to flow into the perturbed region thereby increasing its curvature even further. The local increase in liquid saturation results in the formation of a wetting collar. Everett and Haynes [39] and Mohanty [40] showed that a liquid collar can only support a critical volume before it rearranges into a lens in order to meet the thermodynamic constraint of minimizing the surface free energy. Âs discussedi in more dêtâu iñ ã lâtễ section on foam generation, liquid lens formation is the precuitsor to lamella formation. 


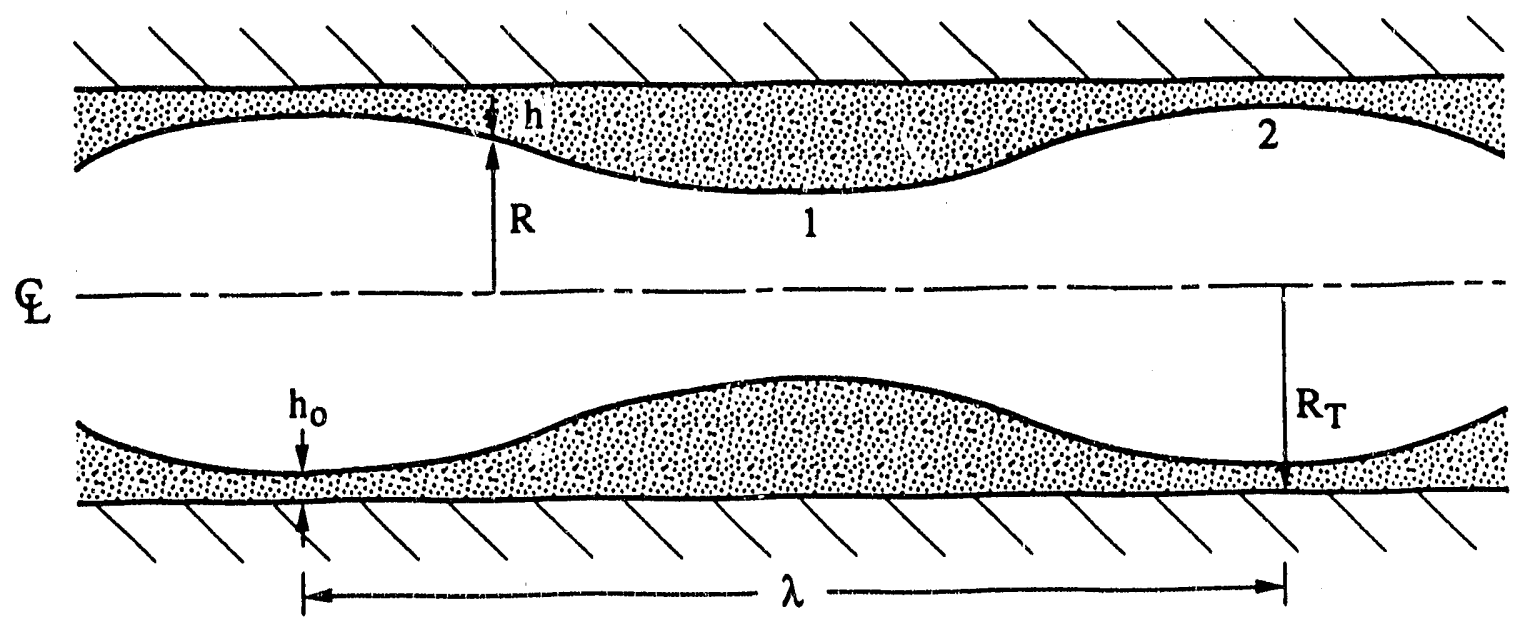

Figure 9: Schematic of capillary-driven flow induced by an infinitesimal disturbance of wavelength $\lambda$ in a cylindrical capillary containing an inviscid nonwetring thread. Higher curvature at 1 compared to 2 leads to a capillary-pressure gradient that drives liquid toward 1 resulting in the formation of a liquid collar. 
Gauglitz and Radke [41] recently demonstrated that a lens will form in a circular capillary by the mechanism discussed above if the original film thickness is greater than about $12 \%$ of the capillary radius. In the absence of sufficient liquid to form a lens, Hammond [42] showed previously that the liquid film accumulates at periodic intervals along the capillary into isolated collars. Aul and Olbricht [43] studied the stability of thin wetting films surrounding less viscous nonwetting fluids in small $\left(50 \times 10^{-6} \mathrm{~m}\right)$ circular and square capillaries during pressure-driven, core-annular flow experiments. The coreannular flow regime more accurately represents multiphase flow characteristics in porous media than the initially static conditions assumed to exist in the first two studies mentioned above. The experiments indicated that axisymmetric lobes formed in the wetting liquid at periodic intervals for initial film thicknesses down to approximately $4 \%$ of the capillary radius. The lobes then moved with a small phase-velocity along the capillary tube. The lobes continued to grow until they formed a lens across the capillary and pinched-off the inner core. Pinch-off occurred at periodic intervals along the capillary at a wavelength of approximately $2^{3 / 2} \pi R_{T}$ where $R_{T}$ is the radius of the tube. This wavelength corresponds to the fastest-growing wavelength from a linear stability analysis [43]. Although the authors did not perform a stability analysis in the case of the square capillaries, the experimental observations from the square capillaries also indicated the growth of collars and eventual pinch-off of the gas thread at periodic intervals of approximately $23 / 2 \pi R_{T}$ where $R_{T}$ is the half-width of the square cross section. The reason that Aul and Olbricht's experiments indicated breakup of the rionwetting core fluid for initial film thicknesses of less than $12 \%$ of the capillary radius was that wetting liquid was continually supplied to their system along the annular film as opposed to the experiments of Gauglitz ani Radke [41] for a fixed initial volume of annular wetting fluid.

An important point to note in all of the studies and calculations for the stability of axisymmetric gas threads is that the thread is stabie to periurbations below a critical wavelength. This critical wavelength, from linear stability analysis, is $2 \pi R_{T}$ where $R_{T}$ is 
the radius of the capillary [41-44]. Consequently, an additional requirement for breakup of a gas thread is that the capillary which confines it has a length greater than the critical wavelength and can therefore support long-wavelength disturbances. This also sets a minimum size for a foam bubble created by this mechanism that is approximately three times the pore diameter. As pointed out by Aul and Olbricht [43] and Ratulowski and Chang [44], stable gas threads will exist in channels with lengths less than $2 \pi R_{T}$.

Although often modeled with circular capillaries, the flow channels in porous media are usually quite angular. The piłotographs in Figure 10, taken from thin-sections of Berea sandstone following impregnation by molten Wood's metal at increasing capillary pressure [45], reveal the irregular cross section of the flow paths. The nonwetting Wood's metal (light shading) recedes farther into the corners of the flow channels as the capillary pressure increases. This is shown by the increased angularity of the pore space invaded by Wood's metal between Figure 10a, which corresponds to a Wood's-metal saturation of $73 \%$, and Figure 10b, which corresponds to an increase in the Wood's-metal saturation to $90 \%$. The existence of corners in porous media influences both the stability of fluid interfaces and the rate that any instabilities grow at these interfaces.

In contrast to gas threads in circular capillaries, nonaxisymmetric gas threads in noncircular capillaries are stable to small perturbations in the local liquid saturation over a limited range of values. An increase in the liquid saturation as shown by the dotted line in Figure 11a, even neglecting the stabilizing influence of the transverse (out of the page) radius of curvature, reduces the mean curvature of the interface. The initial and perturbed interfaces are denoted in this figure by 1 and 2 , respectively. The reduced curvature corresponds to a lower capillary pressure which translates to a higher liquid pre ssure for the case of an inviscid gas thread. Consequently, liquid flows out of this region thereby returning the liquid saturation to its previous value. This behavior holds uniil the liquiud sāturation reaches the critical value depicted in Figure 11 b. Any further increase in the liquid saturation results in the unstable behavior discussed above, assuming 




(a)

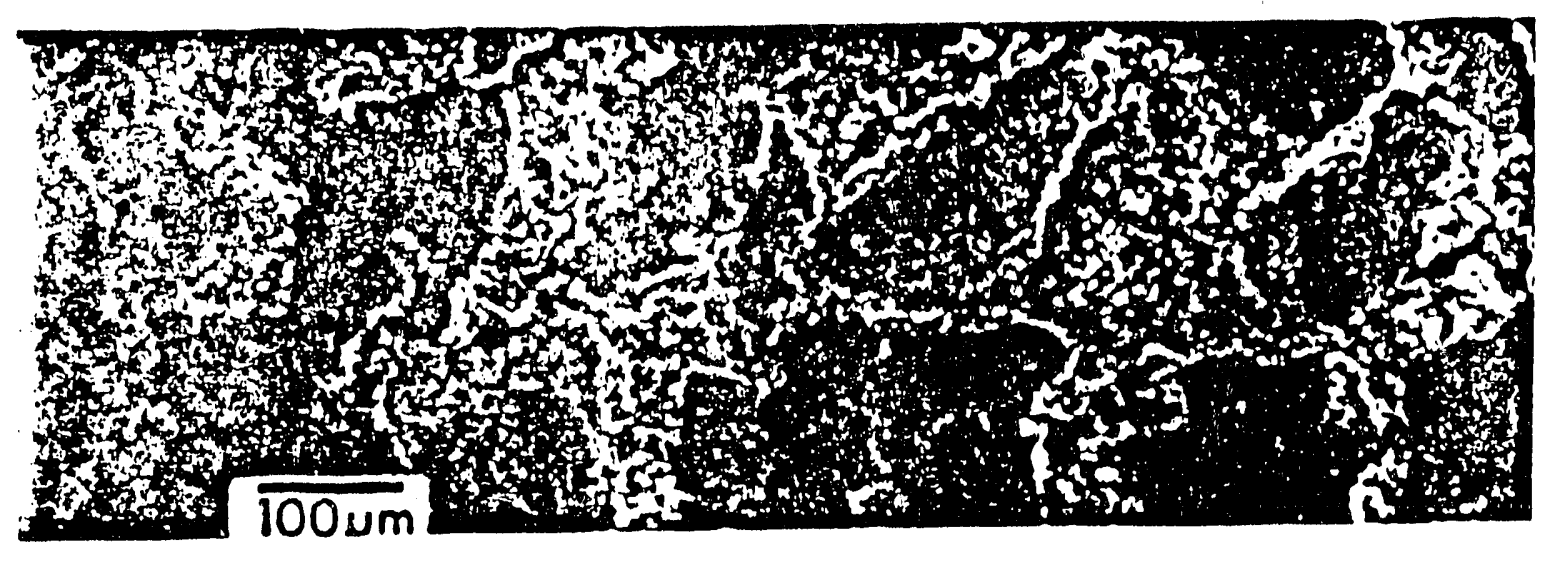

(b)

Figure 10: Micropholographs of Wood's metal (light shading) occupying (a) $73 \%$ and (b) $90 \%$ of the pore volume in Berea sandstone (from Wardlaw et al. [45]). The rock (üark shauding) was removed by dissolution in acid. 



Figure 11: Stability of nonwetting threads in noncircular capillaries showing (a) stable and (b) unstable configuration resulting from a local saturation disturbance (dotted line). The initial and perturbed principal radii of curvature are $R_{1}$ and $R_{2}$, respectively. 
that the pore can support a long-wavelength disturbance, and pinch-off of the gas thread ensues.

The photographs in Figure 10 indicate that the nonwetting phase should be relatively stable to breakup at high saturations of the nonwetting phase because it assumes stable curvatures in the corners of the noncircular flow channels. It then follows that lamellae generation is insignificant at the high nonwetting-phase saturations typically measured in steady flow experiments with strong foams $[2,3,7,46]$. Observations of foam textures at high foam saturations, on the other hand, indicate that lamellae generation is very active in these systems $[5,7]$. The weakness in extrapolating the pictures of nonwetting-fluid distributions in Figure 10 to predict the stability of immiscible fluid interfaces relates to the dynamics of the flow processes in porous media. The variable cross sections of the flow paths in porous media and the flow of wetting fluid along the corners of predominantly gas or foam-occupied pores introduce pore-level capillary instabilities during dynamic conditions that create new fluid interfaces. Examples of these pore-level capillary instabilities resulting in lamellae generation and destruction are presented in the remainder of this report.

\section{Lamellae Generation Mechanisms}

\subsection{Snap-off}

Investigators have identified several mechanisms for lamellae generation in porous media. An illustration of in important mechanism, termed the snap-off process, appears in Figure 12. Roof [47] first identified and explained this phenomenon with regard to the snap-off of oil droplets in toroids. As demonstrated by the irregular cross section of the flow charinels in Figure 10, noncircular, constricted capillaries provide a reasonably realistic framework for studying flow behavior in porous media on the pore level. The capillary pressure increases in response to an increase in the curvature of the interface as the 


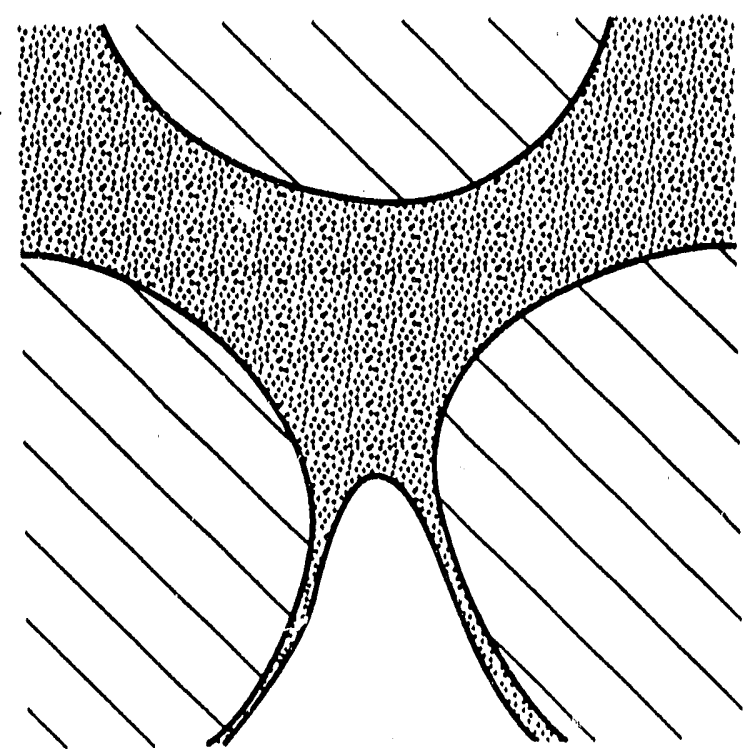

(a)

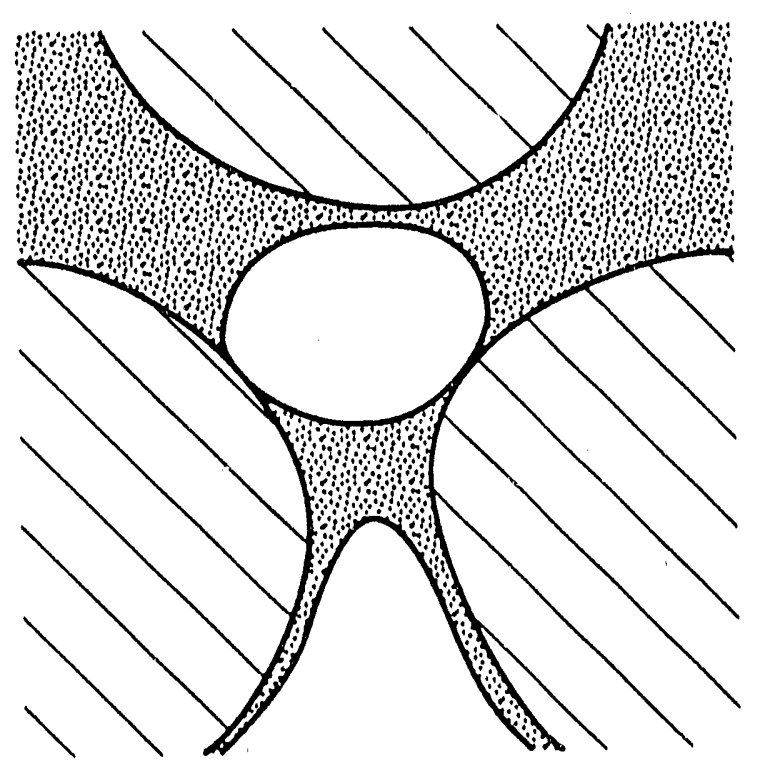

(c)

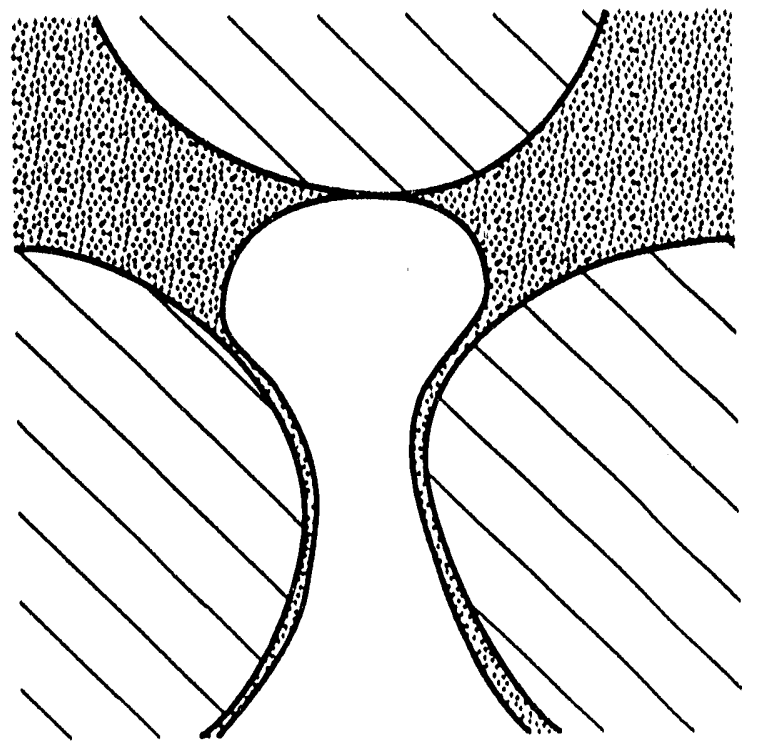

(b)

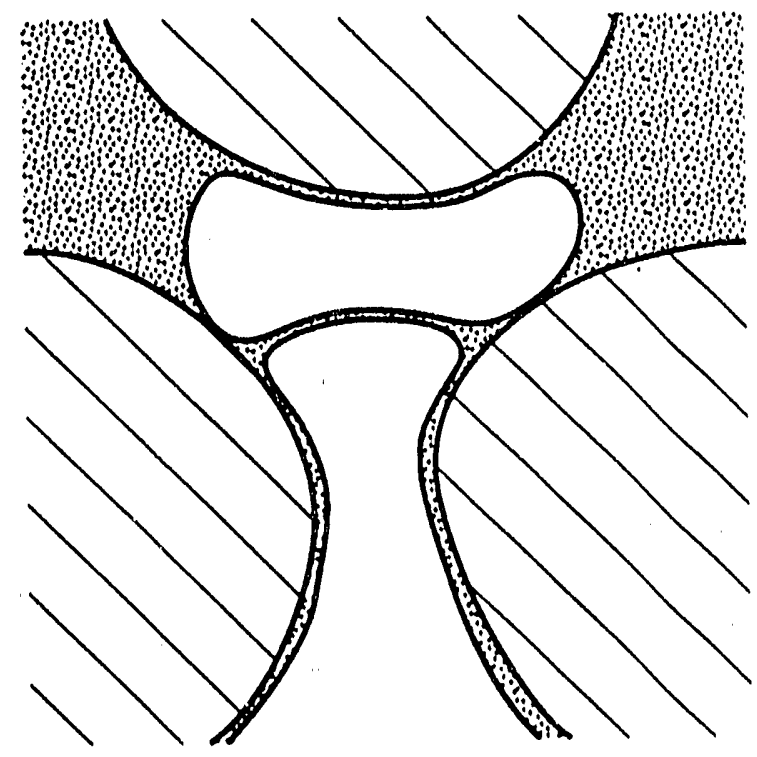

(d)

Figure 12: Schematic of snap-off in a constricted, noncircular capillary: (a) gas enters pore constriction, (b) gas front expands into downstream pure body setting up a driving force for liquid flow, (c) gas bubble snaps-off leaving a liquid lens, (d) a lamella forms as gas reinvades the pore constriction. 
nonwetting gas bubble enters the noncircular pore throat in Figure 12a. The curvature and thereby the capillary pressure at the bubble front then begin to fall as the bubble expands into the pore body, as shown in Figure 12b. Since the gas phase is assumed to be inviscid, this gradient in capillary pressure corresponds to a gradient in liquid pressure that drives liquid toward the pore throat. The curvature in the throat decreases as liquid begins to accumulate there. Snap-off occurs if, as ine result of liquid drain back, the curvature in the throat falls to the value corresponding to the unstable axisymmetric gas thread pictured in Figure $11 \mathrm{~b}$.

The snap-off process results in the formation of a gas bubble separated from the original gas finger by a liquid lens. A new lamella is generated, as shown in Figure $12 \mathrm{~d}$, as gas reinvades the pore throat and drives liquid out of the lens that was created as the bubble snapped-off. Lens formation always precedes lamella formation because a wetting collar can support a volume of liquiu approximately equal to $1.8 \pi \mathrm{R}_{t}^{3}$ prior to breakup (i.e., snap-off), based on a thermodynamic stability analysis [39-41], where $R_{t}$ is the constriction radius. A lamella forms as the local capillary pressure increases in response to gas reinvading the pore throat and snapping-off more gas bubbles. The size of a foam bubble created by this mechanism is of the order of the size of a pore body.

Snap-off occurs during multiphase flow in porous media whether or not surfactant is present. The presence of surfactant has a profound impact on the flow behavior, however, because surfactants stabilize interfaces that otherwise would coalesce immediately. Any attempt to estimate foam rheoiogy should incorporate dynamic pore-level events describing lamellac generation due to snap-off $[3,5,7,10-12]$ because the stable lamellae that result from this process in the presence of surfactant lead to shear-thinning and yield-stress, i.e., trapping, behavior in the gas phase.

Roof [47] developed a static geometric criterion to predict snap-off in constricted circular capillaries as a nonwetting fluid enters a capillary filled completely with wetting liquid. Ransohoff et al. [36] extended the analysis to consider the dynamics of the snap-off 
process in noncircular capillaries. They solved the hydrodynamics for liquid flow in the corners of noncircular capillaries in order to determine the time for enough wetting liquid to accumulate in the throat to allow snap-off. The snap-off time in noncircular geometries proves to be an important element in calculating the rate of lamellae generation in porous media $[3,11]$.

A critical value in determining the snap-off time is the thickness of the initial liquid deposit that results as a gas bubble advances through a capillary filled with a wetting liquid $[36,38,44,48]$. Ransuhoff et al. [36] and Gauglitz et al. [48] pointed out the fundamental difference between circular and noncircular capillaries in regard to this thickness. Figure

13 compares the bubble-deposited liquid profiles for the two cases. Based on Bretherton's analysis [38], the dimensionless film thickness in a circular capillary, expressed as the ratio of the film thickness $h$ to the tube radius $R_{T}$, varies with the capillary number $\mathrm{Ca}$ to the two-thirds power (for $\mathrm{Ca} \ll 1$ ):

$$
\frac{h}{R_{T}}=O\left(\mathrm{Ca}^{2 / 3}\right)
$$

The dependence of the thin-film thickness on the capillary number remains in the case of noncircular flow geometries $[44,48]$. In addition to thin films, however, thicker liquid accumulations appear in the corner regions of noncircular channels, as shown in Figure 13b. The amount of liquid in the corner regions at equilibrium is available in the literature for several regular geometries $[13,34,36,49]$. In smoothly consiricted capillaries where the longitudinal curvature is much less than the axial curvature, the equilibrium radius of curvature $R_{m}(z)$ is directly proportional to the radius of the iargest inscribed circle $R(z)$ at each axial position $z$ in the capillary, as shown below:

$$
R_{m}(z)=\frac{R(z)}{\widetilde{C}_{m}}
$$




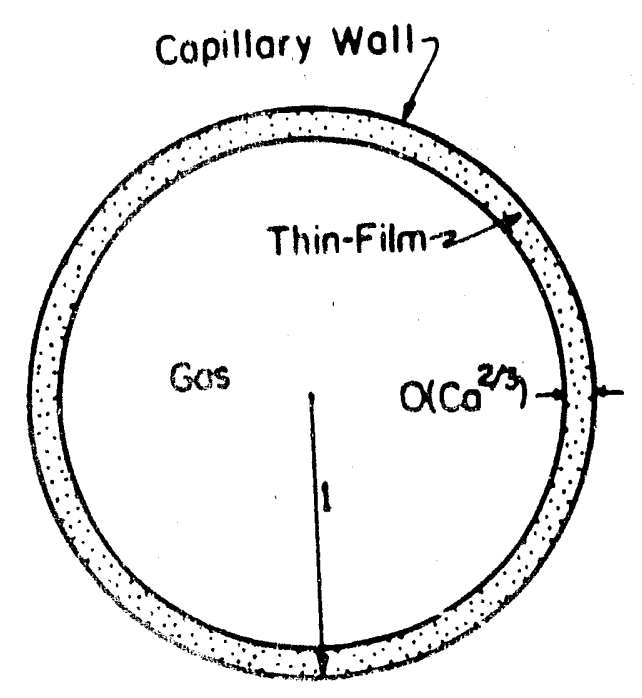

a

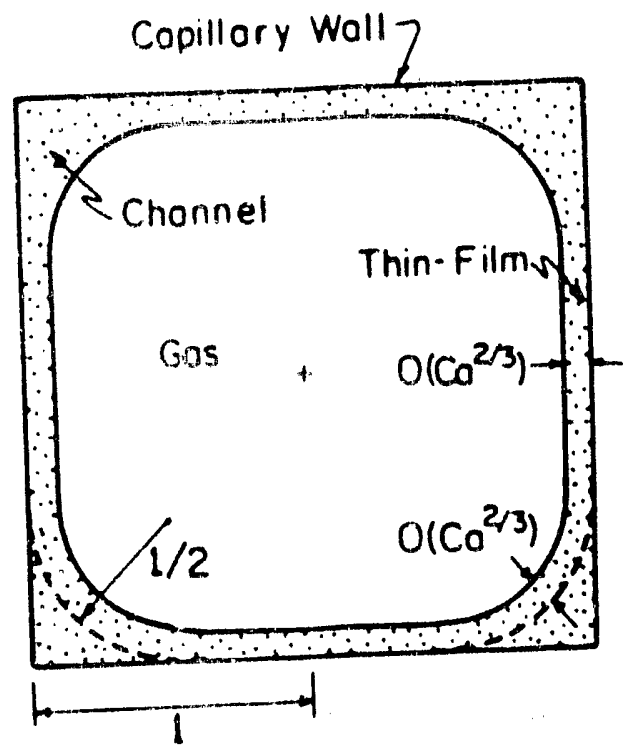

b

Figure 13: Schematic of bubble-deposited liquid profiles in (a) cylindrical and (b) square-cross-sectiona! capiliaries. The valuies of ! and !/, correspond to the normalized curvarures for cylindrical and square cross sections, respectively. 
where $1 / \widetilde{\mathrm{C}}_{\mathrm{m}}$ is a dimensionless proportionality constant, which is approximately equai to $1 / 2$ for several geometries of interest $[13,34,36,49]$. Consequently, the value of $1 / 2$ in Figure 13b corresponds to the dimensionless radius of curvature $R_{m}(z) / R(z)$ in this square geometry. At the low capillary numbers expected to exist during most oil recovery projects $\left(\mathrm{Ca}<10^{-4}\right)$, the thin-film contribution toward the snap-off time in the noncircular flow paths of the porous medium is not expected to be significant [48], whereas it controls the snap-off time in circular capillaries $[44,50]$. Furthermore, the equilibrium curvature in noncircular pores, which is set by capillary forces, will not be altered significantly by viscous forces due to flow because the capillary forces dominate in porous media at low Ca.

In addition to its role in determining the bubble-deposited liquid profiles important to the dynamic of the snap-off process, the value of $1 / \widetilde{C}_{m}$ also serves as the geometric criterion for snap-off in noncircular capillaries. As discussed earlier, snap-off occurs in noncircular geometries once the gas-liquid interface reaches the configuration shown in Figure $11 \mathrm{~b}$. This configuration is reached in the throat first where $R(z)$ is smallest. Neglecting the longitudinal curvature under the assumption of a smoothly constricted capillary, the critical value for the curvature in the throat, $2 \mathrm{C}_{\mathrm{mt}}{ }^{*}$, then follows;

$$
2 \mathrm{C}_{\mathrm{mt}}{ }^{*}=1 / \mathrm{R}_{\mathrm{t}}
$$

where $R_{t}$ is the radius of the largest inscribed circle in the throat. Assuming local equlibrium, the curvature in the pore body, $2 \mathrm{C}_{\mathrm{mb}}$, follows from Eqn.

$$
2 C_{m b}=\widetilde{C}_{m} / R_{b}
$$

where $\mathbf{R}_{b}$ is the radius of the largest inscribed circle in the pore body. Snap-off occurs if the curvature in the body falls to or below the critical curvature in the throat. With Eqns. 
( 4) and ( 5), the inequality $C_{m b} \leq C_{m t}{ }^{*}$ can be rewritten to give the following expression:

$$
R_{b} \geq \widetilde{C}_{m} R_{t} .
$$

This criterion states that if the radius of the pore body is greater than the right side of Eqn. ( 6), then snap-off occurs; otherwise a stable configuration results and the gas thread remains intact. Practically, this means that the size of a pore body must be about twice that of the connected pore throat to allow snap-off.

Observations of foam flow in transparent micromodels indicate that snap-off is an important mechanism for generating lamellae when the pore bodies in the medium are much larger than their attached throats $[12,16-18]$. In addition to the criterion for snap-off expressed in Eqn. ( 6), visualization of foam flow in micromodeis, unlike that in isolated capillaries, reveals the importance of the pore network structure as it responds to fluctuations in the local capillary pressure. The isolated, constricted-capillary model is helpful for uncovering the hydrodynamics describing lamellae generation and coalescence. The capillary-tube model, unfortunately, does not reflect the high degree of pore connectivity which characterizes porous media. The large number of connecting paths among pores and the flow of wetting fluid along their corners transmits capillary pressures throughout the medium.

Falls et al. [11] also noted the importance of the local capillary-pressure environment on snap-off in beadpack experiments. They observed that snap-off times increased and that snap-off eventually ceased altogether at a low-to-high permeability transition in their pack as the local capillary pressure increased. Shirley [17] noted similar snap-off behavior as the gas saturation increased during a micromodel study of high pressure $\mathrm{CO}_{2}$ foam.

The microphotographs in Figure 14, recorded in the current micromodel flow study, illustrate a typical snap-off event. This sequence was taken while injecting air at a 




(a)

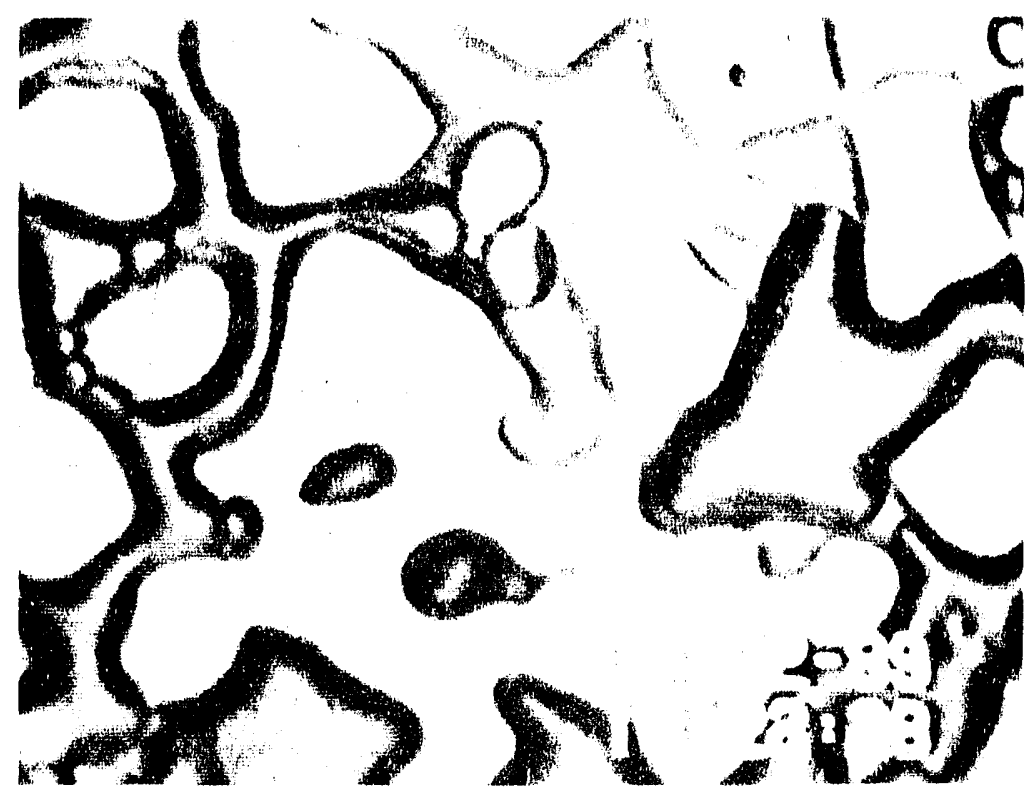

(b)

Figure 14: Microphotographs of snap-off recorded during gas (air) injection into an etched glass micromodel, which was originally saturated with surfactant solution, at a superficial gas velocity of $13 \mathrm{~m} / \mathrm{day}$ : (a) gas enters pore throat

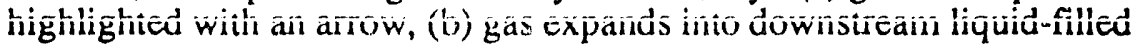
pore body, 


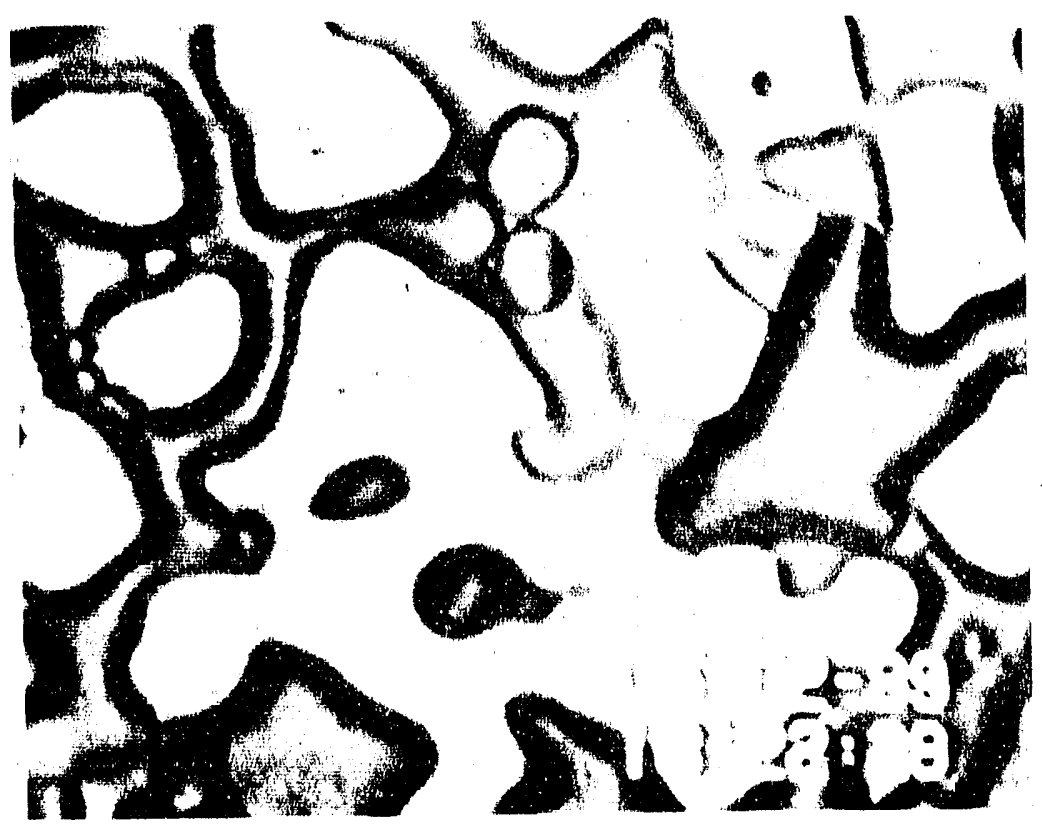

(c)

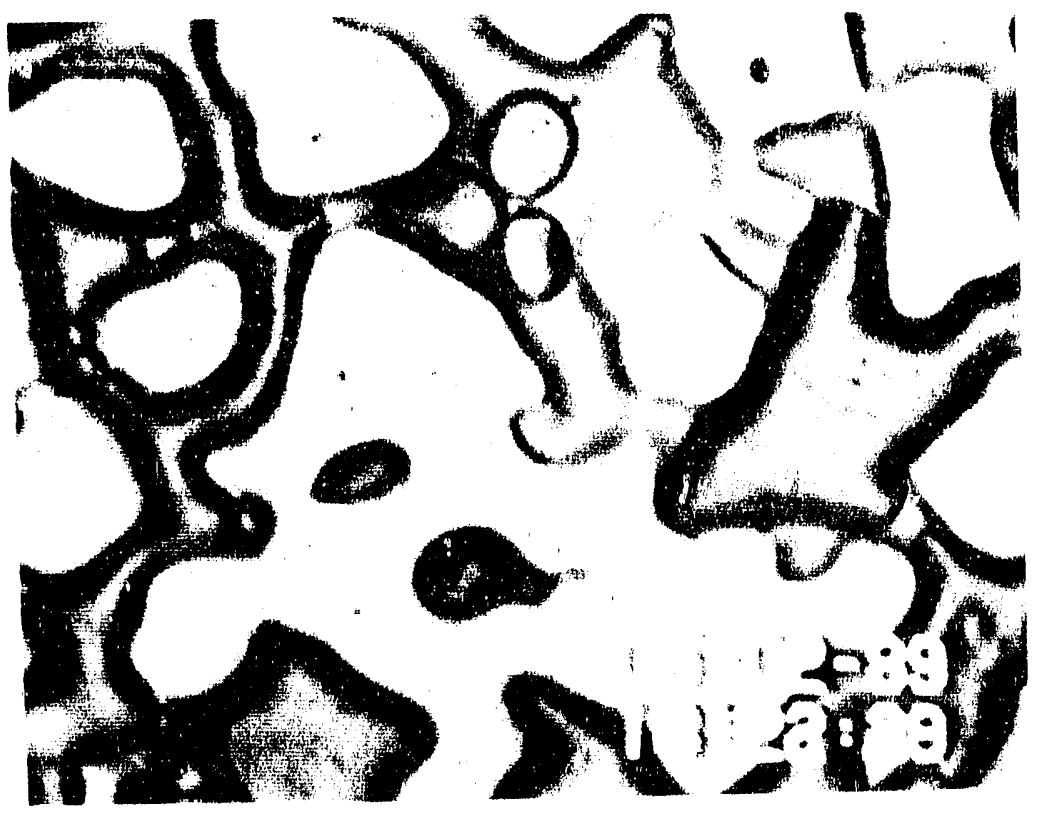

(d)

Figure 14: (c) formation of iiquid coliar iit thie pôrc throat, (d) snap off occurs less than (cont.) 1/30 of a second after the collar appears in (c). 
superficial velocity of $13 \mathrm{~m} /$ day into the event micromodel, which was originally saturated with a $0.5 \mathrm{wt} \%$ active surfactant solution. Gas enters a liquid-filled pore throat and then expands into the neighboring pore body in Figures $14 \mathrm{a}$ and b. As discussed earlier, differences in the curvature of the gas-liquid interface between the body and throat cause wetting liquid to flow along the corners of the noncircular pore and into the throat. A collar first appears in Figure $14 \mathrm{c}$ indicating that the axisymmetric thread configuration has been reached in the throat. The microphotograph in Figure 14d, taken 1/30 of a second later, shows that snap-off occurs soon after this unstable configuration is reached. The total elapsed time between Figures $14 \mathrm{a}$ and $14 \mathrm{~d}$ is 2.4 seconds. Note that the size of the bubble formed by snap-off in this sequence is on the order of the size of a typical pore body in the micromodel as are two of the three other bubbles that appear in this same large pore body. These two bubbles were also formed by snap-off, whereas the one small bubble that appears in the same pore body was formed by lamella division at the junction of several pore throats that exists to the left of the snap-off site. Lamella division is discussed in detail in Section 9.3.

Several important timercales apply to the snap-off process including the time to convect the bubble front to that point in the pore body where the geometric constraint for snap-off is first met (Eqn. ( 6)), the time for liquid to convect into the throat, and the time for the axisymmetric thread to snap-off in the throat. Calculations for breakup times of axisymmetric threads $[44,50]$ are in agreement with the experimental observation noted above that snap-off occurs almost immediately upon the formation of an unstable gas thread. Consequently, either liquid drainback into the pore throat or gas convection into the pore body controls the rate of snap-off in porous media $[36,50]$. The latter of these two times pertains to the snap-off sequence illustrated in Figure 14 based on an estimate of the drainback time that is on the order of $0.1 \mathrm{~s}$. 
A few seconds after the event just discussed, gas reinvades the same throat and forms another bubble, as shown in Figure 15. The total elapsed time for this second snap-off event is 2.5 seconds. A short time later, another bubble snaps-off in the same pore body. The fourth gas finger to invade this throat, however, does not snap-off, as shown in Figure 16. This behavior can be understood by comparing the curvature of the bubble front in Figure $16 \mathrm{c}$ to the curvatures of the bubble fronts in Figures $14 \mathrm{c}$ and

15b. The increasing gas saturation in this pore body with successive snap-off events results in an increase in the local capillary pressure which translates to a higher curvature at the bubble front. Eventually the curvature in the pore body increases to such an extent that there is no longer a curvature difference driving liquid into the throat and snap-off ceases. The criterion for snap-off expressed in Eqn. ( 6) assumes that gas expands freely into a water-filled capillary. As the gas saturation and capillary pressure increase in the porous medium, this relationship must be modified to account for the influence of the capillary environment $[10,11]$.

Note that lamellae begin to appear in Figure $16 \mathrm{~d}$ as the local gas saturation and capillary pressure increase in response to repeated gas invasion and snap-off at this location. The micromodel observations of liquid-lens generation followed by lamella formation with an increase in the local capillary pressure are in agreement with the thermodynamic analysis for the stability of a liquid collar which predicts a relatively thick liquid index upon breakup of a nonwetting thread [39-41].

Without injecting liquid containing surfactant into the flow system, snap-off will only be important in generating foam bubbles during the transient stage of the flow process as gas enters liquid-filled snap-off sites. Observations of steady-state foam flow in this micromodel study indicate that liquid regenerates snap-off sites by sporadically displacing gas from some of the pore throats in the network. Some time later, gas reinvades the liquid-filled throats and a bubble snaps-off. This behavior occurs both at pore throats iocated along the backbone fraction of the foam flow network and at pore 


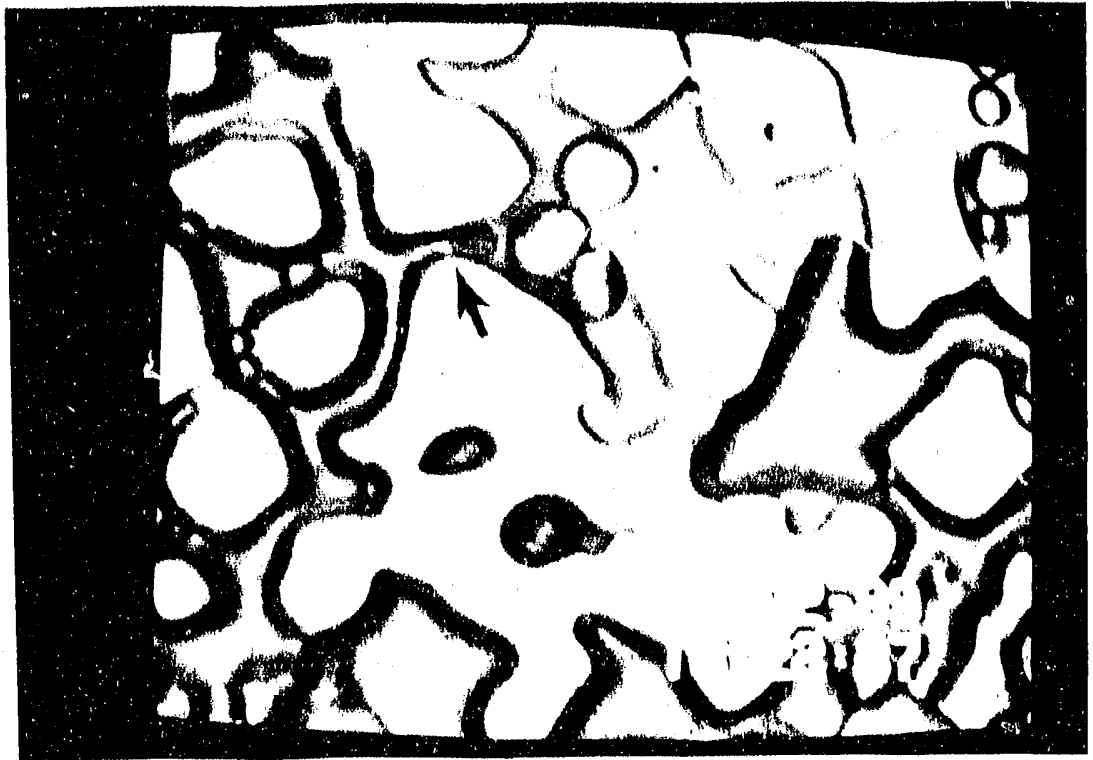

(a)



(b)

Figure 15: Microphotographs of snap-off repeating itself in the same location and at the same flow conditions discussed in Figure 14: (a) gas enters pore throat, (b) gas expands into downstream pore body, 


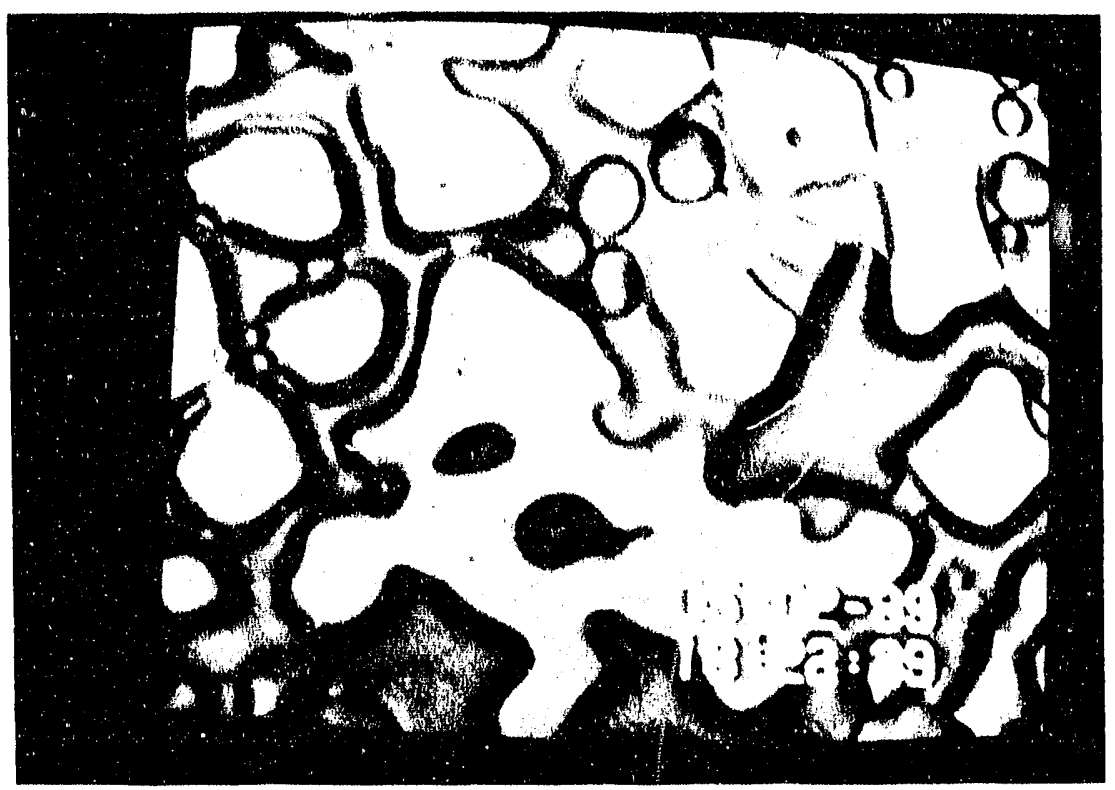

(c)

Figure 15: (c) gas bubble snaps-off. (cont.) 


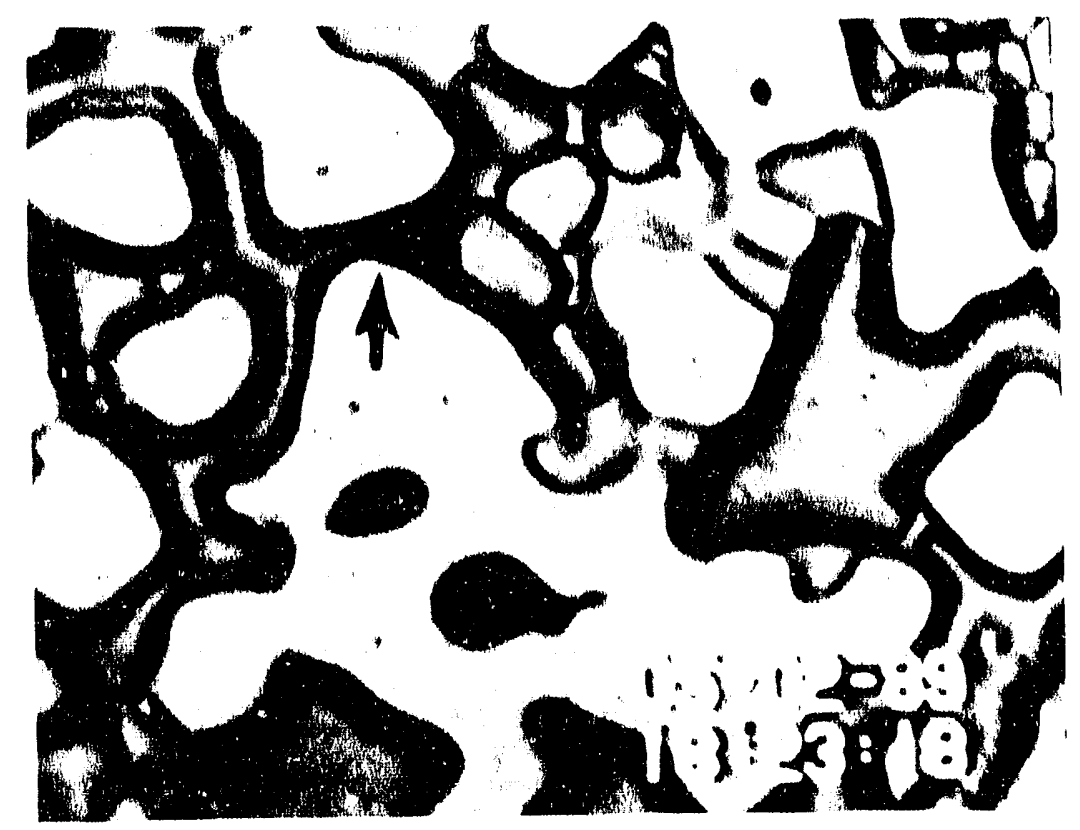

(a)

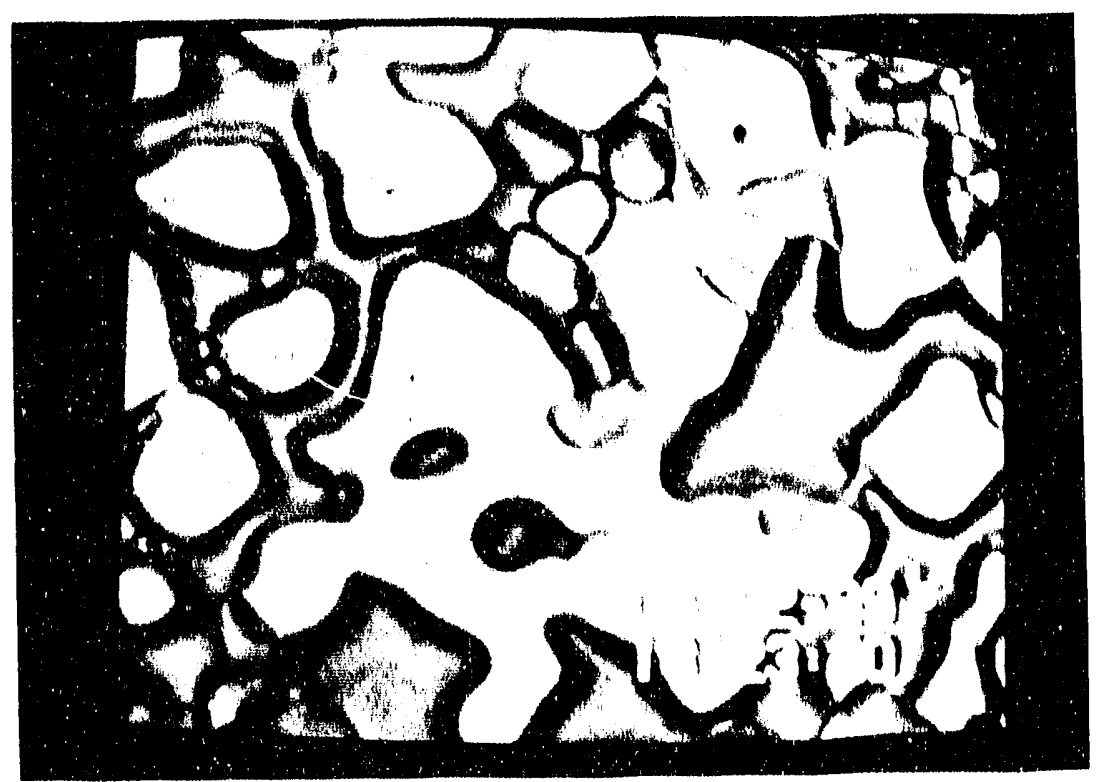

(b)

Figure 16: Microphotographs showing the deactivation of the snap-off site discussed in Figures 14 and 15 due to an increase in the local gas saturation and capillary pressure: (a) gas enters pore throat, (b) curvature at gas front is modified (increased) by increased gas saturation in downstream pore body. 


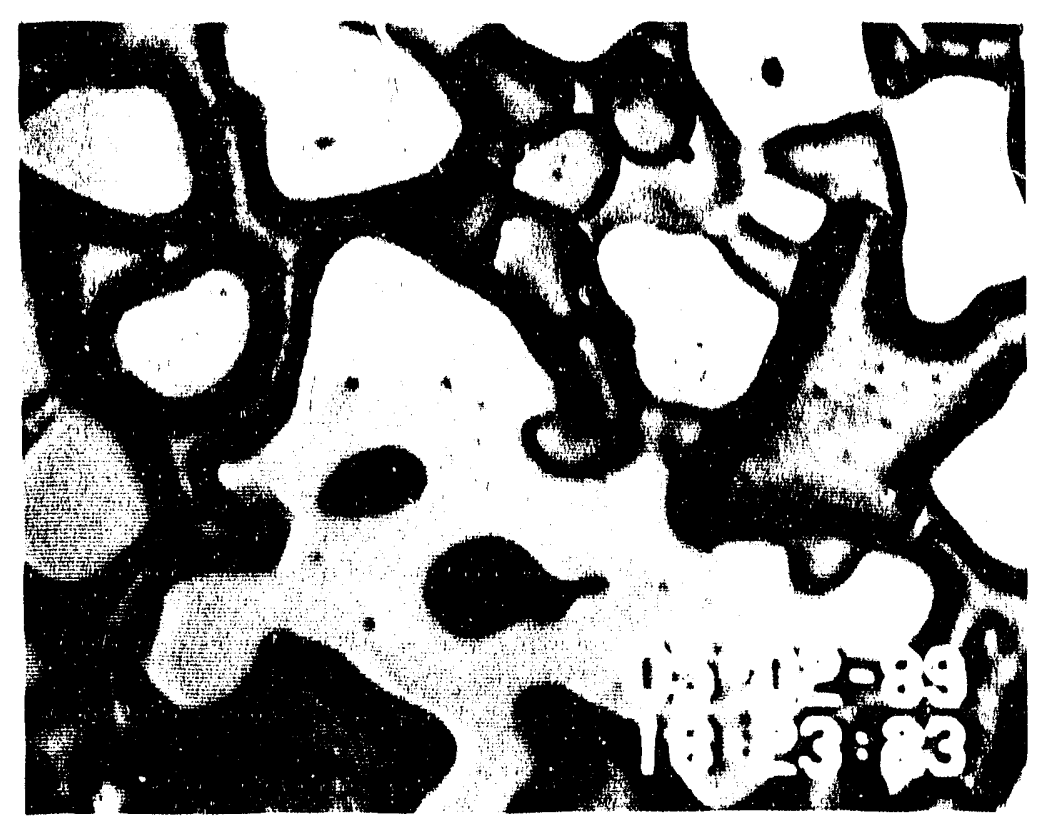

(c)

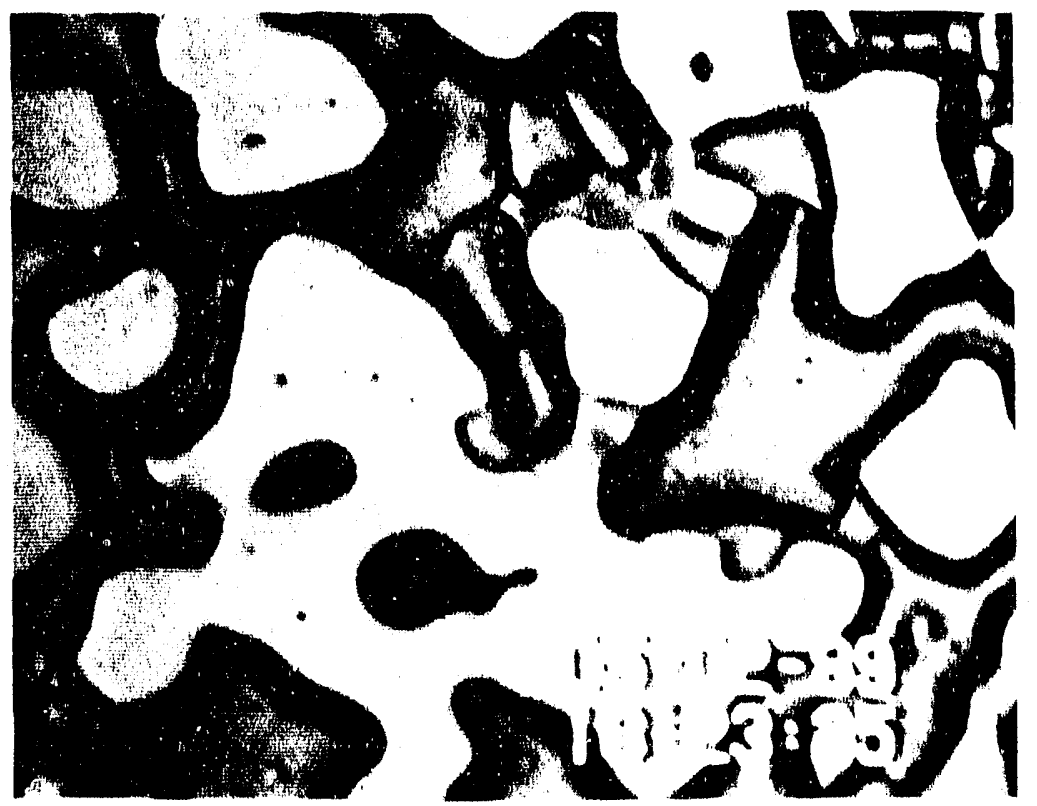

(d)

Figure 16: (c) continued increase in curvature at bubble front, (d) gas completely fills (cont.) pore body without snapping-off. 
throats within the short clusters of pores leading off of the backbone fraction which contain alternating trapped and flowing trains.

The microphotographs in Figure 17 illustrate the intermittent snap-off process. They were recorded in the event model at steady-state while injecting air and $0.5 \mathrm{wt} \%$ active surfactant solution at a total superficial velocity of $62 \mathrm{~m} /$ day and a gas fractional flow of 0.8. Liquid initially occupies the pore throat indicated with an arrow in Figure 17a, as shown by the intermediate shading there compared to the light background for the glass matrix and the dark shading for the gas, but gas enters from the upper left and displaces the liquid a short time later. This is shown by the dark shading that now appears in the pore throat of interest in Figure 17\%. The general direction of flow in the micromodel during this sequence is from left to right in the figure. Although difficult to discern in Figure

$17 \mathrm{~b}$, the original videotape clearly shows that gas bridges the two pore bodies connected to the throat that is highlighted with an arrow. Figure $17 \mathrm{c}$, taken four seconds later, shows that liquid imbibes into the left portion of the pore throat and snaps-off a gas bubble. This behavior is clearly not consistent with the snap-off event described in the preceding paragraphs. Neither the drainback time nor the time to convect gas into the pore body can account for the long lag between gas entering the pore throat and subsequently snappingoff. Consequently, an additional mechanism or mechanisms must explain this snap-off

behavior. Possible explanations are the role of wetting-fluid flow in the corners of primarily gas-occupied pores or local variations in the capillary pressure in destabilizing fluid interfaces. The comer flow of wetting fluids relates to weeping-flow behavior.

\subsection{Weeping-Flow Breakup}

The flow of wetting fluid in the corner regions of noncircular pores around trapped or slowly moving nonwetting bubbles or droplets is referred to as weeping flow [51]. Weeping flow can breakup nonwetting bubbles or droplets by inducing gradients in canillary pressure along their surfaces. An example of a weeping-flow breakup mechanism 


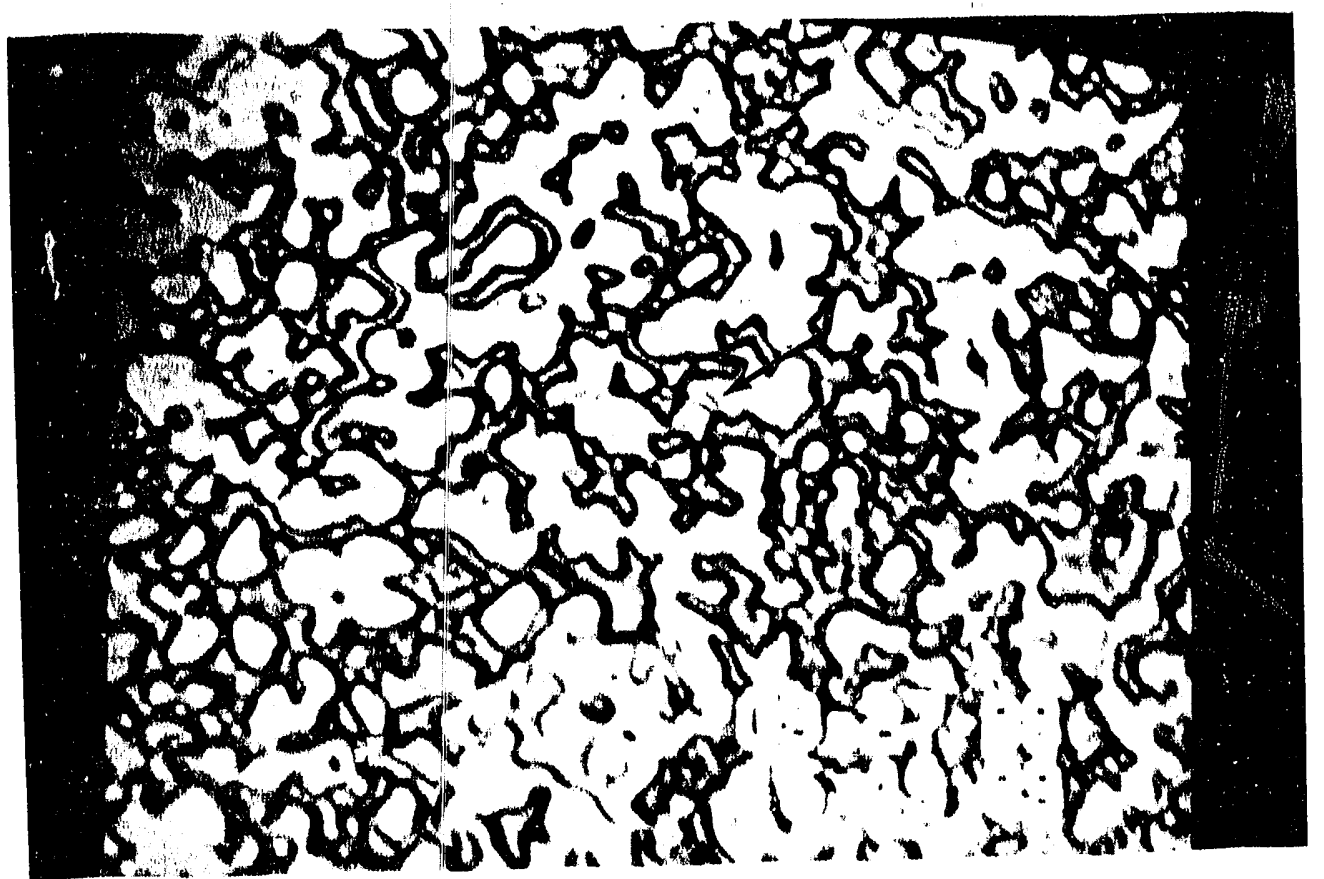

(a)

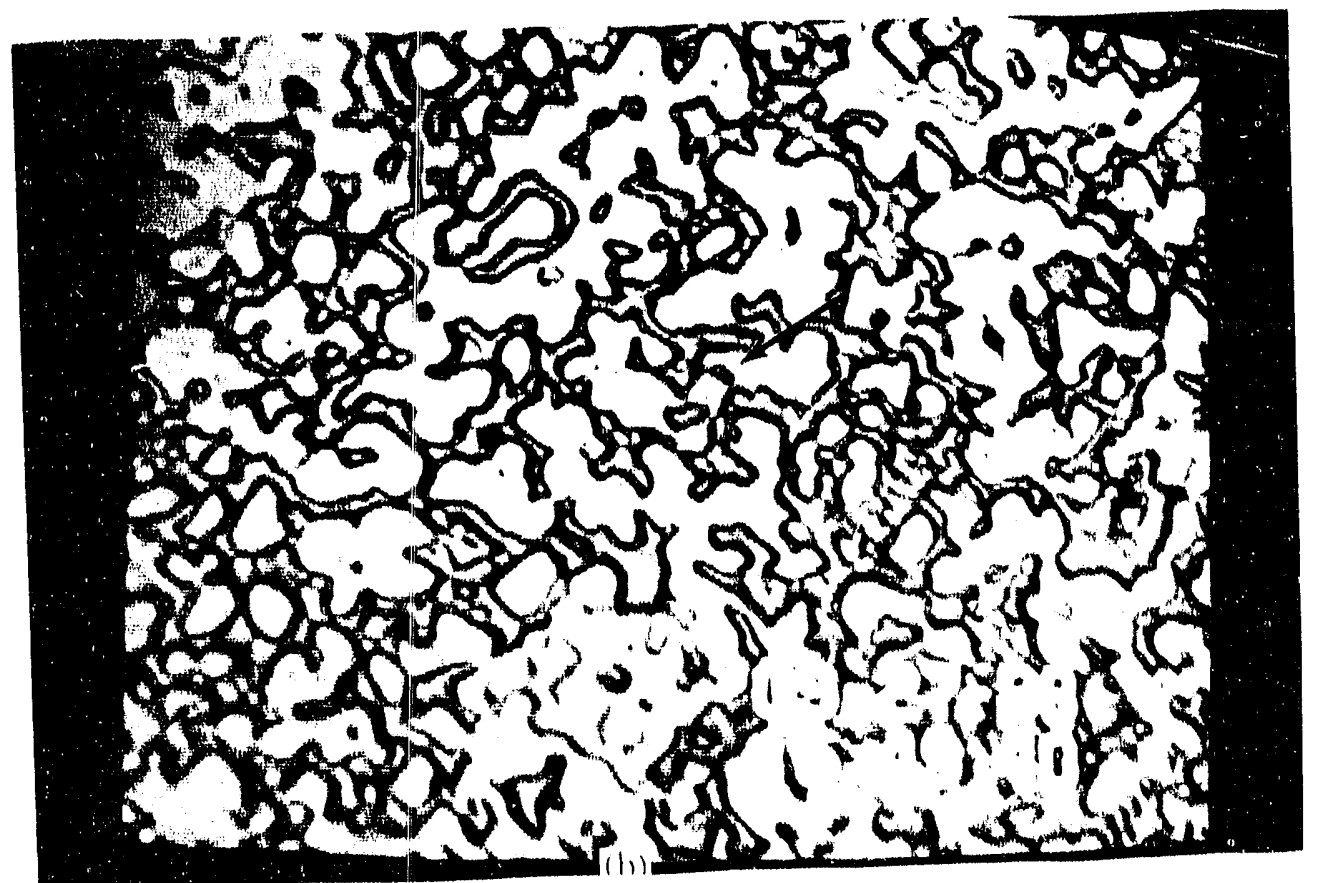

Figure 17: Microphotographs of an intermittent snap-off event recorded while injecting an $80 \%$ quality foam comprised of air and surfactant solution at a total superficial velocity of $62 \mathrm{~m} /$ day: (a) liquid (surfactant solution) occupies pore throat highlighted with an arrow, (b) gas enters throat form left and displaces liquid, 


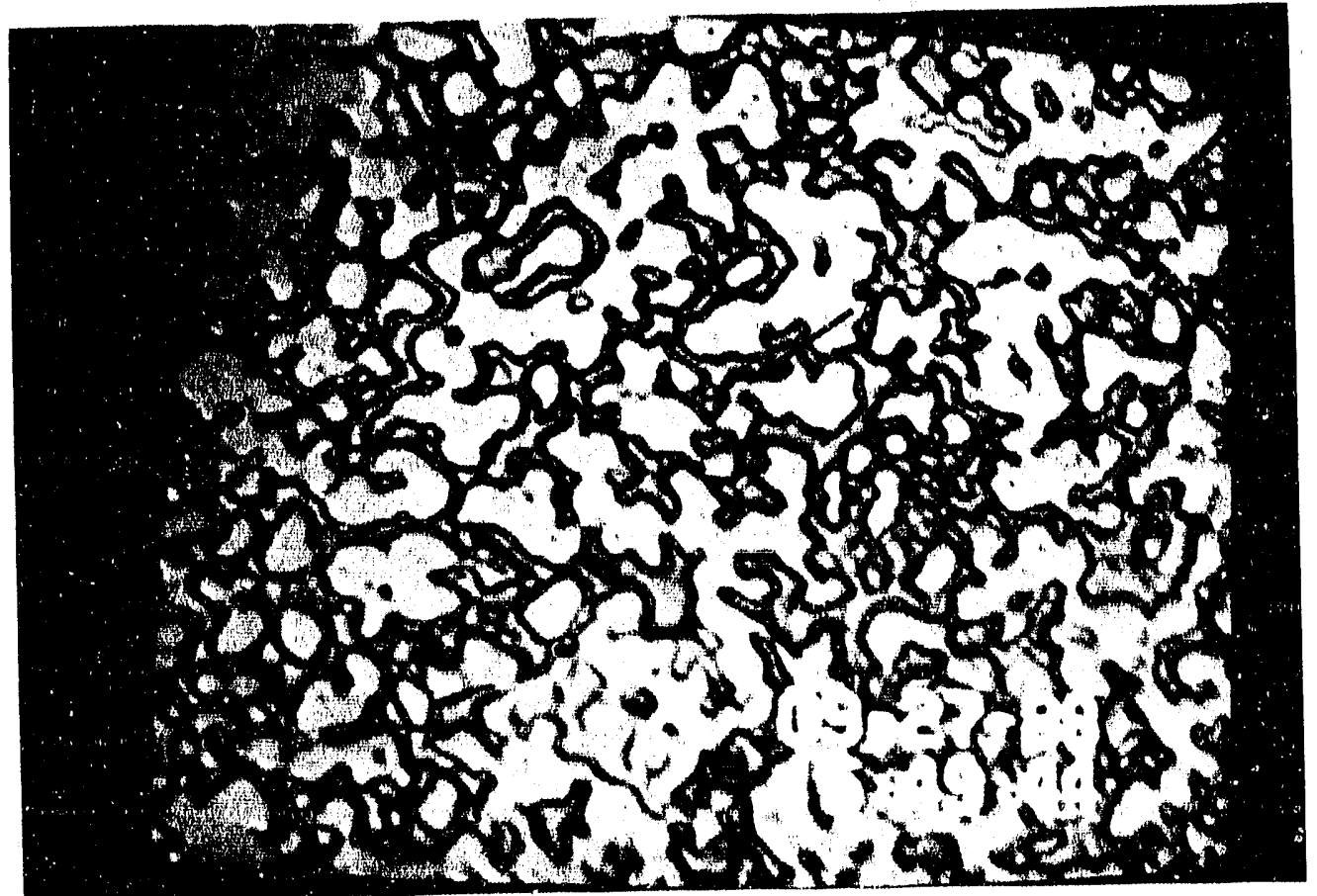

(c)

Figure 17: (c) liquid imbibes into pore throat 4 seconds later (note date and time (cont.) information in lower right) and snaps-off a bubble as a result of a local capillary-pressure fluctuation. 
appears in Figure 18. The microphotographs in this figure were taken while injecting 0.5 wt \% active surfactant solution without any gas into the event micromodel at a superficial velocity of $13 \mathrm{~m} /$ day following a foam-flood experiment. The overall direction of flow is from the top to the bottom of the photographs in this breakup sequence. A gas bubble is just en iering a pore throat in the center of the photograph in Figure 18a. The throat is highlighted with an arrow. Figure $18 \mathrm{~b}$, recorded $1 / 30$ of a second later, shows a small bubble snapping-off in the pore throat and quickly accelerating into the attached pore body. The dim outline connecting this small gas bubble, which is located at the inlet to the large pore body appearing just above the date and time information in Figure 18b, with the miminum constriction in the attached pore throat is a consequence of the high bubble velocity and limited time resolution of the video recording equipment (1/30 of a second) rather than an indication of a connected gas thread.

The snap-off mechanism illustrated in Figure 18 occurs as surfactant solution, flowing around the bubble trapped against the constriction, induces a large gradient in capillary pressure near the front of the bubble. The gradient in capillary pressure subsequently modifies the curvature along the gas-liquid interface. Snap-off occurs when the curvature of the interface just upstream of the pore constriction reaches the critical value depicted in Figure 11b. Ransohoff and Radke [51] first explained this phenomenon in their treatment of weeping flow around a trapped gas bubble. The diameter of a foam bubble created by this snap-off mechanism is of the order of the pore-throat diameter rather than the pore-body diameter, as shown by the small size of the bubbles snapped-off in the large, mostly-liquid-occupied pore body appearing just above the date and time information in Figure 18. It is not possible to locate unequivocally the snap-off position in this figure due to the constraints in resolution imposed by the video recording equipment, but it does appear that snap-off occurred very near to the mimimum in the pore constriction. Figure

$18 \mathrm{c}$ indicates that weeping flow continues to operate in this pore throat resulting in the formation of many small gas bubbles from the original train of relatively larger bubbles. 


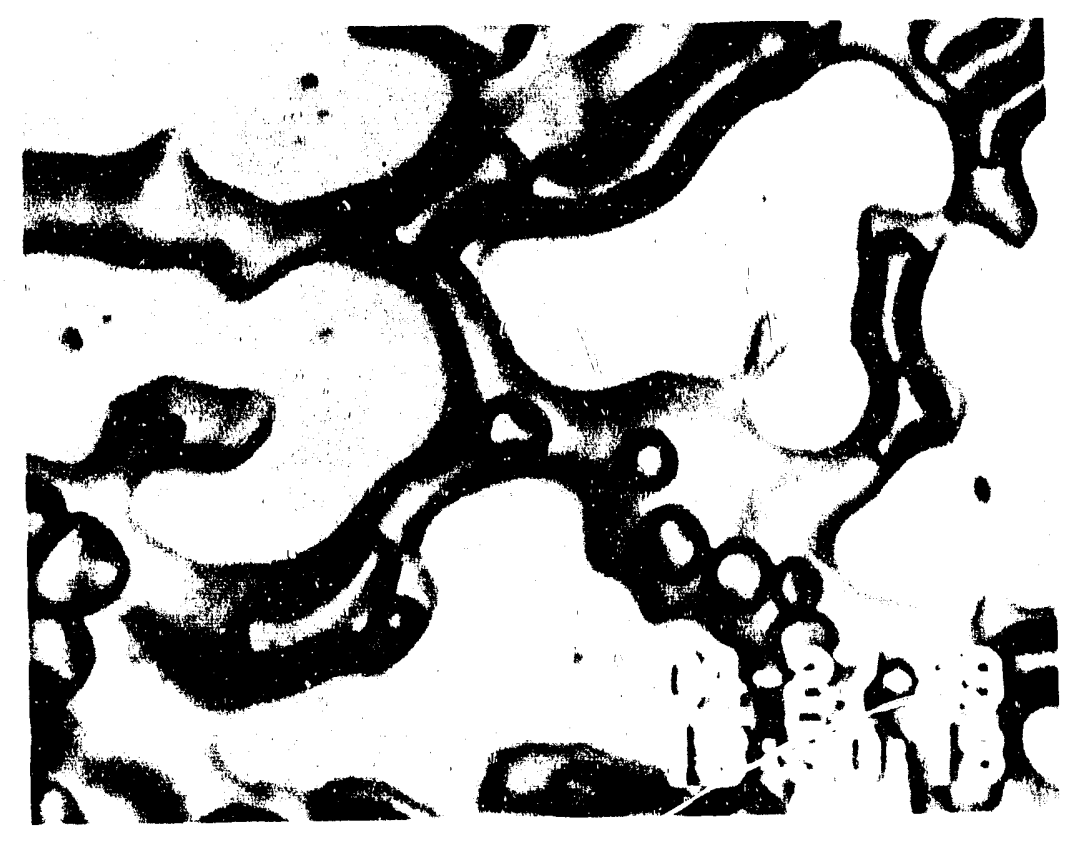

(a)

Figure 18: Microphotographs of weeping flow causing snap-off upstream of a pore throat recorded while injecting wetting liquid (surfactant solution) at a superficia! yelocity of $13 \mathrm{~m} / \mathrm{day}$ following a foam flood: (a) liquid flowing from top to bottom forces a gas bubble into a pore throat (nighilghied with an arrow), 

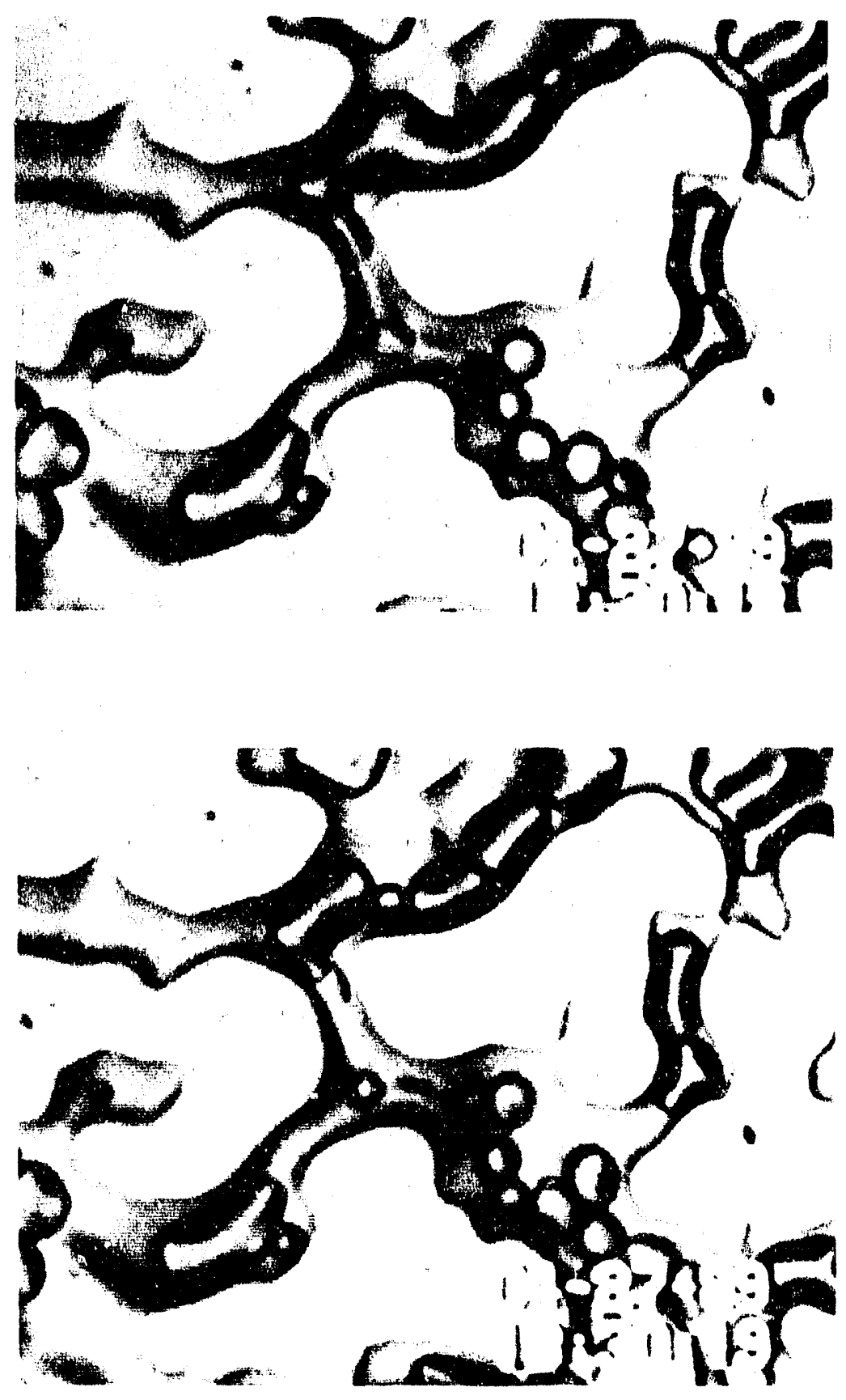

(c)

Figure 18: (b) microphotograph recorded 1/30 of a second later indicates that a bubble (cont.) snaps-off at or slightly upstream of the minimum constriction in the throat (the dim outline running the length of the pore throat and into the large pore body located just above the date and time information is a consequence of the high bubble velocity and limited time resolution of the video equipment (1/30 of a second)), (c) snap-off repeats due to weeping tlow in the same pore throat resulting in the formation of bubbles with radii on the order of the pore-throat size. 
Arriola et al. [52] also noted this phenomenon when injecting water into a single constricted square capillary containing a nonwetting oil drop trapped against the constriction. Ratulowski and Chang [44] performed a linear stability analysis, valid once the axisymmetric configuration shown in Figure $11 \mathrm{~b}$ is reached, in an attempt to estimate the size and frequency of bubble formation by this mechanism.

An important result of the weeping-flow behavior discussed above is that snap-off only occurs over a narrow range of flow conditions for a given pore-throat-body site. If the aspect ratio of the pore body to pore throat is not sufficiently large, then the wetting liquid forces the trapped gas bubble through the constriction without breaking it up [34, 51]. As discussed earlier, Legait [34] used the lubrication approximation to the NavierStokes equations to solve the hydrodynamics for creeping, two-phase flow in constricted square capillaries in order to quantify the mobilization criteria for trapped nonwetting drops. His calculations are in good agreement with previous estimates for mobilizing oil droplets in porous media [53].

Huh et al. [12] noted breakup of a nonwetting $\mathrm{CO}_{2}$ thread in a micromodel study when injecting surfactant solution into a region swept previously by $\mathrm{CO}_{2}$ flooding. They observed, however, that a large aspect ratio is not necessary for breakup to occur under these flow conditions. Furthermore, they noted that breakup occurred along the preferential flow path in their model and that the sizes of the bubbles created were larger than those created by the drainback mechanism (see Figure 6 in ref. [12]). Weeping-flow breakup can also explain this behavior. Following a gas injection experiment, the gas saturation and capillary pressure are relatively high in the micromodel. As a result, the gasliquid interface along the flow paths filled with foam resembles the interface in Figure

11a. Weeping flow reduces curvatures along the long gas thread just as it modifies curvatures along a short trapped bubble. Breakup of the thread occurs at those points where weeping flow results in the unstable configuration shown in Figure $11 \mathrm{~b}$. Once the gas thread reaches this unstable configuration, the breakup process proceeds by the same 
mechanism observed by Aul and Olbricht [43] in their pressure-driven, core-annular flow experiments. A direct consequence of this behavior is that breakup will occur at periodic intervals of approximately $23 / 2 \pi R$ where $R$ is the radius at some location along the flow channel. Eqn. ( 4) indicates that smaller pores have higher values for the critical curvature for breakup. Consequently, breakup is expected to occur at pore constrictions first because they cannot withstand reductions in curvature as well as larger pores.

The microphotographs in Figure 19, taken at the same flow conditions as Figure 18 , illustrate the breakup of a gas bubble in a pore throat due to weeping flow. A long gas bubble is driven up against a pore constriction, highlighted with an arrow, by wetting liquid in the lower left of Figure 19a. The bubble is driven into the constriction in Figure

19b. The gas thread breaks away from the wall into an unstable axisymmetric thread in Figure 19c. Breakup occurs less than 1/30 of a second later, as evidenced by the appearance of a relatively large, circular (in plan view) gas, bubble directiy above the " 0 " in the date information in Figure 19d. Breakup occurs in the tightest part of the constriction, as predicted. Note that the four gas bubbles formed by this mechanism in the large pore body located at the lower right of Figure $19 \mathrm{~d}$ are significantly larger than those formed by upstream breakup in the same pore body in Figure 18 . The curvature at the front of the gas bubble in Figure $19 \mathrm{c}$ is very similar to the curvature at the front of the bubble in Figure $14 \mathrm{c}$ just prior to snap-off indicating that the same mechanism of liquid drain back might be at work here. Although the curvature at the front of the gas bubble in Figure $19 \mathrm{c}$ is clearly lower than that in the constriction, snap-off did not occur at this site during the initial foam flood which was performed in the drainage mode of injection. In other words, weeping flow was required to initiate breakup (snap-off) because this site apparently does not meet the geometric constraint for snap-off.

The gas bubbles associated with weeping-flow breakup in Figure 19 do not appear to have diameters of $23 / 2 \pi R_{l}$ where $R_{l}$ is the radius of the pore constriction. instead they appear to have diameters of approximately $4 R_{b}$, which is somewhat smaller than 


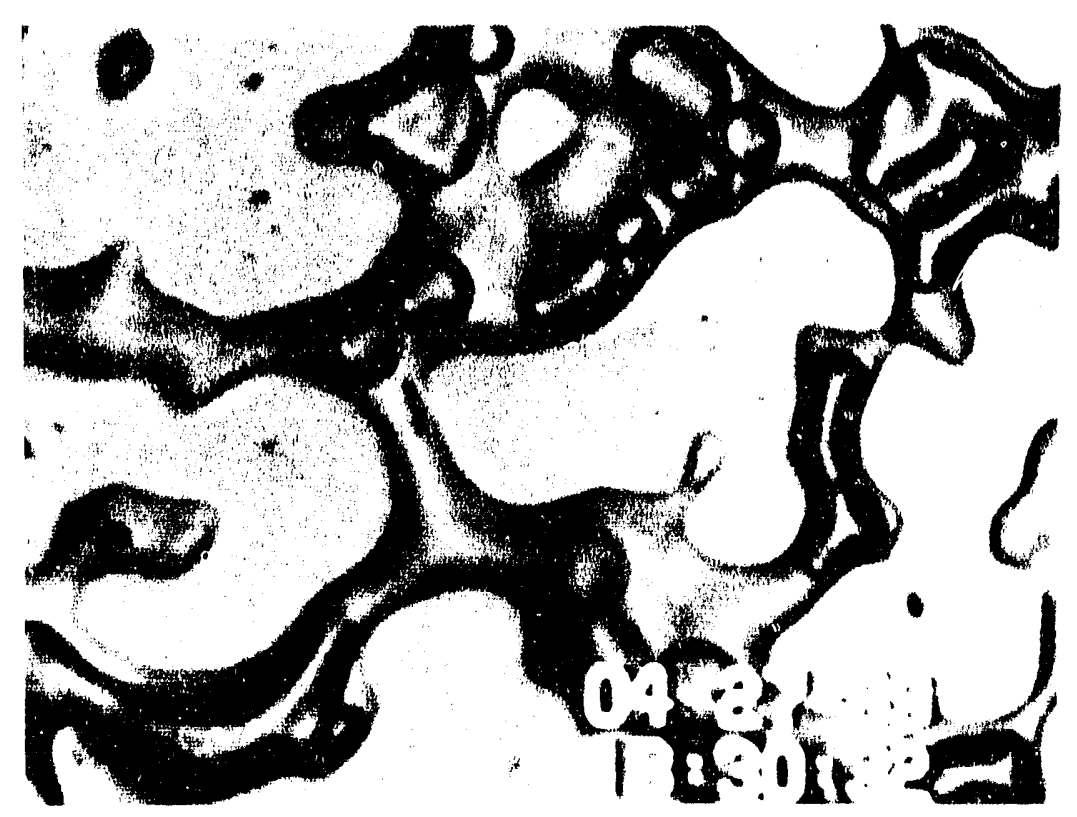

(a)

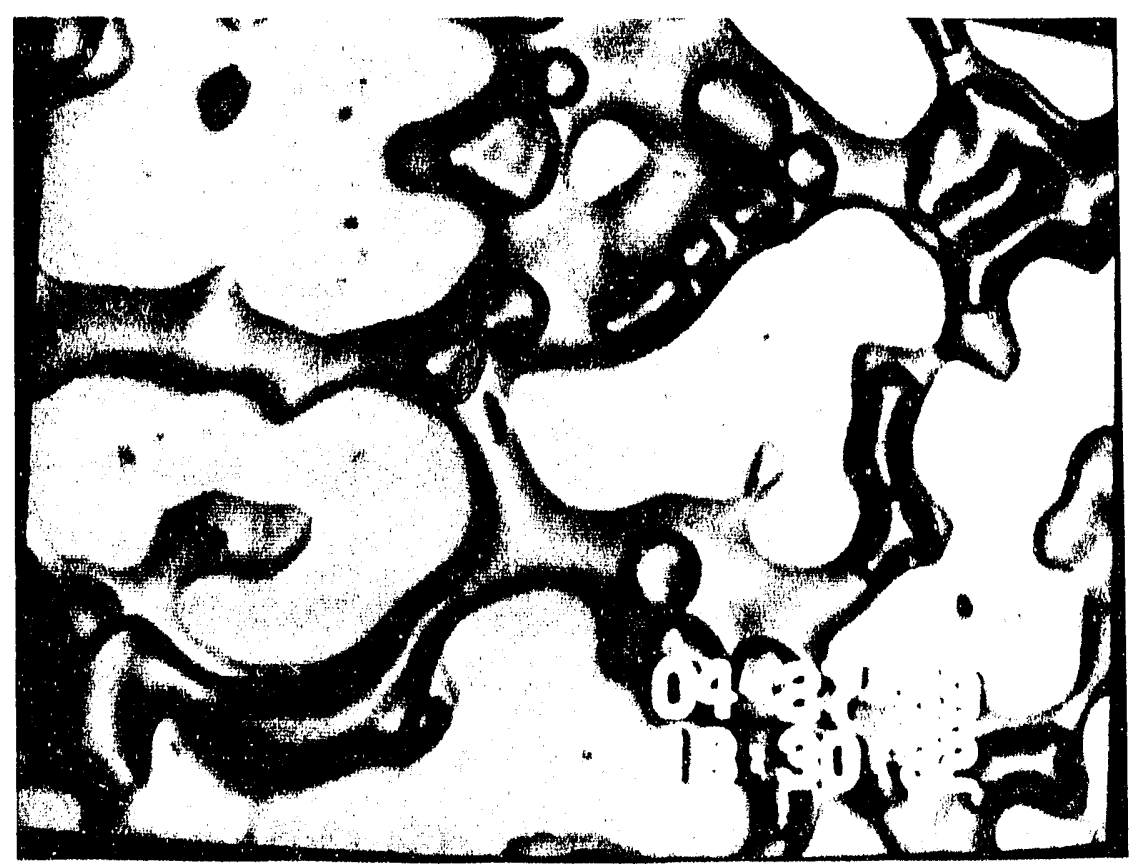

(b)

Figure 19: Microphotographs of weeping-flow breakup of a long bubble in a pore constriction at the same conditions as Figure 18: (a) long bubble is trapped against a pore constriction (highlighted with an arrow), (b) weitiig-liuguid flow forces bitbolc into constriction, 


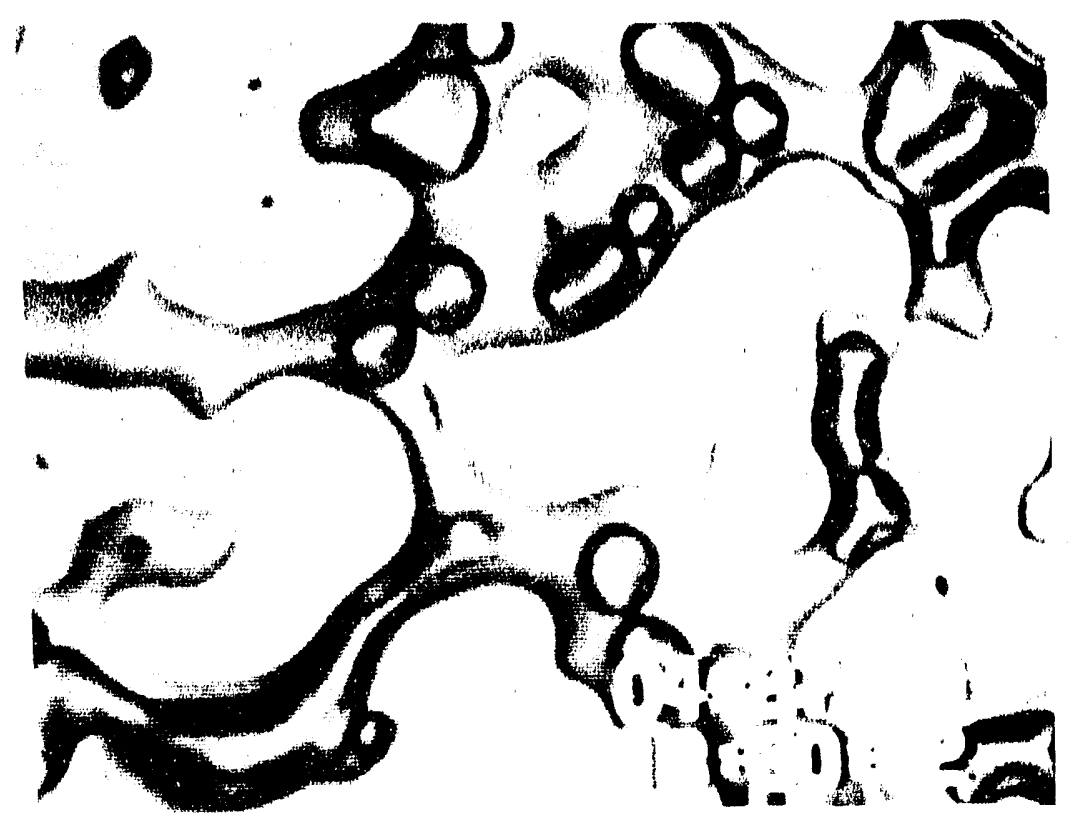

(c)

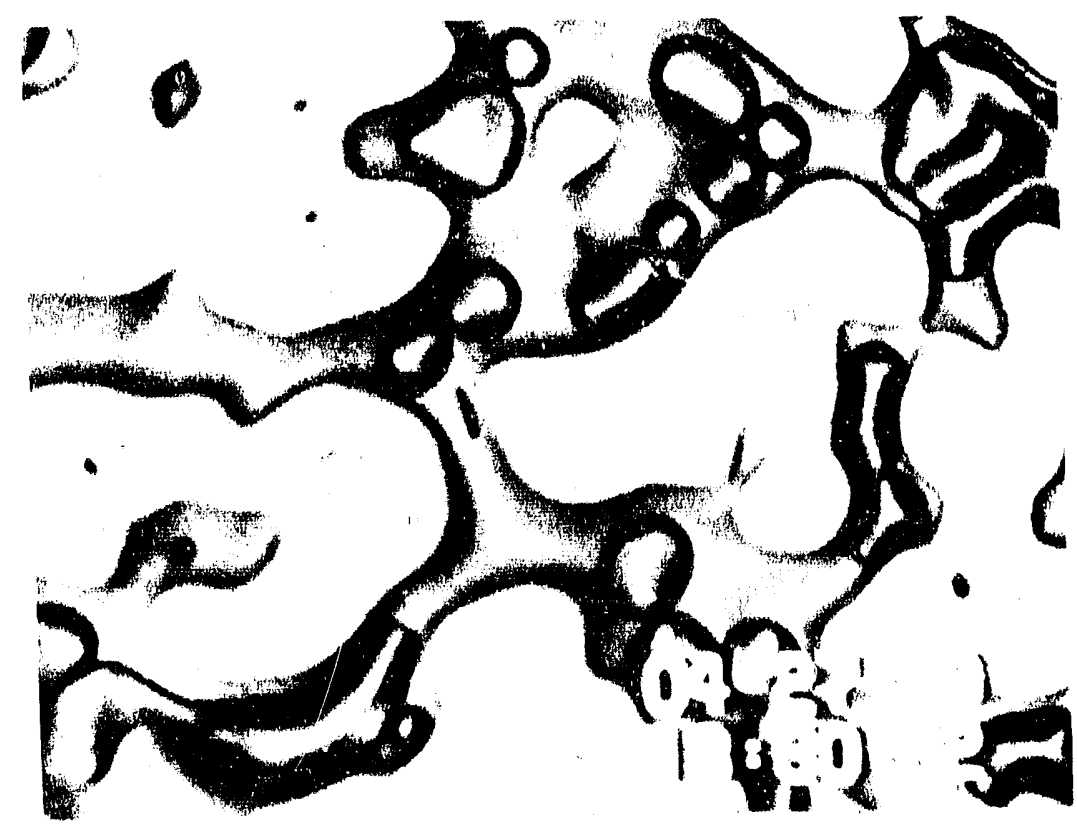

(d)

Figure 19: (c) gas breaks away from pore wall in constriction indicating the formation (cont.) of an unstable liquid collar, (d) breakup occurs less than 1/30 of a second látér. 
expected. Keep in mind, however, that the radii of the pores appearing in plan view in Figure 19 are probably not the controlling dimension in terms of capillary phenomena as a result of limitations in the micromodel fabrication process (see Section 7.3). Consequently, it is reasonable to expect that the capillary radius of the pore throat of interest in Figure 19 is somewhat smaller than the radius appearing in plan view resulting in smaller gas bubbles. Note that weeping flow has also driven the gas bubble into an apparently unstable axisymmetric configuration in the lower left of Figure 19, but breakup was not noted here. The explanation for the stability of the gas-liquid interface here is that the termination of the solid boundary on the lower side of the bubble, due to an intersecting flow channel, precludes the formation of the long wavelength disturbances that induce breakup.

\subsection{Lamella Division}

Once formed by snap-off or weeping-flow breakup the number of flowing lamellae increase by the mechanism of lamella division $[12,17,18,54,55]$. The microphotographs in Figure 20, taken while injecting air at a superficial velocity of $13 \mathrm{~m} /$ day into the event micromodel, which was initially saturated with $0.5 \mathrm{wt} \%$ active surfactant solution, illustrate the lamella-division process. The flowing foam bubble indicated with an arrow advances into a small pore body in Figures $20 \mathrm{a}$ and $\mathrm{b}$ and then splits into two separate bubbles as the lamella contacts the far pore wall in Figure $20 \mathrm{c}$. The newly created lamella then convects off from the division site in Figure. 20d. Division occurs as the lamella contacts the far pore wall in Figure $20 \mathrm{c}$ due to stability considerations. In general, a lamella is a surface of constant or nearly constant curvature. Furthermore, a $0^{\circ}$ contact angle must exist at the gas-liquid-solid boundary in a strongly water-wet medium, which describes the micromodels used in this work. A lamella also exhibits an apparent contact angle of $\hat{O}^{\circ}$ ât thic trañsituoun from the Plateati border into the thin-film portion of the liquid interface between two gas bubbles [56]. The above three requirements cannot be met by a 




(a).

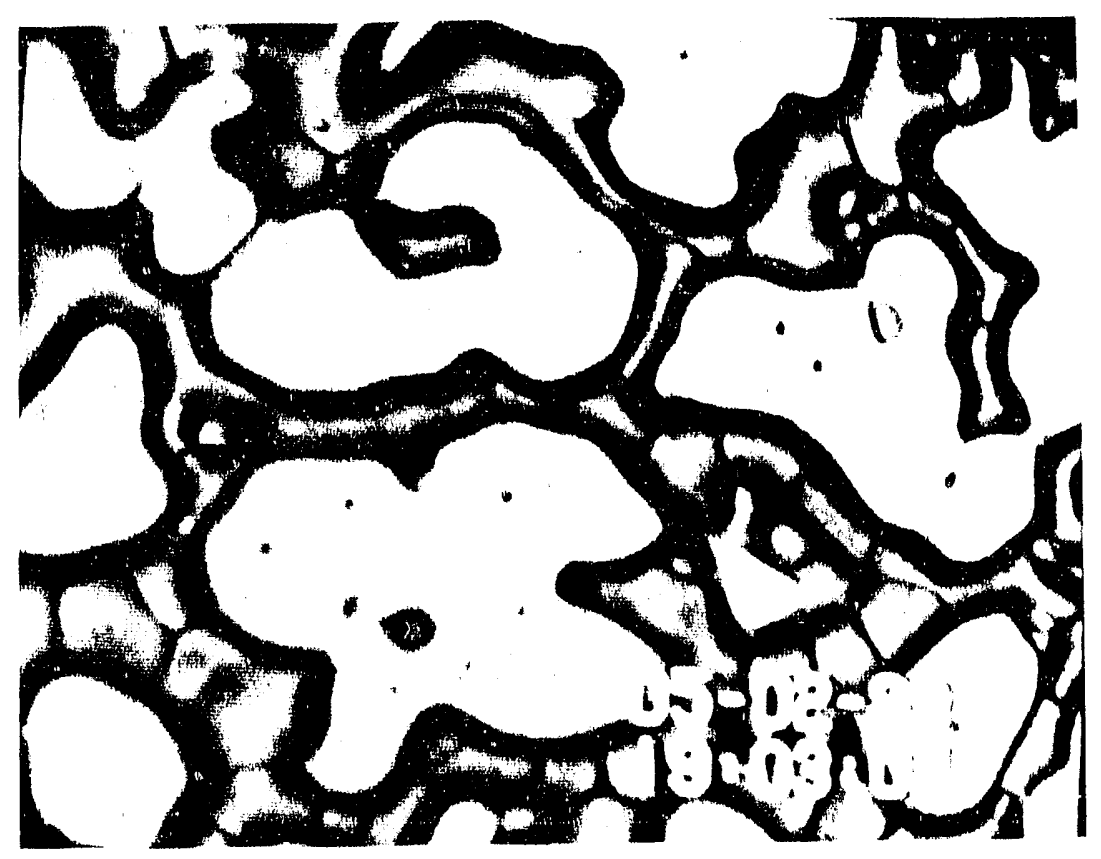

(b)

Figure 20: Microphotographs of lamella division at a $Y$-shaped pore junction during gas (air) injection at a superficial velocity of $13 \mathrm{~m} /$ day into an etched glass micromodel initially saturated with surfactant solution: (a) lamella (highlighted with an arrow) flows toward $\mathrm{Y}$-shaped junction, (b) lamella bows away from pore walls as it expands into junction, 
lamella in the configuration illustrated in Figure 20r as it first contacts the far po e wall, whereas they are met in the configuration illustrated in Figure 20d once the lamella divides.

Hirasaki [55] speculated that lamella division is the predominant mechanism for generating lamellae in the presence of a large pressure gradient. The two requirements for lamella division are mobile lamellae and branching in the foam flow paths. As noted earlier, experimental observations [3,57-59], arguments for pressure mobilization of lamellae trains [10], and extensions of EMT calculations indicate that foam only flows through a small portion of the pore space. However,

a significant amount of branching occurs in these flow paths. The constantly changing flow paths observed during micromodel studies of foam flow $[17,18]$ are another source of lamella division.

An example of lamella division due to the intermittent mobilization of a trapped foam cluster appears in Figure 21. The experimental conditions for this figure are the same as those for Figure 20. Lamellae trains flow continuously through the lower branch of the pore, which is highlighted with an arrow in the left-center of Figure $21 \mathrm{a}$, without dividing while the lamellae in the upper branch remain essentially stationary. However, they appear to "breathe" in response to local pressure fluctuations [60]. The short lamellae train in the upper branch begins to flow in Figure $21 \mathrm{~b}$ although this is difficult to discern in this sequence of still microphotographs. A large foam bubble, indicated with an arrow for a lamella that spans the entire pore body of interest, enters from the left in Figure 21c as foam continues to flow in both the upper and lower channels. This lamella divides at the outlet of the pore body in Figure $21 \mathrm{~d}$ and the two lamellae, both highlighted with arrows, convect away from this division site. Foam becomes trapped once again in the upper channel soon after division occurs 


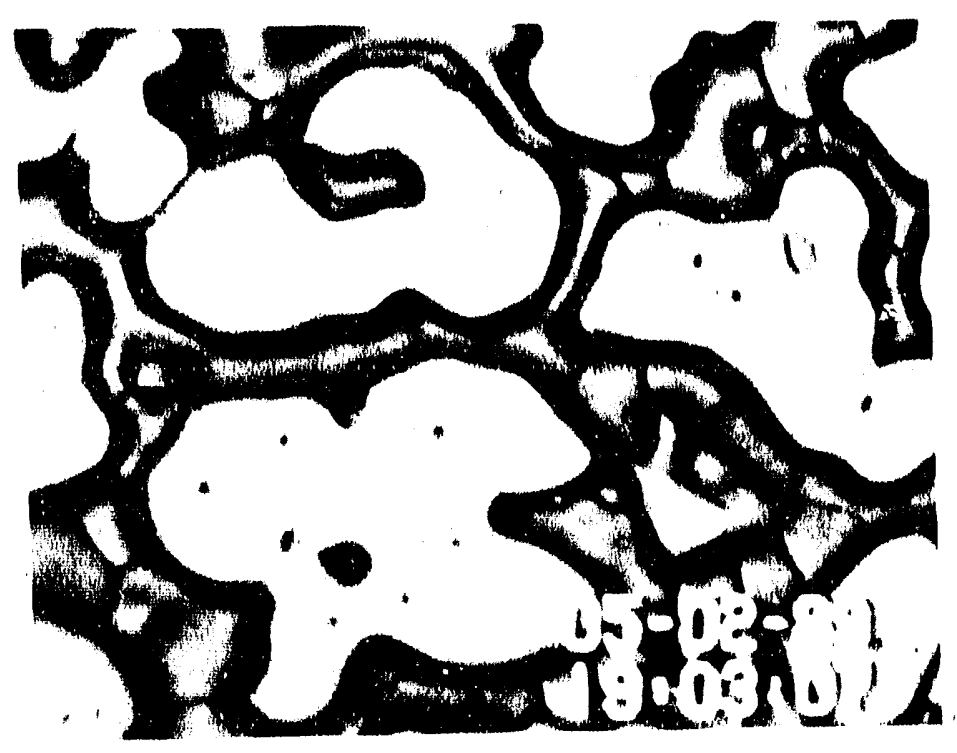

(c)

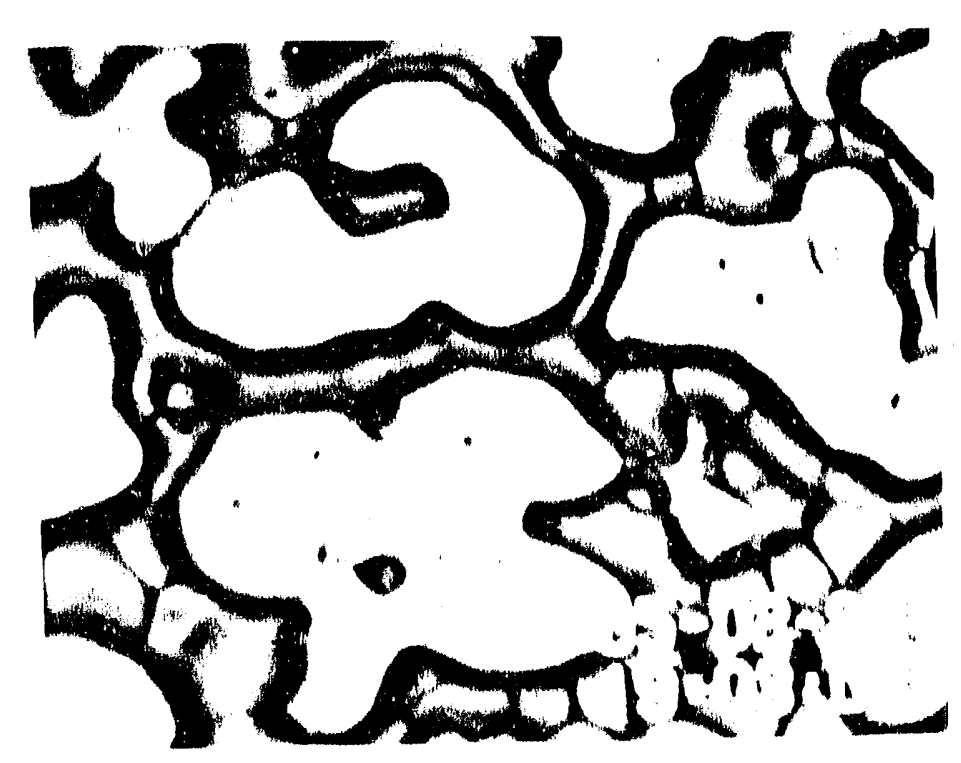

(d)

Figure 20: (c) lamella continues to bow as it expands farther into junction (cont.) (note apparent contact angle of 900 with pore walls), (d) 1.amelita d:tvides upon contact with far pore wall. 




(a)

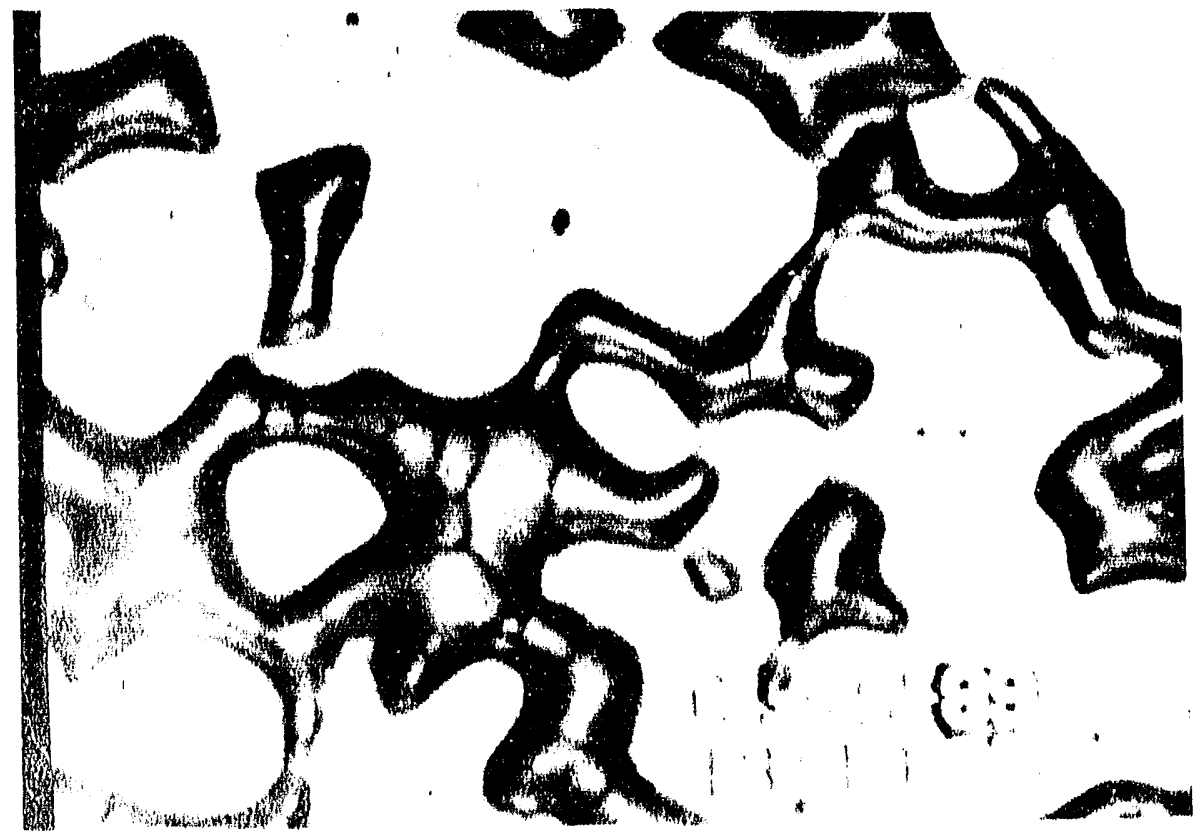

(b)

Figure 21: Microphotographs of intermittent lamella division at the same conditions as Figure 2,20: (a) lower flow branch leading from pore body at left center of microphotograph contains foam that flows from left to right while upper branch contains trapped foam, (b) foum is mobilized in upper branch, 


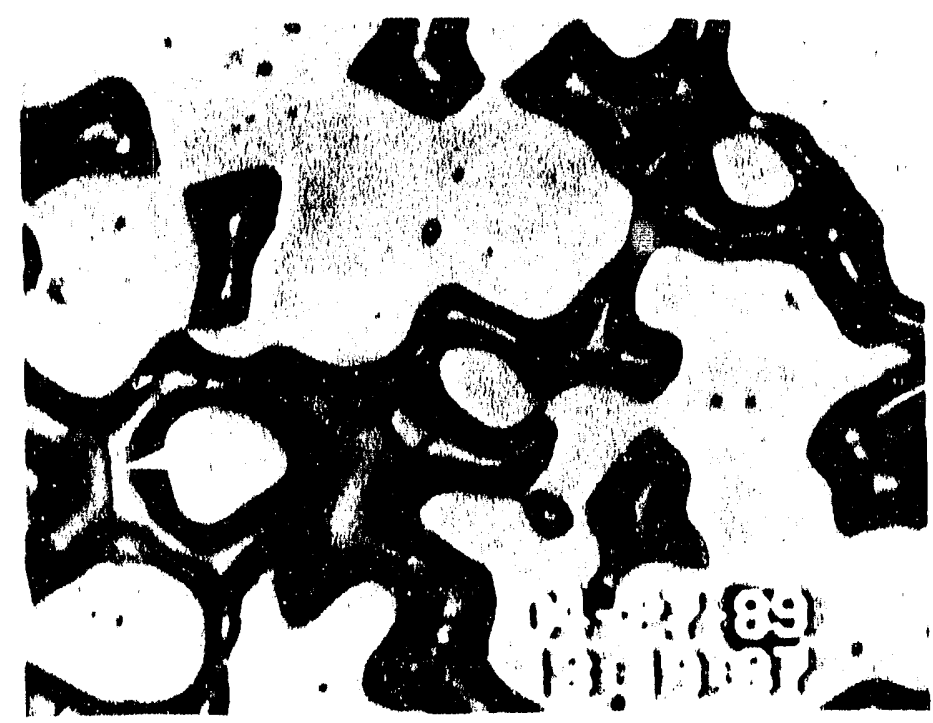

(c)

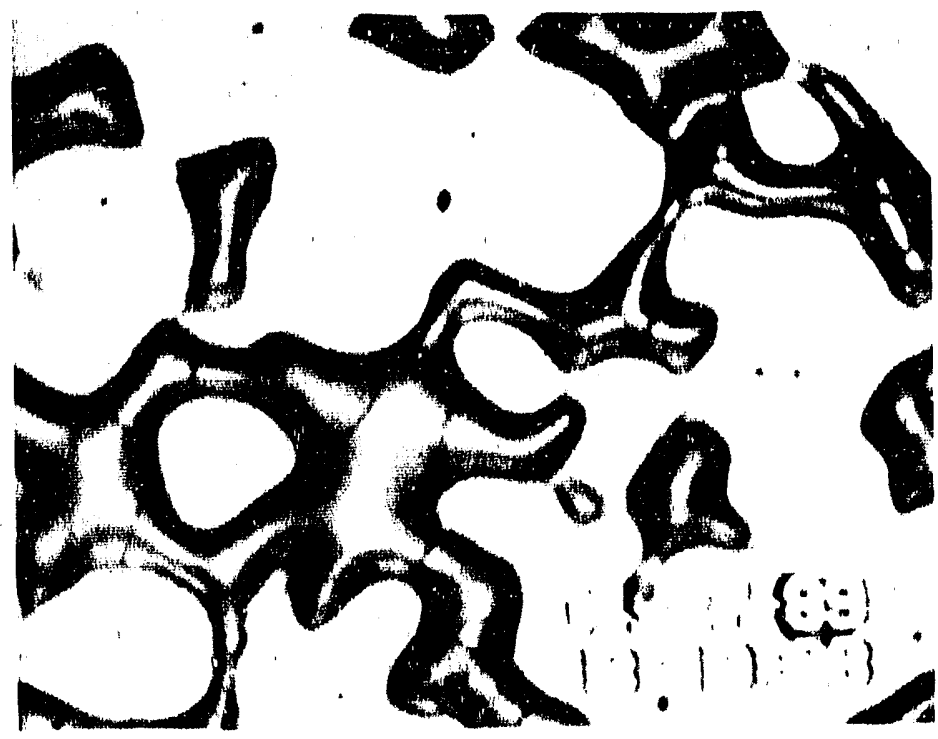

(d)

Figure 21: (c) large foam bubble with a lamella (highlighted with an arrow) spanning (cont.) the entire pore body convects toward outlet of body, (d) lamella divides at outlel of pore body. 
Another important observation of lamella division is that large gas bubbles have a greater tendency to divide than smaller ones. Figure 22, also recorded at the same experimental conditions as Figure 20, illustrates this tendency. In contrast to the behavior of the large gas bubble shown in Figure 21, the small gas bubbles located in the large pore at the left-center of Figure 22a do not divide as they flow through this branching point in the foam flow path. Instead some flow through the upper branch and some through the lower branch, as shown in Figures $22 \mathrm{~b}$ and $\mathrm{c}$. A large foam bubble, indicated with an arrow by a lamella that spans a large pore body, enters from the left in Figure 22c. This large foam bubble does divide, however, as shown by the newly created lamella that is highlighted with an arrow in Figure $22 \mathrm{~d}$.

\subsection{Lamella Leave-Behind}

Figure 23 illustrates the lamella leave-behind process [54-56]. This sequence of microphotographs was recorded while injecting an $80 \%$ quality foam comprised of air and 0.005 wt. \% active surfactant solution at a total superficial velocity of $31 \mathrm{~m} /$ day. As shown in Figures 23a and b, lamella leave-behind occurs when two gas fronts converge on the same liquid-filled pore space from different directions. A lamella forms in Figure 23c and $\mathrm{d}$ as the converging gas fronts drive the liquid out of the pore space betweer. them. Note that the area of interest has moved toward the center of the microphotograph due to repositioning the camera. As long as surfactant is present to stabilize the interface and the capillary pressure in the medium is not too high (see Section 10), then a stable stationary lamella results. The leave-behind process does not require that two gas fingers converge simultaneously on the same liquid-filled region. Rather a gas finger arriving at a later time through a liquid-filled region can converge on an existing gas-filled channel and squeeze down the lamella as the canillary pressure increases. .

Lamellae created by leave-behind block gas flow channels thereby reducing the relative permeability to the gas phasc. The gas phase remains continuous, however, 


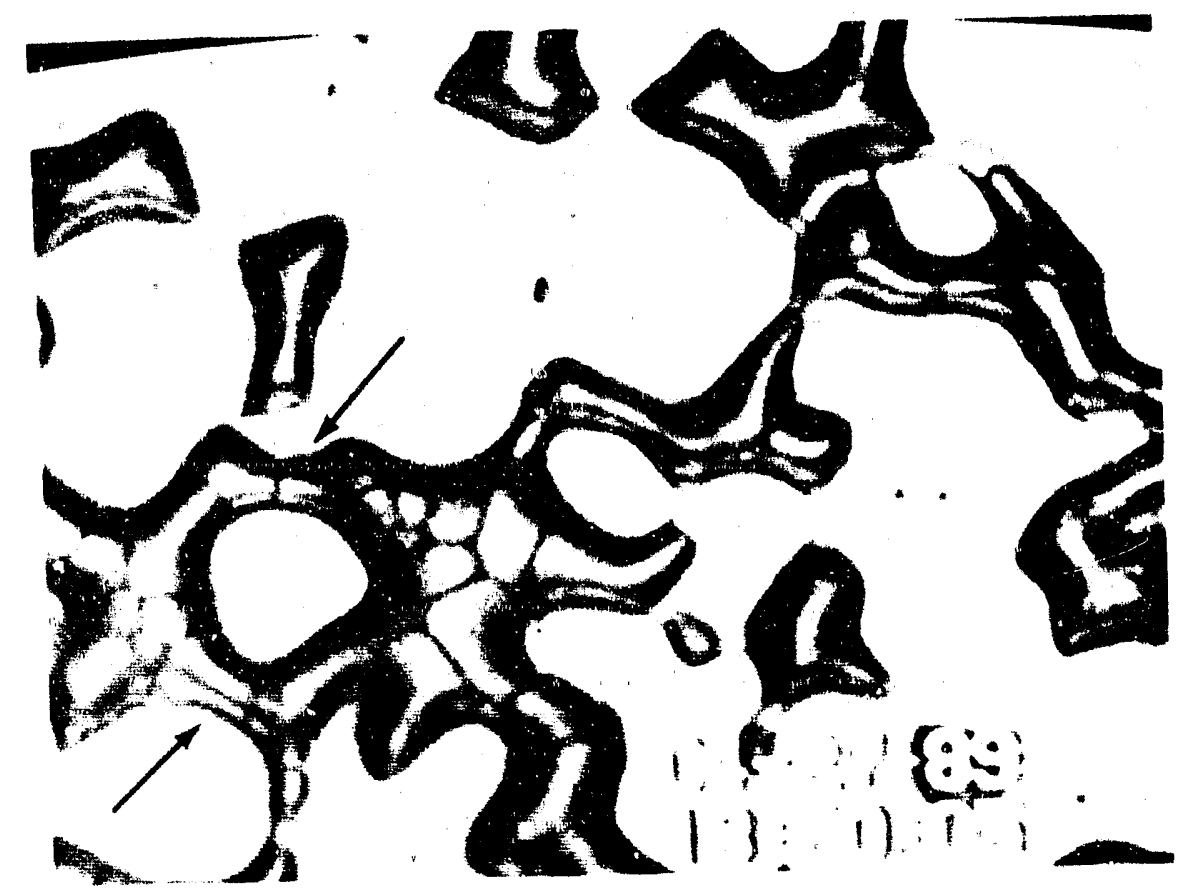

(a)

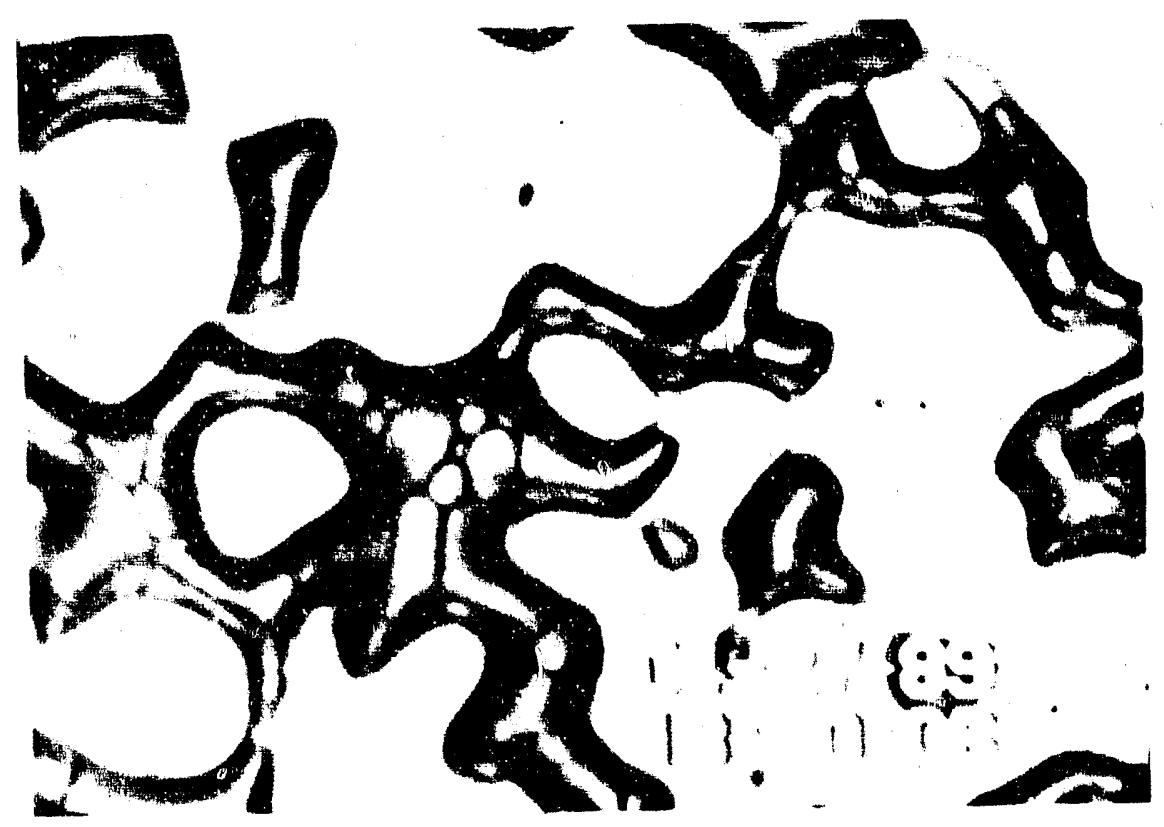

(b)

Figure 22: Microphotogranhs illustrating that small foam bubties do not readily divide: (a) foam flows in both upper and lower branches through the same site and at the same conditions discussed in Figure 21, (b) small foam bubbles separate at the downstream side of the pore body and flow out of it without dividing, 



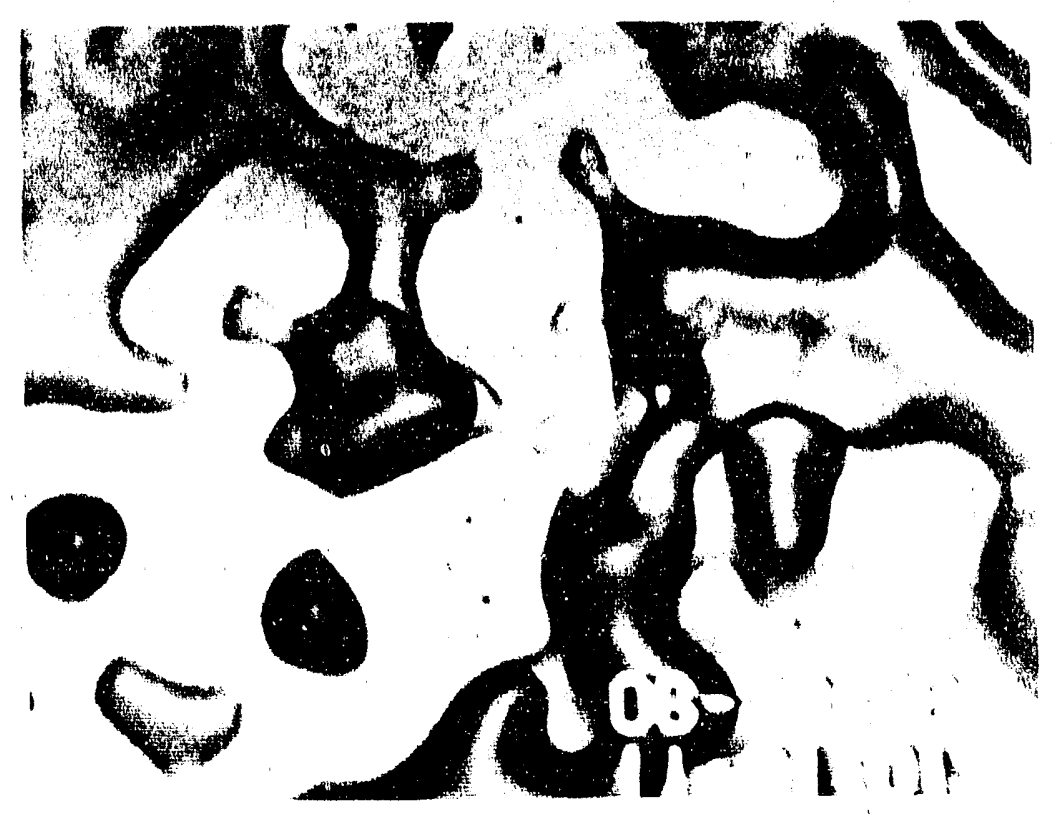

(a)

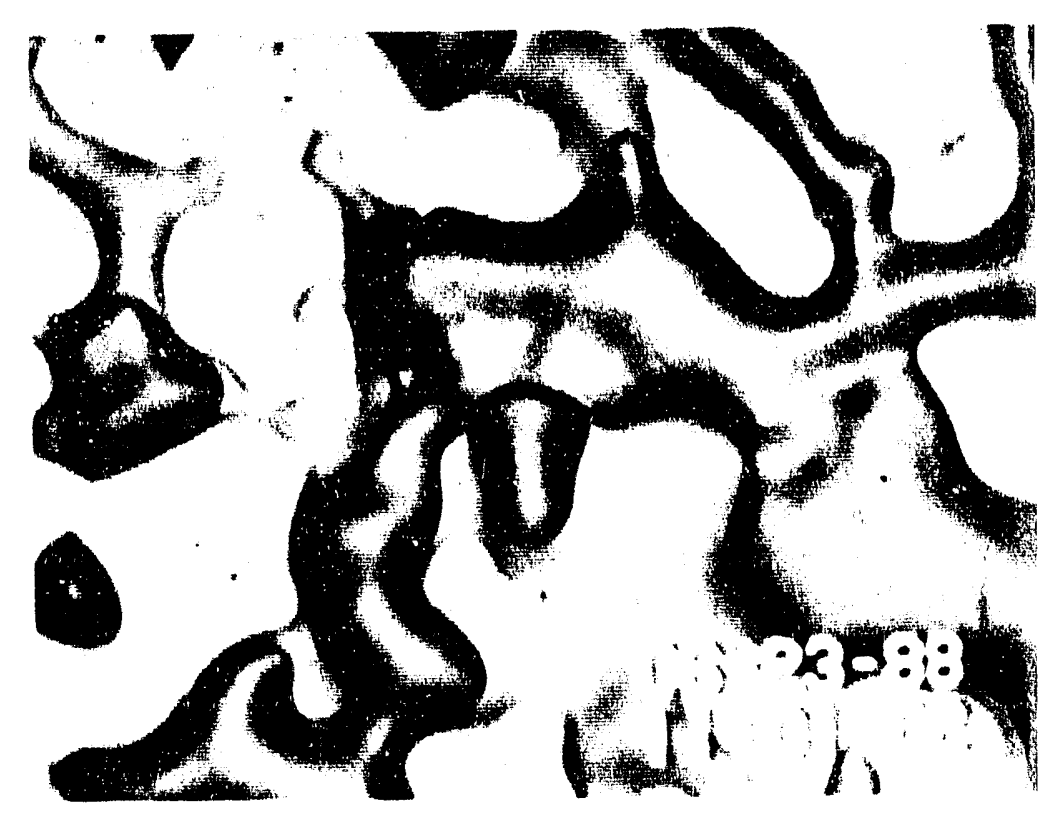

(b)

Figure 23: Microphontographs of lamella leave-behind recorded while injecting an $80 \%$ quality foam comprised of air and surfactant solution at a total superficial velocity of $31 \mathrm{~m} / \mathrm{day}$ : (a) two gas fingers in the upper right converge on the same surfactant-saturated region, (b) slower gas finger still converging, 


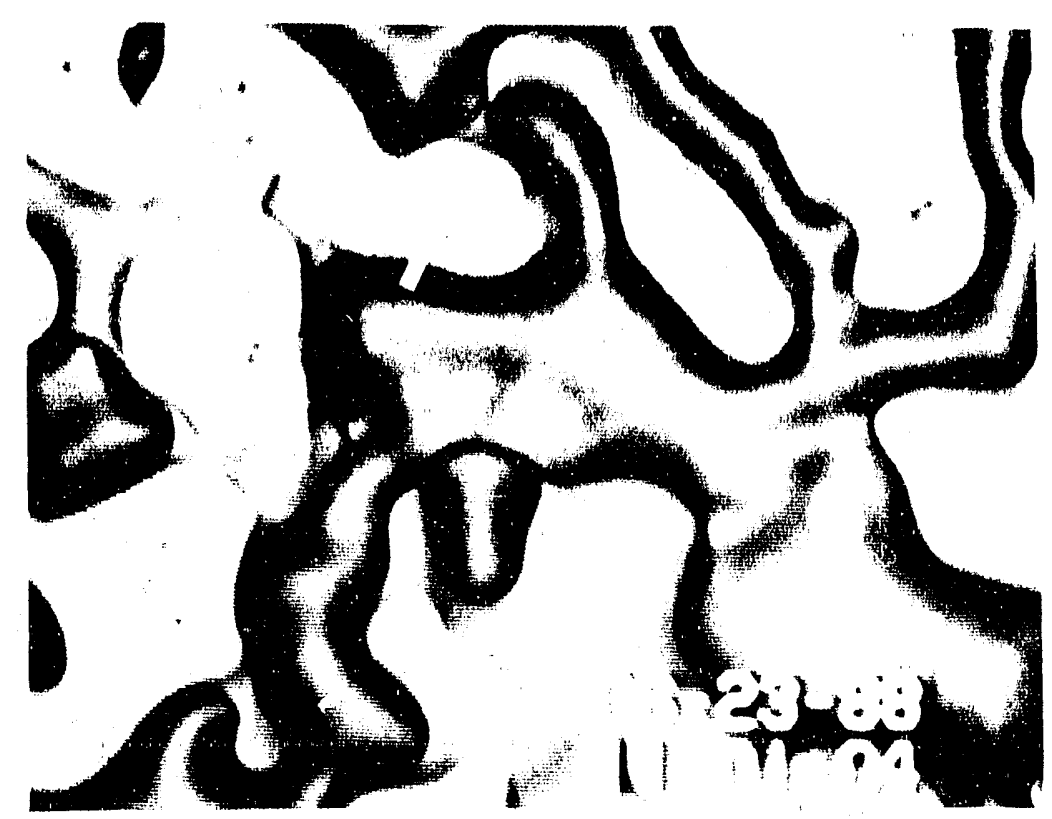

(c)

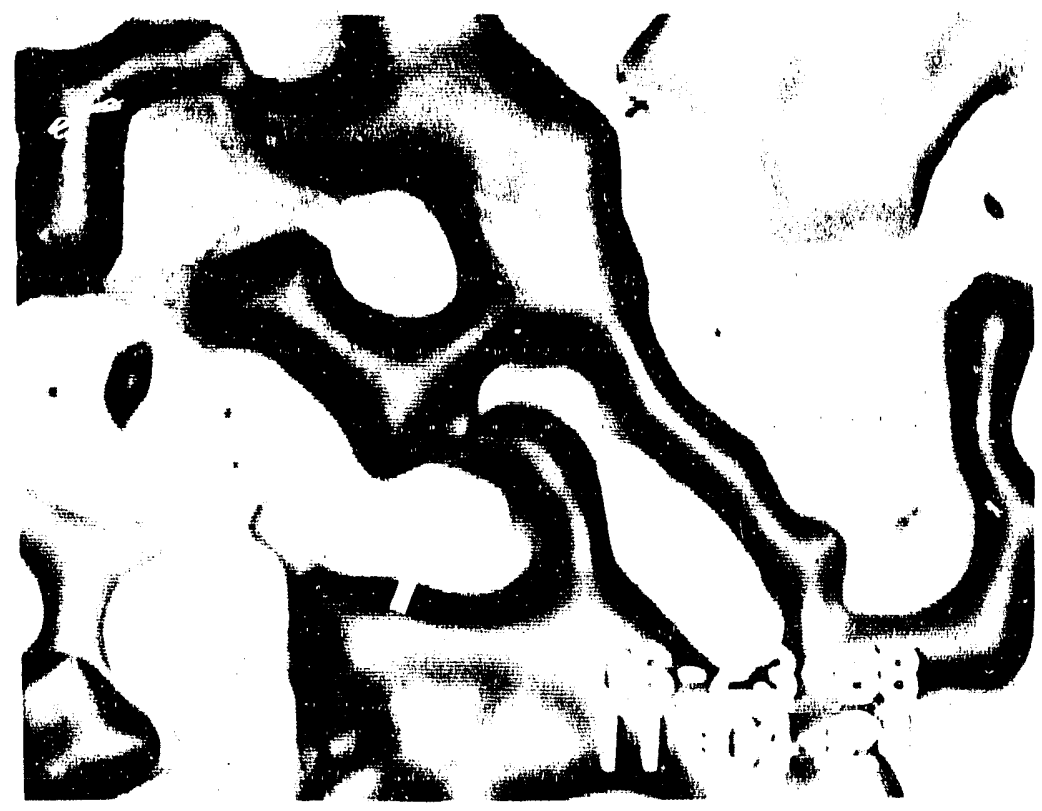

(d)

Figure 23: (c) gas converges to form a lens, (d) liquid has drained out of lens leaving a (cont.) lamella that opens up into Plateau borders (note that the area of interest has shifted to the center of the page due to repositioning the camera). 
because no discrete flowing foam bubbles are created. Data from experiments by Ransohoff and Radke [54] in beadpacks indicate approximately a five-fold reduction in the steady-state relative permeability to gas as the result of the leave-behind mechanism. Friedmann and Jensen [61] reported approximately an order of magnitude decrease in the relative permeability of gas during constant-pressure gas injection experiments in a Berea core as a result of this same phenomenon. Unless ruptured by a further increase in the capillary pressure $[62,63]$ or mobilized by an increase in the pressure gradient $[10,60]$, the leave-behind lamellae remain at their point of generation in the porous medium. If a leave-behind lamella does vacate a generation site, substantial liquid must reinvade this region before a new leave-behind lamella can be generated.

\section{Coalescence Mechanisms}

\subsection{Capillary-Pressure Considerations}

Foam coalescence mechanisms are as important as the generation mechanisms described above in predicting foam flow behavior in porous media.

Foam is thermodynamically unstable with respect to collapse into constitutive bulk phases due to the additional free energy associated with its large surface area. Nevertheless, some surfactant solutions give rise to foams that consist of nonthinning liquid films and thus are resistant to collapse. Ross [64] refers to such foams as metastable. A metastable foam is created by introducing a surfactant into the liquid phase of the foam. Surfactants stabilize foum by retarding the forces that act to thin lamellae.

The Derjaguin-Landau-Verwey-Overbeek (DLVO) theory of classic colloid science explains the origin and importance of the retarding force (pressure) in terms of attractive and repulsive molecular forces. In general, the magnitude of the retarding pressure, which is referred to as the disjoining pressure, $\Pi$, is a function of the film thickness $h$ and the chemistry of the system [40]. A typical disjoining-pressure isotherm appears in Figure 
24. The very steep rise in the disjoining pressure at small film thicknesses corresponds to structural forces, which are operative at molecular separation distances, while the maximum in the isotherm results from a combination of attractive van der Waals, indicated by the dashed curve $\Pi_{A}$, and repulsive electric double layer overlap forces, indicated by the dashed curve $\Pi_{R}$, which are operative at separation distances $\| p$ to approximately $10^{-7} \mathrm{~m}$. An important characteristic of the disjoining-pressure isotherm is that not all film thicknesses are metastable. Virij [65] showed via thermodynamic analysis (i.e., a surfaceenergy minimization) that when $\partial \Gamma / \partial \mathrm{h}$ is greater than zero, the film is unstable. Separation distances between foam bubbles corresponding to molecular dimensions are not expected to occur in porous media. Consequently, a metastable foam only exists for lamellae thicknesses greater than the critical thickness $h_{\text {crit }}$ noted in Figure 24.

The importance of the chemical formulation of the surfactant solution on foam stability in porous media relates to the interaction between the capillary pressure in the medium and the disjoining pressure in the foam lamellae. The augmented Young-Laplace equation provides the starting point for relating the capillary and disjoining pressure $\Pi$ as follows:

$$
\Delta \mathrm{P}=2 \sigma \mathrm{C}_{\mathrm{m}}+\Pi(\mathrm{h})
$$

where $\Delta P$ is the pressure drop across the interface, $\sigma$ is the surface tension, $C_{m}$ is the mean curvature, and $\mathrm{h}$ is the film thickness. Figure 25 shows t:vo gas bubbles separated by a stable lamella and its adjoining Plateau borders. This configuration corresponds to the picture of foam trains in porous media. As noted earlier, the disjoining pressure only operates in thin films of thickness less than about $10^{-7} \mathrm{~m}$. Therefore, application of Eqn. ( 7 ) to the Plateau borders of Figure 25 where the separation distances are much larger than $10^{-7} \mathrm{~m}$ reveals that

$$
\Delta P=P_{g}-P_{1}=P_{c}=2 \sigma C_{m}
$$




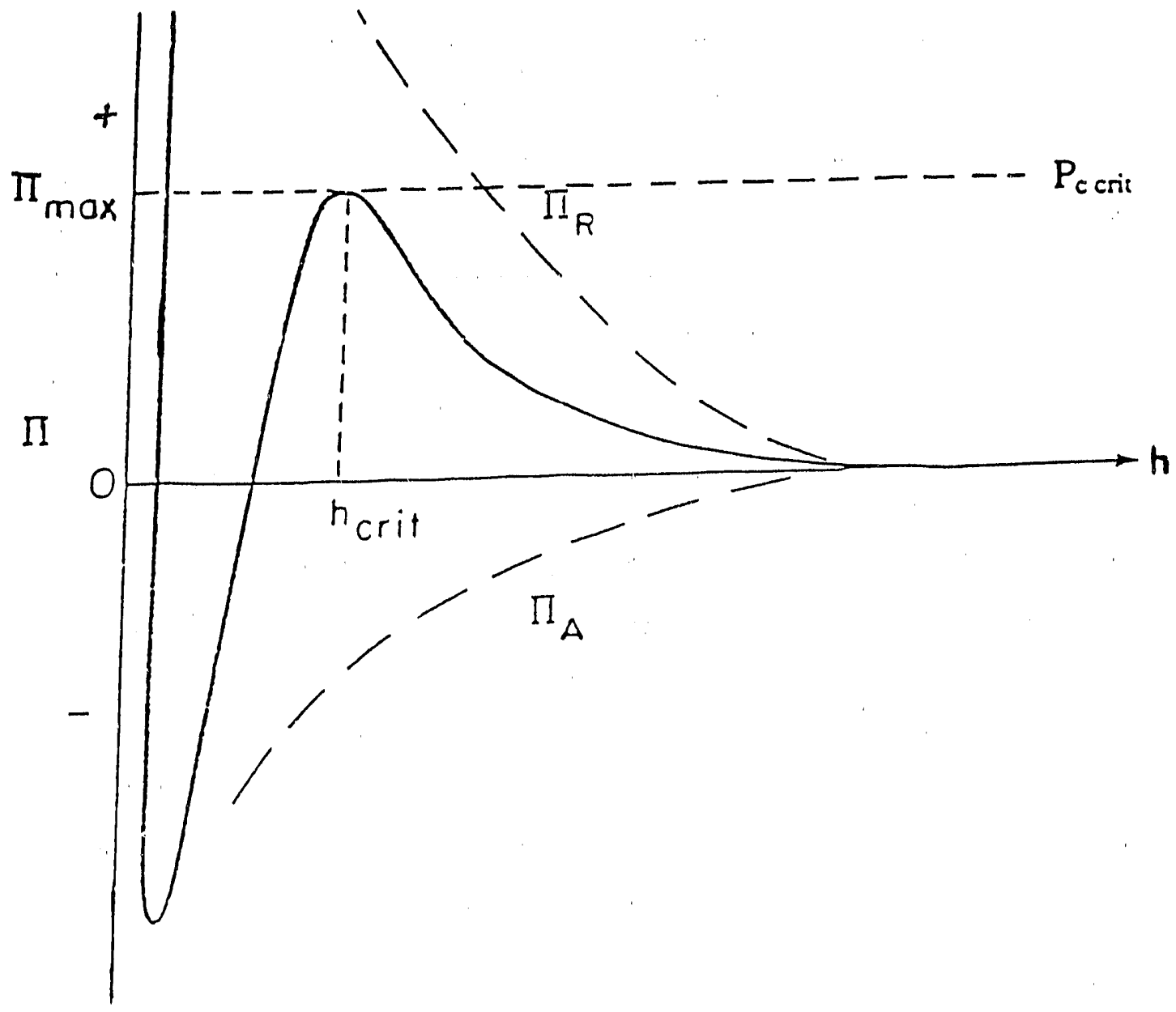

Figure 24: Schematic of a typical disjoining pressure curve. The distances of $h$ are of order 10 to $100 \mathrm{~nm}$, while the values of $\Pi$ are near $10 \mathrm{kPa}$. 


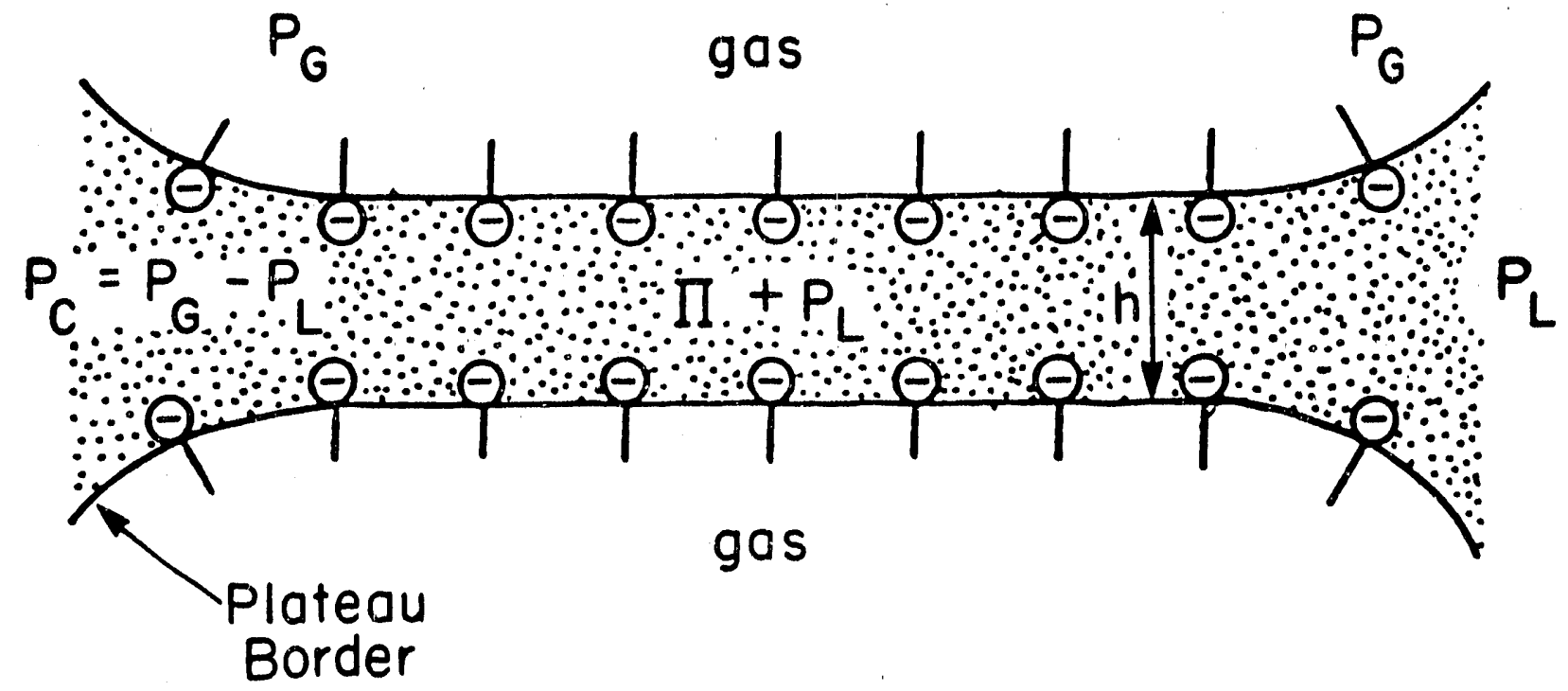

Figure 25: Schematic of a foam lamella. 
where $P_{g}, P_{l}$, and $P_{c}$ are the gas, liquid, and capillary pressures, respectively. Application of Eqn. ( 7$)$ to the center of the lamella in Figure 2.25 where the mean curvature is zero indicates that:

$$
\Delta \mathrm{P}=\Pi \text {. }
$$

Consequently, comparison of Eqns. ( 8) and ( 9) reveals that the capillary and disjoining pressure are equal in the metastable configuration shown in Figure 25.

Thus, as shown in Figure 25, the disjoining pressure must balance the capillary pressure at equilibrium. In the absence of a disjoining pressure, the liquid pressure at the center of th: lamella would equal the gas pressure since the curvature is zero at this point. Eqn ( 8 ), on the other hand, indicates that the gas-phase pressure is greater than the liquid pressure in the Plateau border region. Consequently, liquid wonuld drain from the center of the lamella toward the Plateau borders eventually resulting in the rupture of the lamella. The role of surfactant in determining foam stability in porous media, therefore, relates to the disjoining-pressure isotherm corresponding to a particular surfactant formulation. Surfactants capable of developing large disjoining pressures will be the most effective in stabilizing foam in porous media because they can withstand larger capillary pressures.

Another consequence of the preceding analysis is that large capillary pressures promote foam breakage. As shown in Figure 24, a capillary pressure $P_{c}$ in the porous medium in excess of of the maximum disjoining pressure $\Pi_{\max }$ corresponding to the minimum film thickness $h_{\text {crit }}$ results in foam breakage because a metastable equilibrium film thickness cannot arise. Manlowe [19] noted the collapse of a foam bank at the inlet of a completely gas-saturated micromodel. The $80 \%$ quality foam, comprised of $0.5 \mathrm{wt} \%$ active $\mathrm{C}_{14-16} \alpha$-olefin sulfonate surfactant in deionized water and air, was pregenerated in an upstream micromodel that was etched with an identical flow pattern. As the foam broke at the inlet to the downstream micromodel, surfactant solution began to imbibe into the smanlest pores which resulted in a reduction in the capillary pressure in this water-wet 
system. Eventually, as the capillary pressure decreased with increasing surfactant solution saturation, foam was able to enter and propagate through the model.

\subsection{Dynamic Capillary-Pressure-Induced Coalescence}

An additional consideration in calculating foam stability in porous media relates to the dynamics of foam lamellae transport. As discussed earlier, micromodel studies indicate that foam transports as trains of gas bubbles separated by liquid lamellae. The lamellae conform to the solid boundaries and appear to intersect them with an apparent contact angle of approximately $90^{\circ}$, which is the equilibrium value for a water-wet system $[56,60]$. As a result, the lamellae squeeze and contract as the foam moves through the irregular flow channels in the porous medium. Jimenez and Radke [63] developed a hydrodynamic model to describe the transport and breakage of a single lamella traveling through a sinusoidal pore. The model uses the Reynolds equation for thin-film drainage to describe the radial pressure differential which drives the requisite volume rearrangement of the lamella as it expands and contracts. The model also assumes that an average capillary pressure in the medium, which is set by the saturation of the wetting phase and the surface tension of the system, provides a driving force either to drain or to fill the lamella in response to variations in the disjoining pressure with film thickness. The hydrodynamic model does not account for the "Marangoni-Gibbs effect" directly, which describes a stabilizing stress in the thin film that results from the surface-tension gradients created as the surface area of the lamella increases during transport through a diverging pore. Insufficient data exists on the dynamics of surfactant transport to newly created surfaces to justify including a dynamic Marangoni-Gibbs effect in the model. The model does account for additional viscous dissipation at the gas-liquid interface due to the presence of surfactants, however, by including a parameter $\alpha_{s}$. When $\alpha_{s}=0$ the lamella exhibits an infinitely viscous interface obeying the no-ship condition, which is the upper limit for calculating the viscous dissipation due to the presence of surfactants. For finite values of 
$\alpha_{s}$ the lamella exhibits less resistance to fluid influx or efflux with a corresponding decrease in the importance of surfactants in enhancing viscous dissipation.

An example of a lamella breaking by this mechanism, recorded while injecting $0.005 \mathrm{wt} . \%$ active surfactant solution and air at a total superficial velocity of $45 \mathrm{~m} /$ day and a gas fractional flow of 0.8 into the event micromodel, appears in Figure 26. Figures $26 \mathrm{a}$ and $\mathrm{b}$ show a lamella emerging from a pore constriction in the right center of the photograph and then expanding as it moves into a much wider pore body. Note that the lamella does conform to the pore walls with an apparent contact angle of approximately $90^{\circ}$ as it traverses the flow channel in this figure.

The lamella breaks in Figure $26 \mathrm{c}$ as the thickness of the film reaches the critical thickness for this particular surfactant formulation.

Although it is difficult to discern as a result of the date and time information appearing in the lower right of the photographs, Figure $26 \mathrm{~d}$ indicates that no nearby lamellae broke in response to the lamella breaking in Figure 26c. Figure 27, recorded earlier in the flow study but at the same point in the model and at the same flow conditions, shows that lamella break at characteristic sites in the medium, and that the event described earlier did not result from an external mechanical perturbation to the system. Figure 27 also indicates more clearly that when a single lamella breaks it does not induce nearby lamellae to break. This behavior for an oil-free system is in contrast to observations of foam in a micromodel study conducted in the presence of oil that showed several nearby lamellae breaking each time a pseudoemulsion film broke in the system [19]. The pseudoemulsion film refers to an oil-lamella-gas film as opposed to the gas-lamella-gas filrns addressed in this tiesis.

Typical results for calculations of lamellae coalescence using the hydrodynamic theory of Jimenez and Radke [63] appear in Figure 28 in the form of the dimensionless capillary pressure in the system, $\widetilde{\mathrm{P}}_{\mathrm{c}}^{*}=\mathrm{P}_{\mathrm{c}}^{*} / \mathrm{B}$, versus the ratio of the pore-body to throat radius, $R_{b} / R_{t}$, and as a function of the modified capillary number $\mathrm{Ca}_{m}$. B is referred to as 


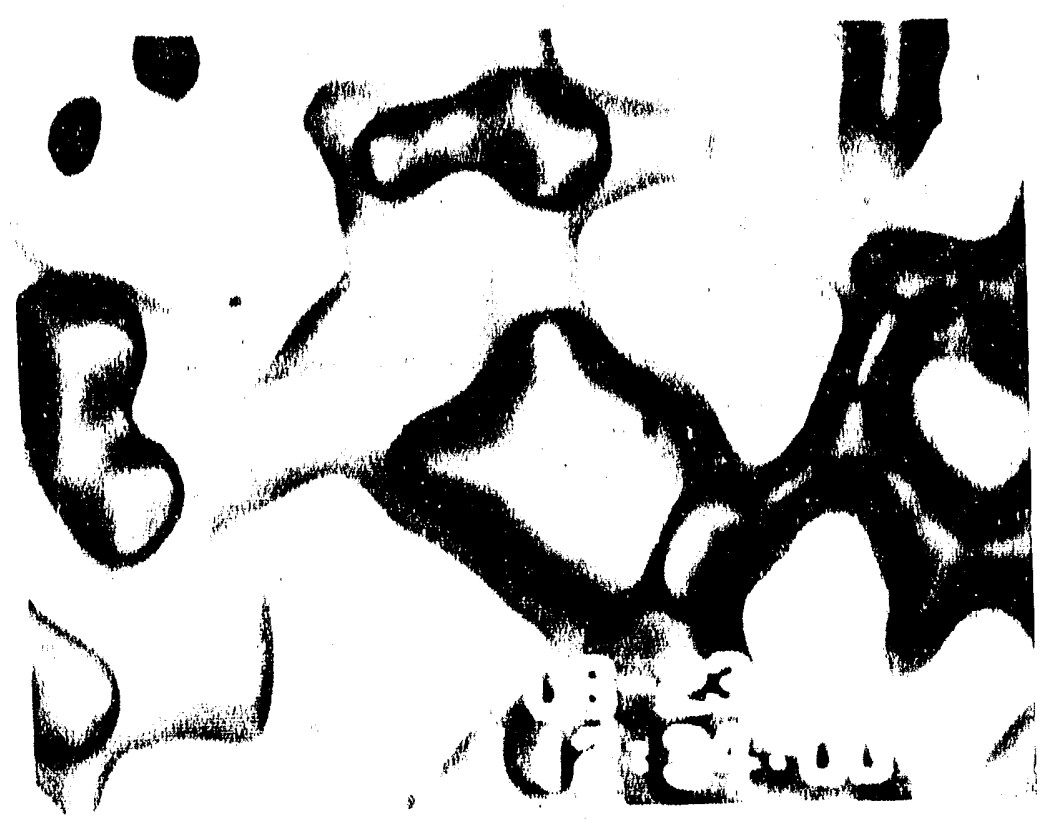

(a)



(b)

Figure 26: Microphotographs of dynamic, capillary-pressure-induced lamella coalescence recorded while injecting an $80 \%$ quality foam comprised of air and surfactant solution at a total superficial velocity of $45 \mathrm{~m} /$ day: (a) lamelia (highlighted with an arrow) convects from right to left into a pore body, (b) lamella stretches as it expands into the pore body because it must conform to the pore walls, 


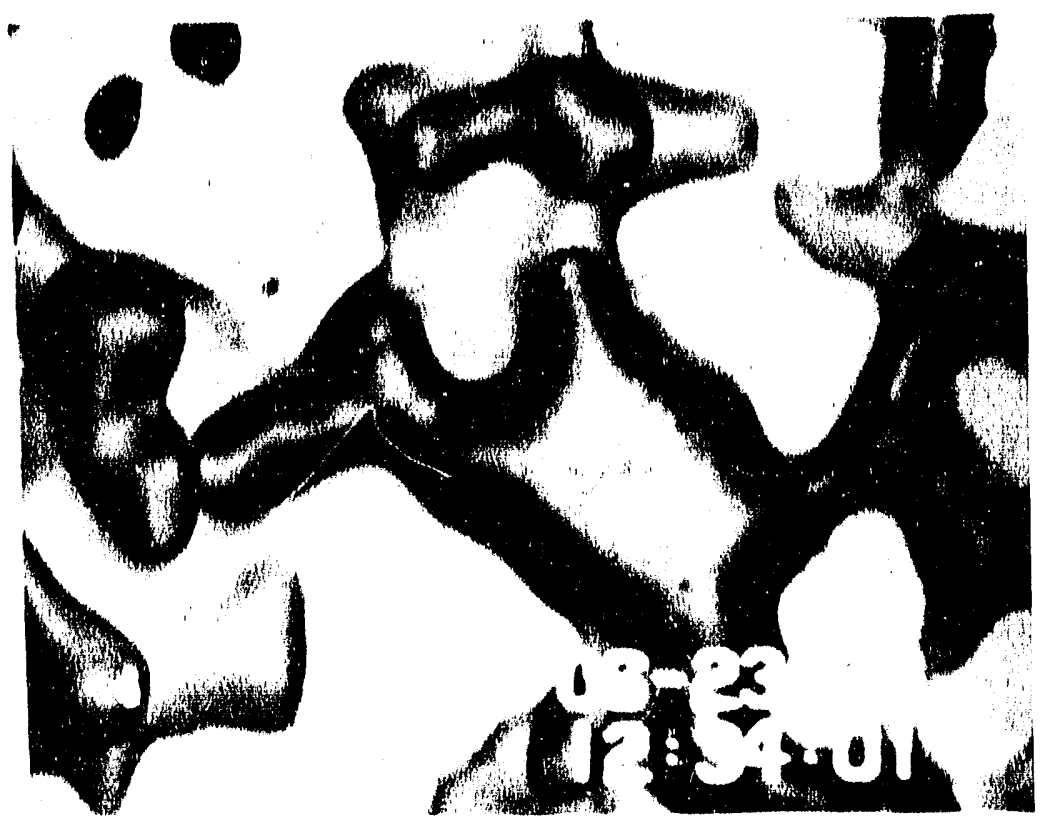

(c)

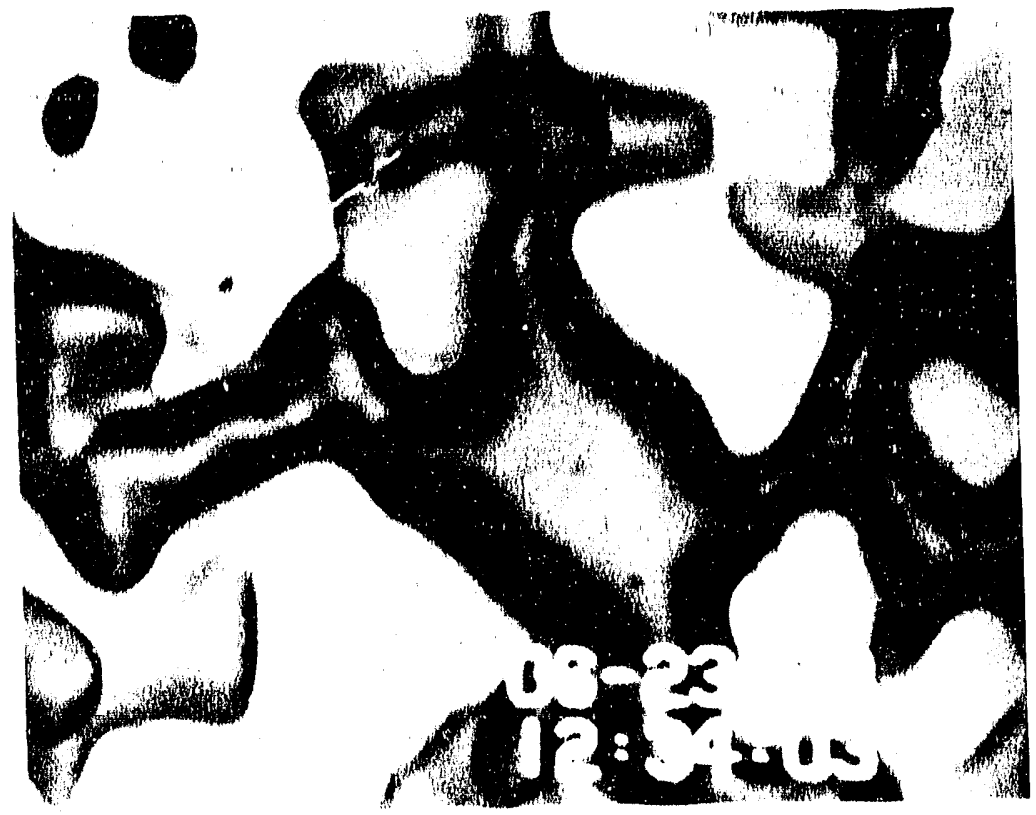

(d)

Figure 26: (c) lamella coalesces at the maximum radius of the pore body, (d) no nearby (cont.) lamellac break in response to the coalescence event in (c). 


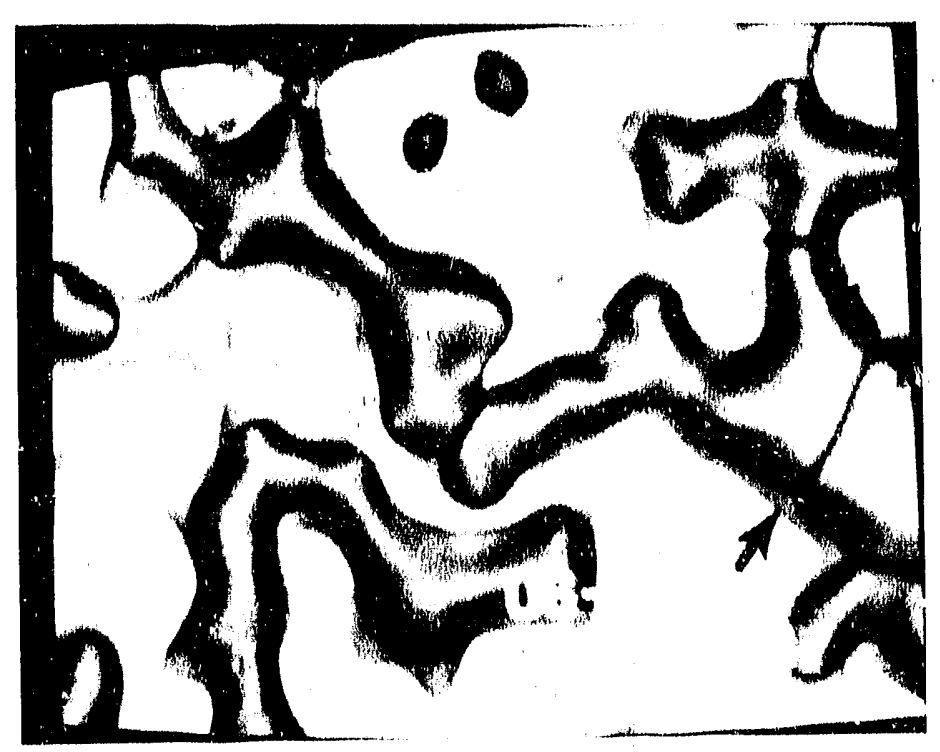

(a)

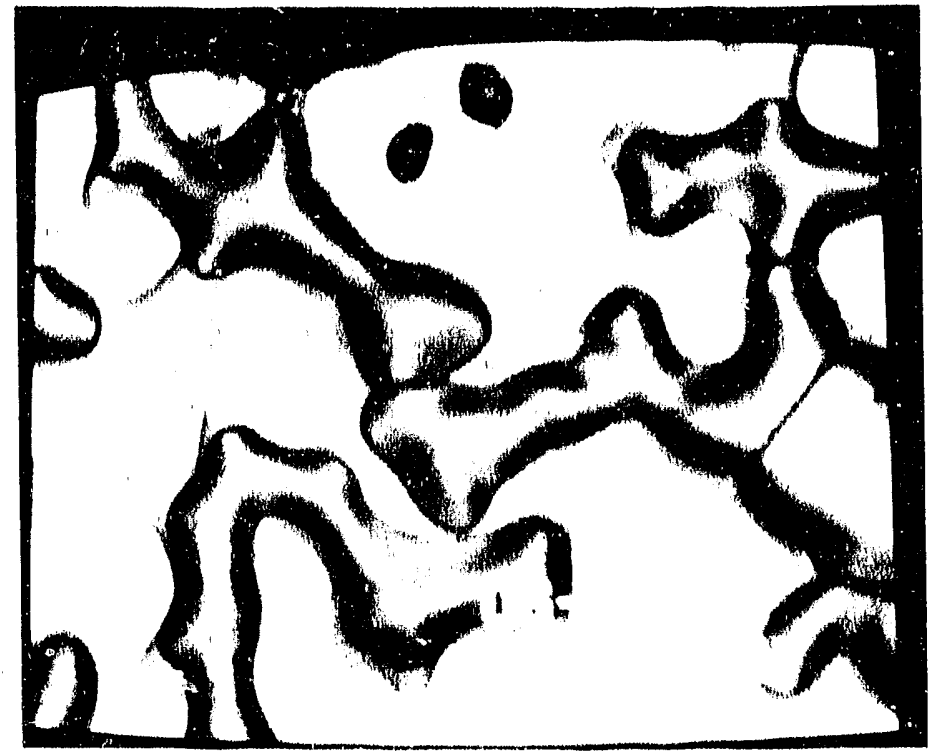

(b)

Figure 27: Microphotographs showing repeatable coalescence at the same site and at the same conditions as Figure 2.26: (a) and (b) lamella conforms to pore walls as it convects through a pore body, 


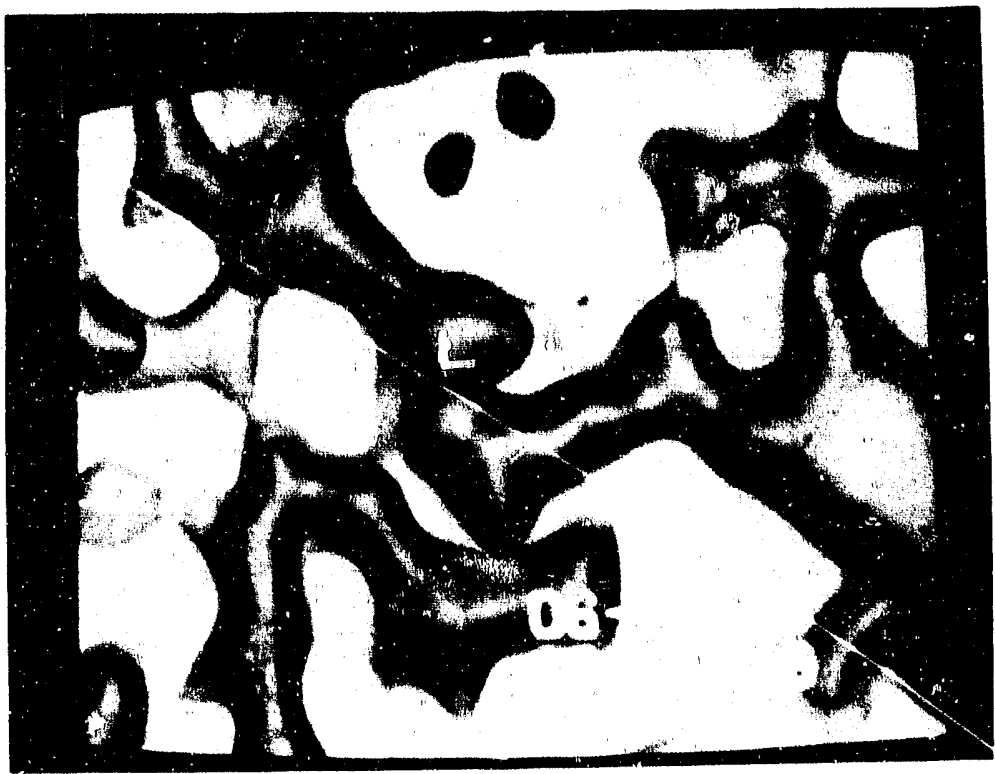

(c)

Figure 27: (c) lamella coalesces without inducing additional coalescence. (cont.) 


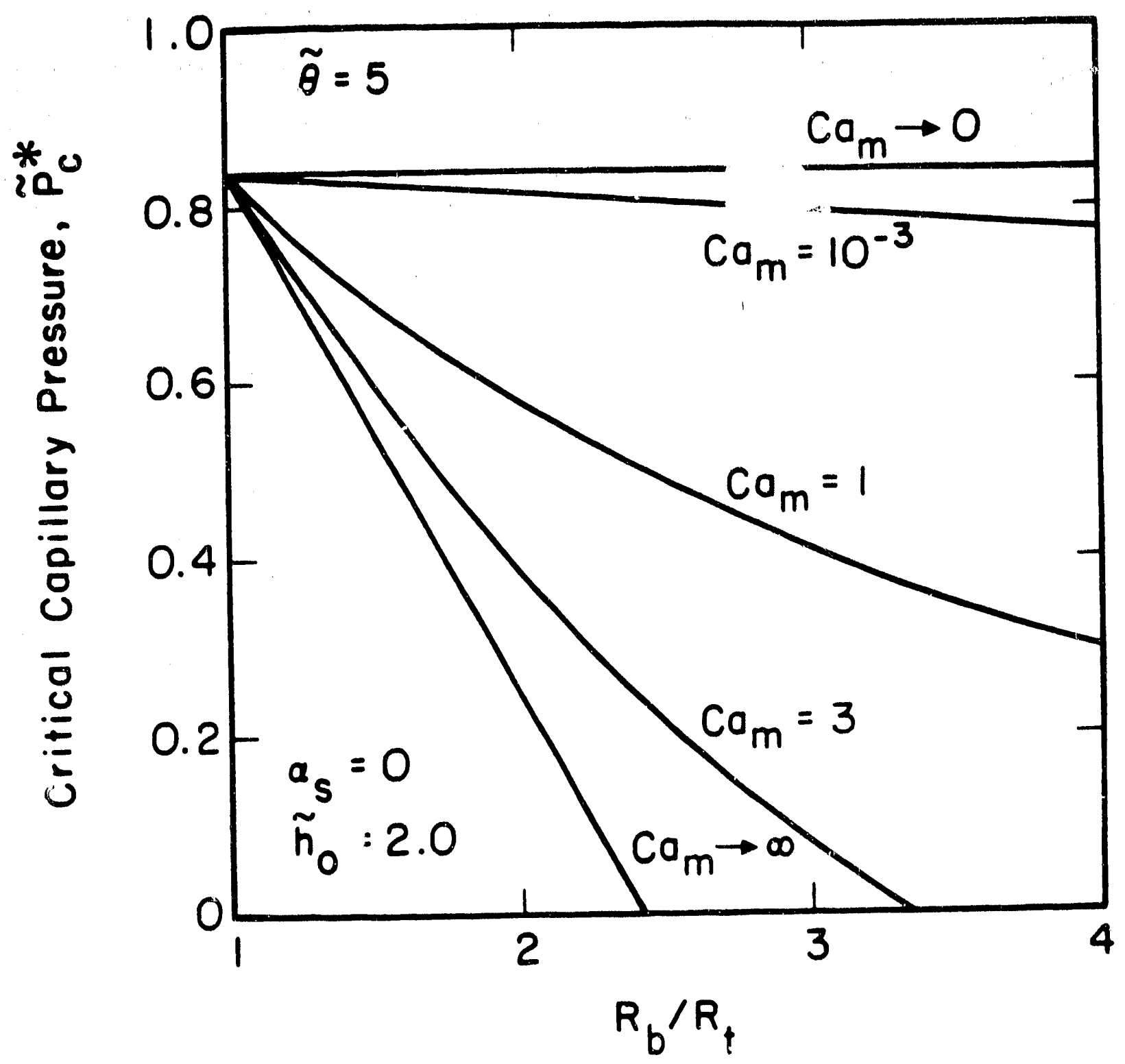

Figure 28: Predictions for critical capillary pressure $\widetilde{P}_{c}{ }^{\circ}$ as a function of the porebody to pore-throat aspect ratio, $R_{b} / R_{t}$, and modified capillary number $C_{m}$ (from Jimenez and Radke [63]). $\mathrm{Ca}_{\mathrm{m}_{\mathrm{i}}}$ is approximately $10^{5}$ times $\mathrm{Ca}$ for the same gas velocity, liquid viscosity, and surface tension. 
the pre-exponential scale factor and it is a known function of the surface potential, ionic strength, surfactant concentration, and temperature $[63,66]$. The modified capiliary number is defined as

$$
\mathrm{Ca}_{\mathrm{m}}=3 \mu_{\mathrm{w}} \mathrm{Q} / 4 \pi \lambda \kappa \mathrm{A}_{\mathrm{H}}
$$

where $\mu_{w}$ is the wetting-liquid viscosity, $Q$ is the gas flow rate through the pore, $\pi$ equals $3.141, \lambda$ is the wavelength of the sinusoidal pore, $1 / K$ is the Debye length (which characterizes the importance of the electric-double-layer-overlap repulsive force), and $A_{H}$ is the Hamaker constant (which characterizes the importance of the van der Waals attractive force). The results appearing in Figure 28 correspond to the constant and low-surfacepotential subcase of the DLVO theory [66]. The factor $\alpha_{s}=0$ appearing in this figure corresponds to a no-slip boundary condition on the gas-liquid interface while $\tilde{\theta}$, which equals $B / \mathrm{K}^{3} \mathrm{~A}_{\mathrm{H}}$, is a ratio of the repulsive to attractive forces within the framework of the DXV theory. The term $\tilde{h}_{0}=2$ corresponds to the dimensionless lamella thickness for initiating the numerical marching routine, which solves for the film profile as a lamella traverses a sinusoidal pore. The results of the calculations are insensitive to this parameter for modified capillary numbers $\mathrm{Ca}_{\mathrm{m}}$ of order one or less. The intersection of a value for the dimensionless capillary pressure $\widetilde{\mathrm{P}}_{\mathrm{c}}{ }^{*}$, corresponding to the saturation and surface tension in the medium, with a value for the modified capillary number $\mathrm{Ca}_{\mathrm{m}}$, based on the parameters in Eqn. (2.10), determines a critical pore-body-to-throat ratio $\widetilde{\mathrm{C}}$. Any bodythroat site in the medium with a ratio larger than this critical value will break lamellae as they convect past. For the same interstitial gas velocity and liquid viscosity, the value of the modified capillary number $\mathrm{Ca}_{\mathrm{m}}$ is roughly $10^{5}$ times that calculated for the more conventional detinition of of the capiiiary number $\widehat{C} a$.

Consequently, the relatively-strong dependence of the critical body-throat ratio shown in Figure 28 for a modified capillary number of order one should apply to realistic flow 
conditions in porous media where interstitial velocities, estimated to be a few feet per day, correspond to capillary numbers of order $10^{-5}$.

The theory presented in Figure 28 predicts increasing lamellae coalescence with increasing capillary number, i.e., increasing gas velocity, at a fixed capillary pressure because the critical value for the ratio of the body-to-throat radius decreases. A larger proportion of the body-throat sites in the medium, therefore, act as "termination" sites in analogy with the "germination" sites for foam generation [54]. Khatib et al. [62] actually measured capillary pressures existing in sand and beadpacks during foam flow experiments. They found that increasing the gas velocity at constant liquid velocity did break pregenerated foam lamellae. Jimenez and Radke [63] were able to match their theory for lamellae coalescence to the experimental results of Khatib et al. [62] for foam flow through an $81-\mu \mathrm{m}^{2}$ sandpack. The match between the theory and the experimental data appears in Figure 29 where the critical dimensionless capillary pressure $\widetilde{P}_{c}^{*}$ is plotted as a function of the dimensionless velocity in the form of the modified capillary number $\mathrm{Ca}_{\mathrm{m}}$. Both the theory and the data clearly show the decrease in the critical capillary pressure with increasing foam velocity. Ir. contrast to the results of Figure 28, the theory presented in Figure 29 is based on the constant-charge case of the DLVO theory $[63,66]$. As a result, the critical capillary pressure has new been made dimensionless by multiplying it by the bulk permittivity of the surfactant solution, $\varepsilon$, and then dividing it $b$, square of the lamella surface charge density q. The factor $\tilde{\theta}$ appearing in the figure, although stili a ratio of the repulsive to attractive forces, is now defined as

$$
\widetilde{\theta}=\frac{2 \pi q^{2}}{\varepsilon \kappa^{3} A_{H}} \text {. }
$$

Another result from the theory presented in Figure 28 is that capillary-pressureinduced lamella coalescence will only occur for lamellae actually flowing in the porous medium. This is true because the capillary pressure, which is set by the saturation of the 




Figure 29: Comparison of experimental and theoretical results for the critical capillary pressure $\widetilde{\mathrm{P}}_{\mathrm{c}}{ }^{\prime}$ as a function of the modified capillary number $\mathrm{Ca}_{\mathrm{m}}$ for an assumed $R_{b} / R_{t}$ of 1.45 for foam coalescence in an $81-\mathrm{mm}^{2}$ sandpack (from Jimenez and Radke [63]). 
wetting phas and the surface tension, acts on all of the lamellae in the medium. If the capillary prossite were high enough to "kill" stationary lamellae, then, according to the theory presented in Figure 28, it would also "kill" all of the flowing lamellae and the entire foam would collapse.

\subsection{Lamella Coalescence by Gas Diffusion}

Another important lamellae coalescence mechanism is gas diffusion driven by the distribution of foam bubble sizes in porous media $[1,11,18,67-70]$. The mechanism of interbubble mass transfer leading to lamellae coalescence is important when a polydisperse bulk foam exists in a large pore body. Differences in curvatures between adjoining gas bubbles exist in such a foam. The Young-Laplace equation relates the pressure drop across a curved interface to the curvature of the interface. The differences in curvatures between adjoining gas bubbles results in a driving force for mass transfer by gas diffusion from the smaller bubbles (higher curvatures) to the larger bubbles (lower curvatures). The magnitude of the differences in curvatures and subsequently the rate of gas diffusion will not be as high in polyederschaum foram, which consists of bubbles (cells) that are nearly polyhedral in shape, compared to kugelschaum foam, which consists of spherical bubbles widely separated from each other by liquid. Foam in porous media will undergo a transition from kugelschaum to polyederschaum as the foam bubbles are squeezed more tightly together with an increase in the capillary pressure. The capillary pressure rises ir response to an increase in the gas (foam) saturation with time during the drainage mode of iujection up to an eventual steady-state value for the gas saturation.

Âlithough a wicie variation in curvatures beiween adjoining gas bubbies áoes noi exist in polyederschaum foam, slight variations in curvatures can still exist when large bubbles are in contact with much smaller bubbles. Figure $30 \mathrm{a}$ illustrates such a 




(a)



(b)

Figure 30: Microphotographs of lamella coalescence in bulk foam due to gas diffusion: (a) two small nearly spherical bubbles (highlighted with an arrow) in contact with iarger foam bubibs, (u) buibtibs shrink due to gas diffusion into larger bubbles, 




(c)

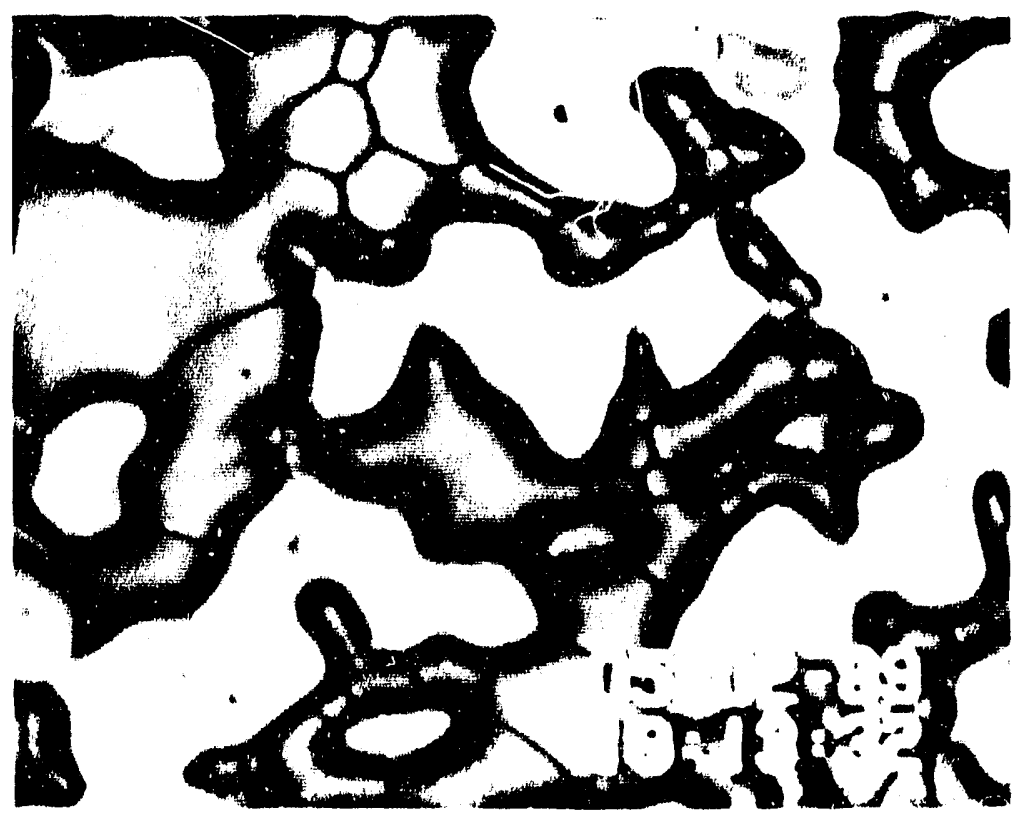

(d)

Figure 30: (c) smal! buthbles continue to shrink at an accelerating pace, (d) a lamella (cont.) coalesces due to the disappearance of the small bubbles. 
configuration where two small, nearly spherical gas bubbles are in contact with several much larger bubbles in a large pore body located at the upper left of the microphotograph. The two small gas bubbles shrink in size over the next several minutes due to gas diffusion, as shown in Figures $30 \mathrm{~b}$ and $\mathrm{c}$. The lamella located between the two small bubbles coalesces as the bubbles finally disappear in Figure 30d. A calculation of the characteristic time for gas diffusion and subsequent collapse of a foam bubble appears in Appendix B. An estimate of a diffusion time for the small bubbles in Figure 30 on the order of 76 seconds results when inputting reasonable values for the fluid properties existing in the micromodel during a foam flood into the characteristic time for diffusion. Among the important properties appearing in the characteristic time are the pressure, temperature, surface tension, diffusion and solubility coefficients for gas within the larnella, lamella thickness, and original bubble radius. The $\mathrm{r}^{\prime}$ asonable agreement between the calculated and observed times substantiates the scaling presented in Appendix B.

Another important characteristic of gas diffusion in bulk foam, first quantified by Monsalve and Schechter [69], is that the initial bubble-size distribution existing in the foam has a profound impact on its subsequent rate of collapse. The importance of the bubblesize distribution can be appreciated by realizing that the shape of the distribution is a measure of the polydispersity of the foam. Narrow distributions correspond to almost uniform bubble sizes and a small driving force for gas diffusion while wide distributions correspond to large variations in bubble sizes and a large driving force for gas diffusion.

Gas diffusion also operates in the lamellae foam regime, but here it will not necessarily coalesce lamellae. Rather lamellae can move along the converging and diverging pore walls in response to pressure gradients leading to gas diffusion until they reach configurations where a driving force for gas diffusion no longer exists. Consequently, the distribution of bubble sizes will change but the number of lamellae will not. 
Figure 31 illustrates this process schematically for a distribution of lamellae in a series of converging-diverging cylindrical pores. The two lamellae located to the left and right of point 1 do not undergo mass transfer because no curvature difference exists between the foam bubbles located on either side of them. The lamella located at point 1 , on the other hand, has a driving force (pressure) for gas diffusion of $4 \sigma / R_{h}(z)$ where $R_{h}(z)$ is the radius of the hemispherical cap corresponding to a particular axial position z. As gas diffuses from left to right across this lamella, its curvature decreases. The lamella must then retreat toward the pore throat, assuming a constant liquid pressure in the Plateau borders, because any curvature less than the hemispherical cap is unstable. The lamella eventually retreats into the pore throat, as shown in Figure $31 \mathrm{~b}$, where no driving force for gas diffusion exists. The process described above is actually too simplistic because it ignores the effect of changes in gas pressure on the lamellae positioned in pore throats to the left and right of point 1 and the subsequent movement of these lamellae. Given sufficient time, however, the configuration illustrated in Figure $31 \mathrm{~b}$ results.

\section{Summary}

Micron dels are valuable tools in uncovering capillary phenomena responsible for lamellae gencration and coalescence during foam fiow in porous media. Among the important generation mechanisms observed are snap-off, weeping-flow breakup, and lamella division and leave behind. Important coalescence mechanisms include dynamic capillary-pressure-induced lamella drainage and gas diffusion. These capillary phenomera are sensitive to the mode of injection, the local capillary environment, and the geometry of the pore structure. For example, weeping-flow breakup of long gas fingers is only important while injecting surfactant solution into a micromodel previously foam-flooded. Likewise, snap-off is sensitive to local variations in the capillary pressure, and it always occurs at the junction of a porc throat and a pore body. The poure-level capiliary pinenumena 


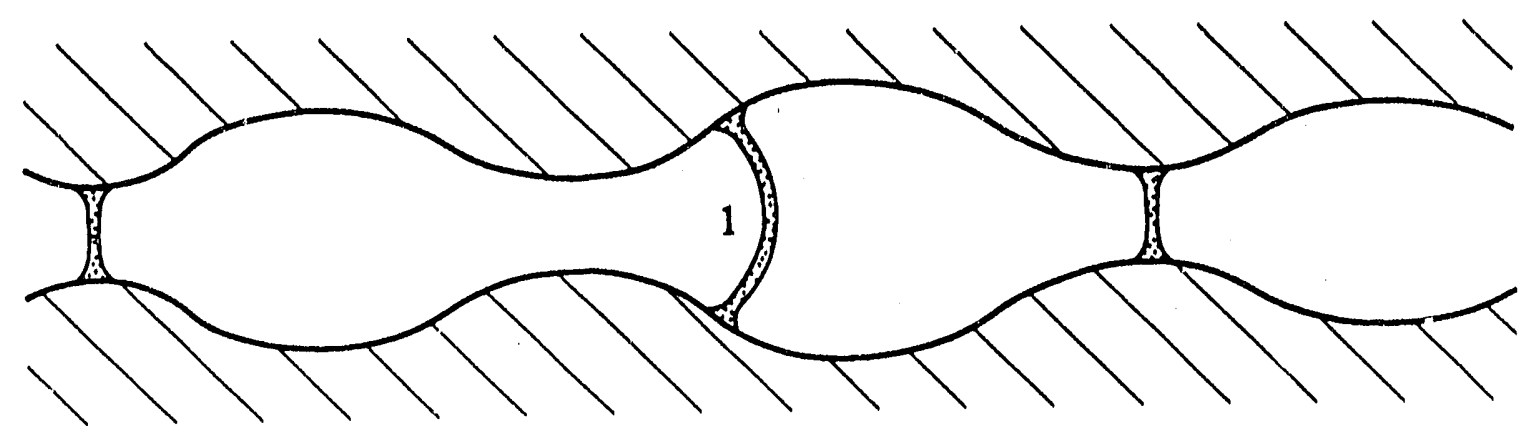

(a) gas diffusion

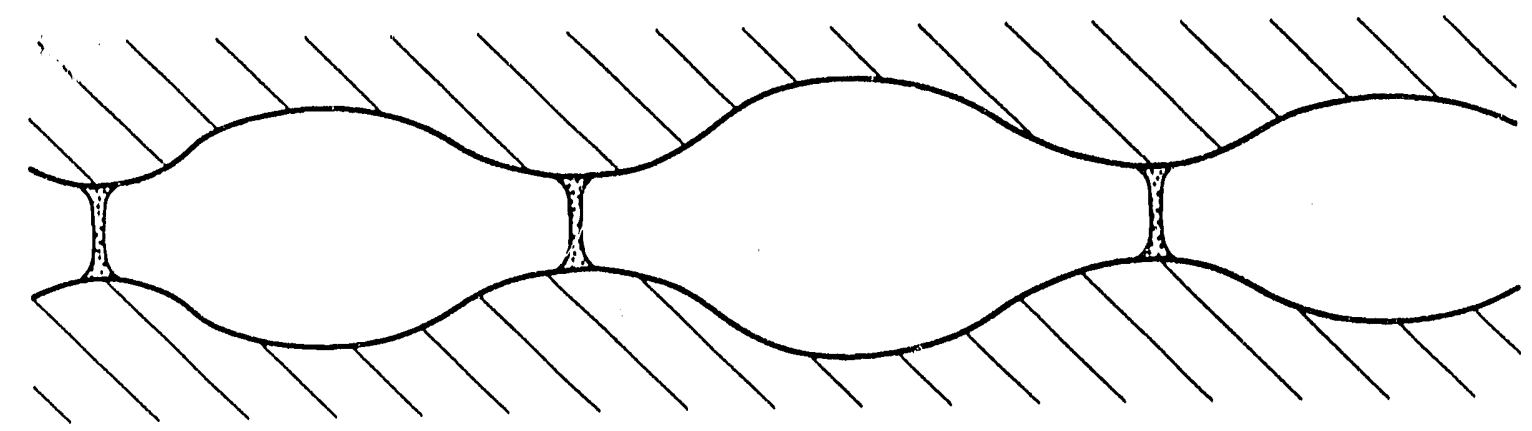

(b) no gas diffusion

Figuite 31: Schematic of gas diffusion without coalescence in lamellae foam: (a) gas diffuses from left to right across a lamella located at point 1 , (b) a stable configuration results without any further driving force for gas diffusion. 
and flow behavior uncovered during this micromodel study serve as the foundation for the mathematical model of foam flow which is developed in the next three chapters.

An important consideration in presenting a tractable model of foam flow behavior is the ability to identify the pore-level mechanisms having the greatest impact on foam texture. The predominant mechanisms will vary depending upon the application for foam as an enhanced oil recovery (EOR) fluid. Both simultaneous gas and surfactant injection and surfactant alternating with gas injection (SAG) have been used to create foam for mobility control in EOR projects. The model developed in the next three chapters is based on simultaneous gas and sırfactant injection during steady-state conditions into a Berea sandstone core. The lamellae generation and coalescence mechanisms included in this model are snap-off, lamella division, and dynamic capillary-pressure-induced lamella drainage. This simplified steady-state model serves as a foundation for developing more complete rate expressions and for extending the population balance to handle transient foam flow behavior. 


\section{Nomenclature}

$$
\begin{aligned}
& A_{H} \quad=\text { Hamaker constant }(J) \\
& \text { B = pre-exponential scale factor for the electrostatic disjoining pressure for } \\
& \text { constant and small degrees of overlap }(\mathrm{Pa}) \\
& \widetilde{\mathrm{C}} \quad=\text { ratio of pore-body to pore-throat radius } \\
& \mathrm{Ca}=\mu_{w} v / \sigma \text {, capillary number } \\
& \mathrm{Ca}_{\mathrm{m}}=3 \mu_{w} \mathrm{Q} / 4 \pi \lambda \kappa A_{H}, \text { modified capillary number } \\
& \mathrm{C}_{\mathrm{m}} \quad=\text { mean curvature }\left(\mathrm{m}^{-1}\right) \\
& \widetilde{\mathrm{C}}_{\mathrm{m}} \quad=\text { dimensionless equilibrium curvature } \\
& \mathrm{C}_{\mathrm{mb}} \quad=\text { curvature in pore body }\left(\mathrm{m}^{-1}\right) \\
& \mathrm{C}_{\mathrm{mt}}{ }^{*} \quad=\text { critical curvature in pore throat }\left(\mathrm{m}^{-1}\right) \\
& \mathrm{d}_{\mathrm{e}} \quad=\text { effective diameter for a noncircular capillary (m) } \\
& \mathrm{F}(\mathrm{e}) \quad=\text { dimensionless function for calculating entry capillary pressure in a noncircular } \\
& \text { capillary } \\
& \mathrm{h}=\text { film thickness }(\mathrm{m}) \\
& h_{c r i t} \quad=\text { critical film thickness for lamella coalescence }(\mathrm{m}) \\
& h_{0} \quad=\text { initial wetting-film thickness }(m) \\
& \tilde{\mathrm{h}}_{\mathrm{o}} \quad=2 \mathrm{kh} \text {, dimensionless film thickness for initiating marching routine for lamella } \\
& \text { coalescence calculations } \\
& \mathrm{p} \quad=\text { fraction of bonds occupied } \\
& \mathrm{p}_{\mathrm{c}} \quad=\text { percolation threshold } \\
& \mathrm{P}_{\mathrm{C}} \quad=\text { capillary pressure }(\mathrm{Pa}) \\
& \mathrm{P}_{\mathrm{c}}^{*} \quad=\text { critical capillary pressure for coalescence }(\mathrm{Pa}) \\
& \widetilde{\mathrm{P}}_{\mathrm{c}}{ }^{*} \quad=\text { dimensionless critical capillary pressure for coalescence } \\
& \mathrm{P}_{\mathrm{g}} \quad=\text { gas pressure }(\mathrm{Pa}) \\
& \mathrm{p}_{1} \quad=\text { liqunid pressure }(\mathrm{Pa})
\end{aligned}
$$




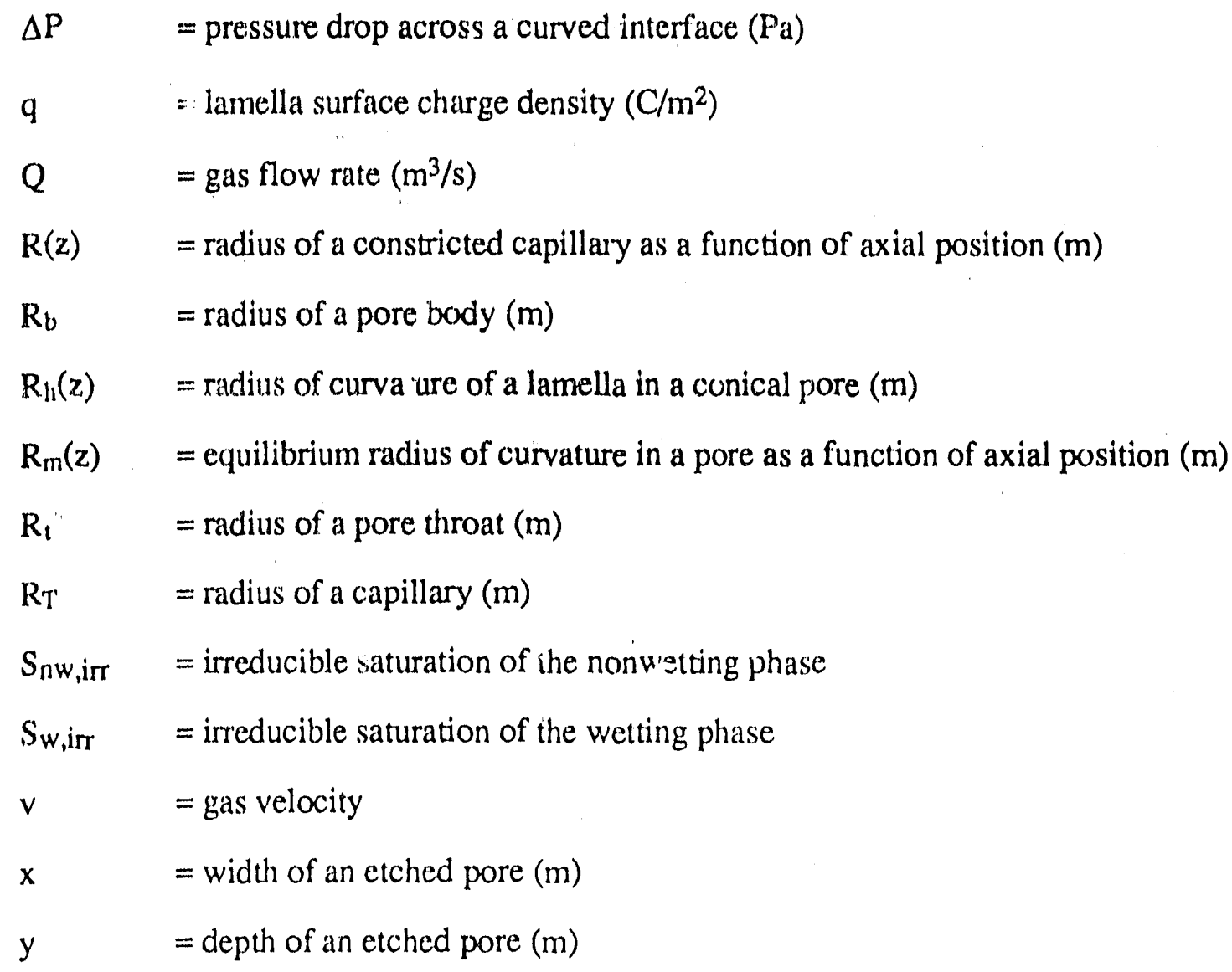

\section{Greek Symbols}

$$
\begin{aligned}
& \alpha_{s} \quad=6 \mu_{w} \kappa R_{t}^{2} / 5 \mu_{s} \text {, surface-viscosity parameter } \\
& \varepsilon=\text { bulk permittirity of the surfactant solution }(\mathrm{F} / \mathrm{m}) \\
& \kappa \quad=\text { inverse Debye length }\left(\mathrm{m}^{-1}\right) \\
& \lambda \quad=\text { wavelength of periodic pore }(\mathrm{m}) \\
& \mu_{\mathrm{s}} \quad=\text { Newtonian surface viscosity }(\mathrm{g} / \mathrm{s}) \\
& \mu_{w} \quad=\text { viscosity of wetling phase }\left(\mathrm{Pa}^{\circ} \cdot \mathrm{s}\right) \\
& \pi \quad=3.141 \ldots . \\
& \Pi \quad=\text { conjoining/disjoining pressure }(\mathrm{Pa}) \\
& \Pi_{\mathrm{A}} \quad=\text { attractive contribution in conjoining/disjoining pressure }\left(\mathrm{P}_{\uparrow}\right)
\end{aligned}
$$


$\Pi_{\max } \quad=$ maximum value in conjoining/disjoining pressure isotherm $(\mathrm{Pa})$

$\Pi_{R} \quad=$ repulsive contribution in conjoining/disjoining pressure isotherm $(\mathrm{Pa})$

$\sigma \quad=$ equilibrium surface tension $(\mathrm{N} / \mathrm{m})$

$\theta \quad=$ contact angle $\left({ }^{\circ}\right)$

$\tilde{\theta}=$ dimensionless ratio of the electrostatic repulsive to Hamaker attractive forces in the conjoining/disjoining pressure isothern, defined in Figure 28 and Eqn. ( 11) 


\section{References}

1. Patzek, T.W.: "Description of Foam Flow in Porous Media by the Population Balance Method," Surfaciant-Based Mobility Control, Smith, D.H., ed., ACS Symposium Series 373 (1988) 326 341.

2. Persoff, P., Radke, C.J., Pruess, K., Benson, S.M., and Witherspoon, P.A.: "A Laboratory Investigation of Foam Flow in Sandstone at Elevated Pressure," paper SPE 18781 presented at the 1989 SPE California Regional Meeting, Bakersfield, CA (April 5-7, 1989) to appear SPERE.

3. Friedmann, F., Chen, W.H., and Gauglitz, P.A.: "Experimental and Simulation Study of High-Temperature Foam Displacement in Porous Media," paper SPE/DOE 17357 presented at the 1988 SPE/DOE Enhanced Oil Recovery Symposium, Tulsa, OK (April 17-20, 1988).

4. Prieditis, J. and Flumerfelt, R.W.: "Mobility of Foam in Porous Media," Surfactant-Based Mobility Control, Smith, D.H., ed., ACS Symposium Series 373 (1988) 295-325.

5. Ettinger, R.A.: "Flow Resistance of Foam in Berea Sandstone," M.S. Thesis, Universily of California, Berkeley (1989).

6. Hirasaki, G.J. anci Lawson, J.E.: Mechanism of Foam Flow in Porous Media: Apparent Viscosity in Smooth Capillaries," SPEJ (April 1985) 176-190.

7. Ettinger, R.A. and Radke, C.j.: "The Influence of Texturt on Steady Foam Flow in Berea Sandstone," paper SPE 19688 presented at the 64th Annual Technical Conferenc of the SPE, San Antonio, TX (Oct. 8-11, 1989).

8. Ginley, G.M. and Radke, C.J.: 'The Influence of Soluble Surfactants on the Flow of Long Bubbles Through a Cylindrical Capillary," in Oil-Field Chemistry; Enhanced Recovery and Production Stimulation, J.K. Borchardt and T.F. Yen, eds., ACS Symposium Series 396, American Chemical Society, Washington, DC, Chapter 26, 481-501 (1989).

9. Jo, E.J.: Displacement of Bubbles and Drops in Fine Capillaries," PhD dissertation, University of Houston, Houston (1984).

10. Rossen, W.R.: "Theories of Foam Mobilization Pressure Gradient," paper SPE/DOE 17358 presented at the 1988 SPE/DOE Enhanced Oil Recovery Symposium, Tulsa, OK (April 17-20, 1988).

11. Falls, A.H., Hirasaki, G.J., Patzek, T.W., Gauglitz, P.A., Miller, D.D., and Ratulowski, J.: "Development of a Mechanistic Foam Simulator: The Fopulation Balance and Generation by Snap-Off," SPERE (August 1988) 884-892. 
12. Huh, D.G. and Kovarik, F.S.: The Effect of Microscopic Heterogeneity on $\mathrm{CO}_{2}$ /Foam Mobility: Part 1- A Mechanistic Study," J. Pet. Tech. (1989) 41, No. 8, 87.2-879.

13. Lenormand, R., Zarcone, C., and Sarr, A.: "Mechanisms of the Displacement of One Fluid by Another in a Network of Capillary Ducts," J. Fluid Mech. (1983) 135, 337-353.

14. Yu, L. and Wardlaw, N.C.: "The Influence of Wettability and Critical Pore-Throat Size Ratio on Snap-Off," J. Colloid Interface Sci. (1986) 109, No. 2, 461-472.

15. Yu, L. and Wardlaw, N.C.: "Mechanisms of Nonwetting Phase Trapping during Imbibition at Slow Rates," J. Colloid Interface Sci. (1986) 109, No. 2, 473-486.

16. Owete, O.S. and Brigham, W.E.: "Flow Behavior of Foam: A Porous Micromodel Study," SPERE (August 1987) 315-323.

17. Shirley, A.I.: "Foam Formation in Porous Media, A Microscopic Visual Study," Surfactant-Based Mobility Control, Smith, D.H., ed., ACS Symposium Series 373 (1988) 234-257.

18. Mast, R.F.: "Microscopic Behavior of Foam in Porous Media," paper SPE 3997 presented at the 1972 SPE Annual Meeting, San Antonio, TX (October 8-11, 1972).

19. Manlowe, D.J.: "Pore-Level Mechanisms of Foam Destabilization by Oil in Porous Media," M.S. Thesis, University of California, Ferkeley (1988).

20. Shirley, A.I.: Personal Communication (1988).

21. Schulman, F. and Zisman, W.A.: "Spreading of Liquids on Low-Energy Surfaces. V. Perfluorodecanoic Acid Monolayers," J. Colloid Interface Sci. (1952) 7, 465-481.

22. Morrow, N.R.: "The Effects of Surface Roughness on Contact Angle with Special Reference to Petroleum! Recovery,"J. Can. Pet. Tech. (October-December, 1975) 42-53.

23. Wardlaw, N.C. and McKellar, M.: "Mercury Porosimetry and the Interpretation of Pore Geometry in Sedimentary Rocks and Artificial Models," Powder Tec'n. (1981) 29, 127-143.

24. Chandler, R., Koplik, J., Lerman, K., and Willemsen, J.F.: "Capillary Displacement and Percolation in Porous Media," J. Fluid Mech. (1982) 119, 249 267.

25. Chatzis, I. and Dullien, F.A.L.: "Modeling Pore Structure by 2-D and 3-D Networks with Application to Sandstones," J. Can. Pet. Tech. (Jan.-March 1977) 97-108.

26. Broadbent, $S:$ and Hammersley, J.M.: "Percolation Processes I Crystals and Mazes," Pl nhil. Soc. (1957) 53, 629-641. 
27. Larson, R.G., Scriven, L.E., and Davis, H.T.: "Percolation Theory of Two-Phase Flow in Porous Media," Chem. Eng. Sci. (1981) 36, 57-73.

28. Larson, R.G. and Morrow, N.R.: "Effects of Sample Size on Capillary Pressures in Porous Media," Powder Tech. (1981) 30, 123-138.

29. Wilkinson, D. and Willemsen, J.F.: "Invasion Percolation: A New Form of Percolation Theory," J. Physics A: Math (1983) 16, 3365-3376.

30. Larson, R.G., Daviz, H.T., and Scriven, L.E.: "Displacement of Residual Nonwetting Fluid From Porous Media," Chem. Eng. Sci. (1981) 36, 75-85.

31. Heiba, A.A., Sahimi, M., Scriven, L.E., and Davis, H.T.: "Percolation Theory of Two-Phase Relative Permeability," paper SPE 11015 presented at the 57th Annual Fall Technical Conference, New Orleans, LA (September 26-29, 1982).

32. Kirkpatrick, S.: "Percolation and Conduction," Rev. Mod. Phys. (1973) 45, No. 4, 574-588.

33. Frisch, H.L., Hammersley, J.M., and Walsh, D.J.A.: "Monte Carlo Estimates of Percolation Probabilities for Various Lattices," Physical Review (1962) 126, No. 3, 949-951.

34. Legait, B.: "Laminar Flow of Two Phases Through a Capillary Tube with Variable Square Cross Section," J. Colloid Interface Sci. (1983) 96, No. 1, 28-38.

35. Denn, M.M.: Process Fluid Mechanics, Prentice-Hall Inc., Englewood Cliffs, NJ (1980).

36. Ransohoff, T.C., Gauglitz, P.A., and Radke, C.J.: "Snap-Off of Gas Bubbles in Stisoothly Constricted Noncircular Capillaries," AlChE J. (1987) 33, No. 5, 753755.

37. Wardlaw, N.C.: Personal Communication (1989).

38. Bretherton, F.P.: "The Motion of Long Bubbles in Tubes," J. Fluid Mech. (1961) 10, 166-187.

39. Everett, D.H. and Haynes, J.M.: "Model Studies of Capillary Condensation I. Cylindrical Pore Model with Zero Contact Angle," J. Colloid Interface Sci. (1972) 38, No. 1, 125-137.

40. Monanty, K.K.: "Fluids in Porous Media: Two-Phase Distribution and Flow," $\mathrm{PhD}$ dissertation, University of Minnesota, Minneapolis (1981).

41. Gauglitz, P.A. and Rarke, C.J.: "An Extended Evolution Equation for Liquid Film Breakup in Cylindrical Capillaries," Chem. Eng. Sci. (1987) 43, No. 7, 14571465.

42. Hammond, P.S.: "Nonlinear Adjustment of a Thin Annular Film of Viscous Fluid Surrounding a Thread of Another Within a Circular Cylindrical Pipe," J. Fluid Mech. (1983) 137, 363-384. 
43. Aul, R.W. and Olbricht, W.L.: "Stability of a Thin Annular Film in PressureDriven, Low Reynolds Number Flow Through a Capillary," J. Fluid Mech. (1990) 215, 585-599.

44. Ratulowski, J. and Chang, H.-C.: "Snap Off at Strong Con: trictions: Effect of Pore Geometry," Surfactant-Based Mobility Control, Smith, D.H., ed., ACS Symposium Series 373 (1988) 282-294.

45. Wardlaw, N.C., Li, Y., and Forbes, D.: "Pore-Throat Size Correlation from Capillary Pressure, Curves," Transport in Porous Media (1987) 2, 597-614.

46. Bernard, G.G. and Holm L.W.: "Effect of Foam on Permeability of Porous Media to Gas," Soc. Pet. Eng. J. (September 1964) 267-274.

47. Roof, J.G.: "Snap-off of Oil Droplets in Water-Wet Pores," Soc. Pet. Eng. J. (March 1970) 85-90.

48. Gauglitz, P.A., St. Laurent, C.M., and Radke, C.J.: "An Experimental Investigation of Gas-Bubble Breakup in Constricted Square Capillaries," J. Pet. Tech. (September 1987) 1137-1146.

49. Mayer, R.P. and Stowe, R.A.: "Mercury Porosimetry-Breakthrough Pressure for Penetration Between Packed Spheres," J. Colloid Sci. (1965) 20, 893-911.

50. Gauglitz, P.A. and Radke, C.J.: "The Role of Wettability in the Breakup of Liquid Films Inside Constricted Capillaries," AIChE Symposium Series (1986) 82, No. 252, 50-63.

51. Ransohoff, T.C. and Radke, C.J.: "Weeping Flow Around Nonwetting Bubbles Trapped in Constricted Noncircular Pores," PhysioChemical Hydrodynamics (1987) 8, No. 3, 255-263.

52. Arriola, A., Willhite, G.P., and Green, D.W.: "Trapping of Oil Drops in a Noncircular Pore Throat and Mobilization Upon Contact with a Surfactant," SPEJ (February 1983) 99-114.

53. Stegemeier, G.L.: "Relationship of Trapped Oil Saturation to Petrophysical Properties of Porous Media," paper SPE 4754 presented at the 1974 SPE Improved Oil Recovery Symposium, Tulsa, OK (April 22-24, 1974).

54. Ransohoff, T.C. and Radke, C.J.: "Mechanisms of Foam Generation in GlassBead Packs," SPERE (May 1988) 573-585.

55. Hirasaki, G.J.: "The Steam-foam Process," supplement to SPE 19505, available from SPE Book Order Department, Richardson, TX (1989).

56. Prideitis, J.: "A Pore-Level Investigation of Foam Flow Behavior in Porous Media," PhD dissertation, University of Houston, Houston, TX (1988).

57. Holm, L.W.: "The Mechanism of Gas and Liquid Flow Through Porous Media in the Presence of Foam," SPEJ (December 1968) 359-369. 
58. Gillis J.V.: "Tracer-Detection and Structure of Stationary Lamellae During Foam Flow Through Berea Sandstone," PhD dissertation, University of California, Berkeley (1990).

59. Nahid, B.H.: "N', sn-Darcy Flow of Gas Through Porous Media in the Presence of Surface Active Agents," PhD Dissertation, University of Southern California, Los Angeles (1971).

60. Falls, A.H., Musters, J.J., and Ratulowski, J.: "The Apparent Viscosity of Foams in Homogeneous Beadpacks," SPERE (May 1988) 155-164.

61. Friedmann, F. and Jensen, J.A.: "Some Parameters Influencing the Formation and Propagation of Foams in Porous Media," paper SPE 15087 presented at the 1986 SPE California Regional Meeting, Oakland, CA (April 2-4, 1986).

62. Khatib, Z.I., Hirasaki, G.J., and Falls, A.H.: "Effects of Capillary Pressure on Coalescence and Phase Mobilities in Foams Flowing Through Porous Media," SPERE (March 1988) 919-926.

63. Jimenez, A.I. and Radke, C.J.: "Dynamic Stability of Foam Lamellae Flowing Through a Periodically Constricted Pore," Oil-Field Chemistry: Enhanced Recovery and Production Stimulation, Borchardt, J.K. and Yen, T.F., ed., ACS Symposium Series 396 (1989) 460-479.

64. Ross, S.: "Foams," Kirk-Othmer Encyclopedia of Technology 3rd. edn., M. Grayson, ed., Wiley Interscience, NY, 127-138, (1980).

65. Virij, A.: "Possible Mechanisms for the Spontaneous Rupture of Thin Free Liquid Films," Disc. Faraday Soc. (1966) 42, 23-33.

66. Verwey, E.J.W. and Overbeek, J.T.G.: The Theory of Lyophobic Colloids, Elsevier, Amsterdam (1948).

67. Heller, J.P. and Kuntamukkula, M.S.: "Critical Review of the Foam Rheology Literature," Ind. Eng. Chem. Res. (1987) 26, 318-325.

68. Falls, A.H., Lawson, J.B., and Hirasaki, G.J.: "The Role of Noncondensable Gas in Steam Foams," J. Pet. Tech. (January 1988) 95-104.

69. Monsalve, A. and Schechter, R.S.: "The Stability of Foams: Dependence of Observation on the Bubble Size Distribution," J. Colloid Interface Sci. (1984) 97, No. 2, 327-335.

70. de Vries, A.J.: "A Fundamental Investigation of the Factors Controlling the Stability of Foams," Rec. Trav. Chim. (1958) 77, 81-90. 
A $\quad p \quad p \quad$ e $n$ d $\quad$ i $\quad x$

A detalled drawing of the camera assembly used with the micromodel flow apparatus is shown in fig. A.1. Table A.l provides a listing of the make and model number for the equipment detailed in Fig. A.l. The assembly allowed for maximum flexibility in magnification. The bellows provided multiple focal lengths depending on the necessary magnification. The microscope lens added additional magnification for viewing pore-level events in the micromodel. Note that the entire assembiy was attached to a movable stage. This was necessary to allow for focusing at all possible magnification levels.

Table A.1: Equipment listing for Figure A.l

Video camera: Javelin Electronics: Model JE3040CC C-mount: Nikkor: Model $55 \mathrm{~mm}$ c-mount adapter Bellows: Silge and Kuhne: Model OTHOPHOT 1198 Lens: Ernst Leitz Wetzlar: Model summar $f=8 \mathrm{~cm}$ 
Appendix A cont

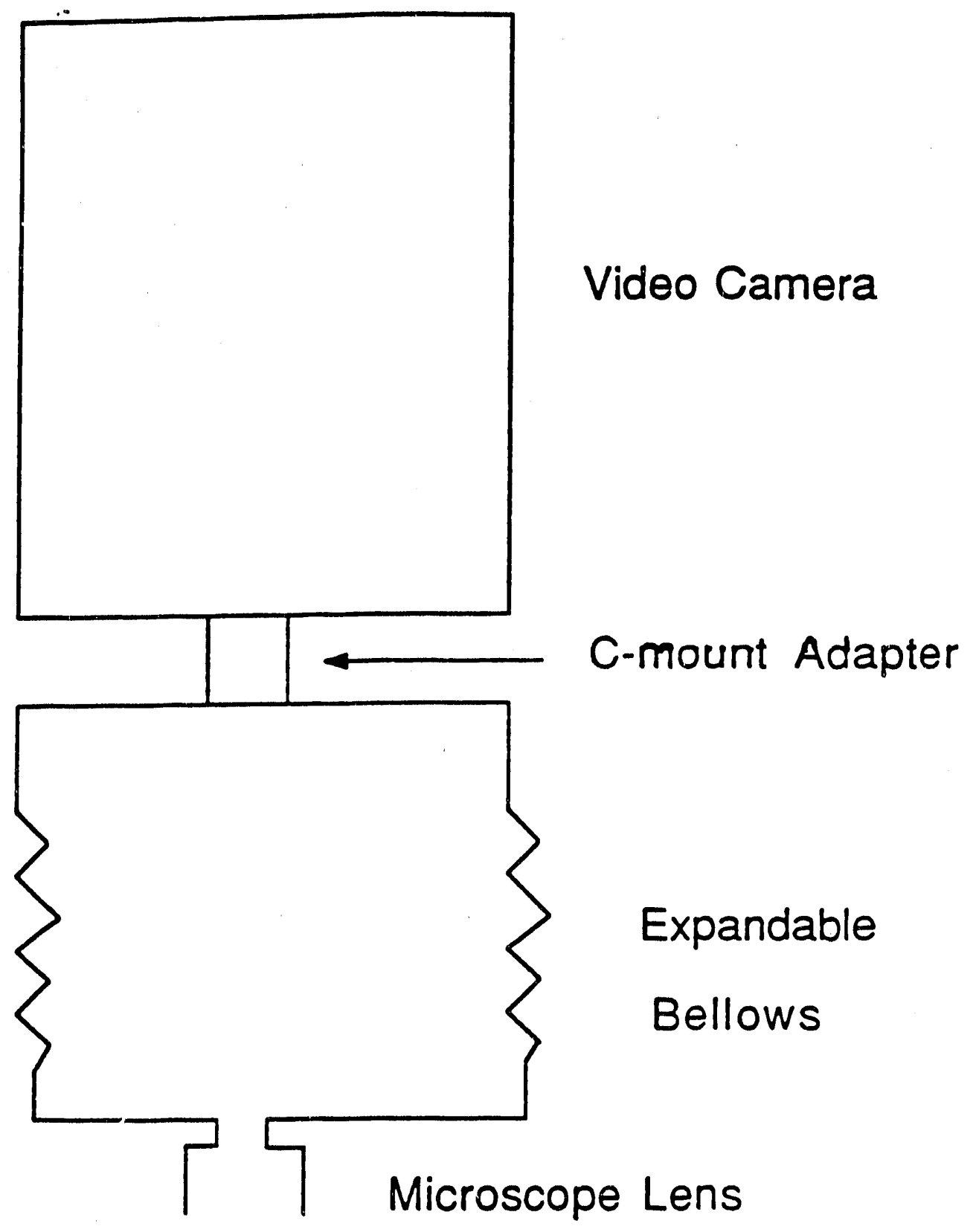

Figure A.1: Schematic of camera assembly used in the foam-flow apparatus. 


\section{Appendix B}

\section{Characteristic Time for Gas Diffusion in Bulk Foam}

Not surprisingly the diffusion equation serves as the foundation for calculating a characteristic time for gas diffusion in bulk foam [1]. The one-dimensional form of this equation may be written for the case of a constant diffusion coefticient $D$ as

$$
-\frac{\partial \mathrm{n}}{\partial \mathrm{t}}=\mathrm{DA} \frac{\partial \mathrm{c}}{\partial \mathrm{x}}
$$

where $\partial \mathrm{n} / \partial \mathrm{t}$ is the number of moles of gas passing per unit time through the film surrounding a gas bubble, $\mathrm{A}$ is the area of the film, and $\partial \mathrm{c} / \partial \mathrm{x}$ is the concentration gradient of gas in the liquid film. The concentration gradient can be approximated as $\Delta \mathrm{c} / \mathrm{h}$ where $\Delta \mathrm{c}$ is the difference in concentration across and $h$ is the thickness of the film. Henry's law states that the concentration difference is proportional to the pressure drop $\Delta \mathrm{P}$ across the film, or:

$$
\Delta c=S \Delta P=S \frac{4 \sigma}{R_{\text {bub }}}
$$

where $S$ is the solubility coefficient of gas in the liquid in moles of gas per unit volume of liquid and per unit area, $\sigma$ is the surface tension, and $R_{\text {bub }}$ is the radius of the film which for all practical purposes is equivalent to the radius of the gas bubble. Eqns. (B1) and (B2) can be combined, assuming a spherical foam bubble, to show that

$$
-\frac{\mathrm{dn}}{\mathrm{dt}}=4 \pi \mathrm{R}_{\text {bub }}^{2} \mathrm{D}\left(\frac{4 \sigma S}{\mathrm{R}_{\text {bub }} h}\right)
$$

The number of moles of gas in the bubble may be expressed as a function of the bubble radius for the case of an ideal gas in the following manner: 


$$
n=\frac{4 \pi R_{\text {bub }}^{3}}{3 R_{g} T}\left(P_{0}+\frac{4 \sigma}{R_{\text {bub }}}\right)
$$

where $R_{g}$ is the universal gas constant, $T$ is the absolute temperature, and $P_{0}$ is the gas pressure outside of the bubble. The excess pressure in the gas bubble, $4 \sigma / R_{\text {bub }}$, is usually much smaller than the external gas pressure and therefore may be neglected in Eqn. (B4). The following expression emerges after inserting Eqn. (B4) into Eqn. (B3) and integrating:

$$
R_{\text {bub }, 0}^{2}-R_{\text {bub }}^{2}=\frac{8 R_{g} \text { TDS } \sigma t}{P_{0} h}
$$

where $R_{\text {bub,o }}$ is the original radius of the bubble. The characteristic time for a bubble to disappear by gas diffusion, $t_{\text {dif }}$, then follows as

$$
t_{d i f}=\frac{R_{b u b, o}{ }^{2} P_{o} h}{8 R_{g} T D S \sigma}
$$

An estimate of 76 seconds emerges for the difiusion time corresponding to the disappearance of the bubbles illustrated in Figure 30 when inputting the following reasonable values for the micromodel sysic $\mathrm{m}: \mathrm{R}_{\text {bub,o }}=1.50 \times 10^{-4} \mathrm{~m} ; \mathrm{P}_{\mathrm{o}}=1.3 \times 10^{5} \mathrm{~Pa}$; $\mathrm{R}_{\mathrm{g}}=8.3 \mathrm{~Pa} \cdot \mathrm{m}^{3} /(\mathrm{mole} \cdot \mathrm{K}) ; \mathrm{T}=298 \mathrm{~K} ; \mathrm{D}=10^{-9} \mathrm{~m}^{2} / \mathrm{s} ; \mathrm{S}=5.0 \times 10^{-6} \mathrm{moles} /\left(\mathrm{m}^{3} \cdot \mathrm{Pa}\right) ; \mathrm{h}=$ $10^{-7} \mathrm{~m}$; and $\sigma=3.9 \times 10^{-2} \mathrm{~N} / \mathrm{m}$. This calculated value compares well with the measured value of approximately 114 seconds.

\section{References}

1. de Vries, A.J.: "A Fundamental Investigation of the Factors Controlling the Stability of Foams," Rec. Trav. Chim. (1958) 77, 81-90. 

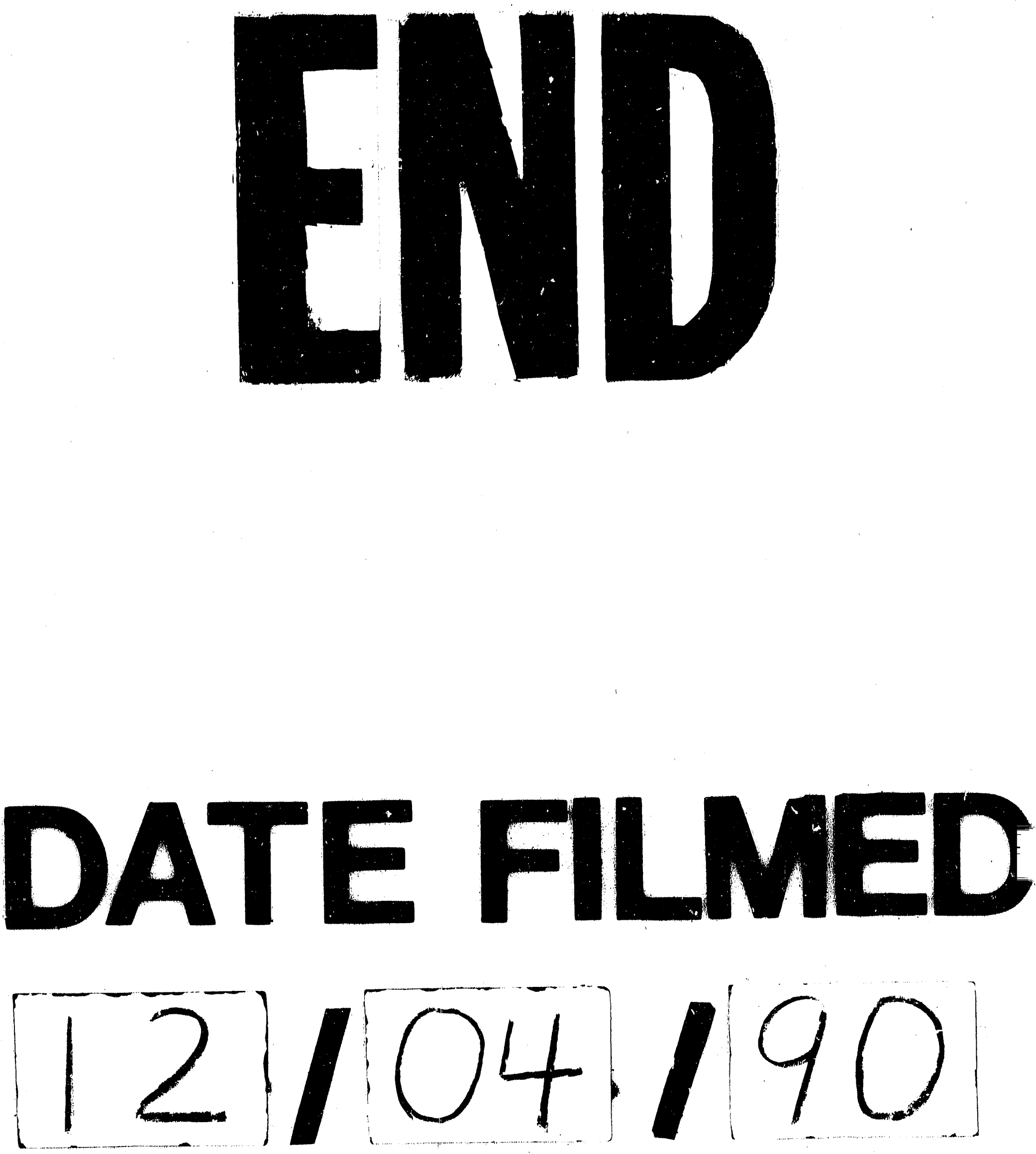
